\title{
Magnetization and elastic dynamics in nanostructured metamaterials
}

\section{Dissertation}

\author{
for the award of the degree \\ "Doctor of Philosophy" Ph.D. Division of \\ Mathematics and Natural Sciences \\ of the Georg-August-Universität Göttingen \\ within the doctoral program PROPHYS \\ of the Georg-August University School of \\ Science
}

Submitted by

Maria Mansurova

from Moscow, Russia

Göttingen, 2016 


\section{Thesis Committee}

Prof. Dr. Markus Münzenberg Institut für Physik, Ernst-Moritz-ArndtUniversität

Prof. Dr. Hans-Ulrich Krebs Institut für Materialphysik, Georg-AugustUniversität

\section{Members of the Examination Board}

Referee: Prof. Dr. Markus Münzenberg, Institut für Physik, Ernst-Moritz-Arndt-Universität

Co-Referee: $\quad$ Prof. Dr. Hans-Ulrich Krebs, Institut für Materialphysik, Georg-August-Universität

Other Members: Prof. Dr. Claus Ropers, IV. Physikalisches Institut, Georg-August-Universität

Prof. Dr. Marcus Müller, Institut für Theoretische Physik, Georg-August-Universität

Prof. Dr. Vasile Mosneaga, I. Physikalisches Institut, Georg-August-Universität

Prof. Dr. Cynthia Volkert, Institut für Materialphysik, Georg-August-Universität

Date of the oral examination: 19.02.2016 


\section{Acknowledgments}

First of all, I am deeply grateful to my supervisor Markus Münzenberg for his constant support and scientific guidance.

I have to acknowledge also the good company and help of all my colleagues both in Göttingen and Greifswald, specially Martin Lüttich, Steffen Wittrock, Christian Denker, Ulrike Martens, Thomas Schumann and Robin John.

- Benjamin Lenk and Fabio Boschini, for first introducing me to working with ultrafast lasers and the magneto-optical setup.

- Jakob Walowski for many discussions, ultrafast laser troubleshooting and practical advices.

- Marvin von der Ehe, Vladyslav Zbarsky and Christian Leutenantsmeyer for their help with CoFeB samples.

- Florian Döring for providing and characterization of multilayer samples.

- Markus Müller and Dennis Meyer for the new insights into elastic dynamics through their simulation software.

- Henning Ulrichs for many fruitful and stimulating discussions.

- Stefan Behrenz, Judith von der Haar and Jelena Panke for asking many questions and good lab work.

At last, I thank my family, friends and Israel for their encouragement and support along the way. 


\section{Abstract}

In this work, magnetization and elastic dynamics in nano-structured artificial materials have been investigated in an all-optical, time-resolved, pump-probe experimental setup. Rich dynamics are brought about by means of the ultrafast laser excitation, that generates a picosecond heat gradient. This induces coherent, dynamic processes that are observed with a second, time-delayed probe pulse. In a two-dimensional magnonic crystal consisting of a sub-micron antidot square lattice on a CoFeB ferromagnetic thin film, spin-wave modes with a weak frequency dependence on the applied magnetic field are found. This suggests spin-wave localization in regions of inhomogeneous internal field. Elastic dynamics on the same structure show frequencies proportional to characteristic lattice lengths (distance between antidots and their size) pointing to the excitation of surface stress waves. On multilayer $\mathrm{CoFeB} / \mathrm{MgO}$ structures, that have matching acoustic impedance, both surface and bulk elastic waves were detected in good qualitative agreement with theory. Elastic dynamics measurements in transmission and reflection geometries show that the amplitude of the high-frequency surface mode can be efficiently suppressed by breaking the periodicity of the multilayer. In addition, in the acoustically mismatched W/PC multilayers the excitation of bulk-mode frequencies is hindered. 


\section{Kurzfassung}

In dieser Arbeit wurde magnetische und elastische Dynamik in nanostrukturierten künstlichen Materialien mit Hilfe eines optischen, zeitaufgelösten Pumpprobe Messaufbaus untersucht. Die Absorption der ultraschnellen Laserpulse erzeugt einen Wärmegradienten auf einer Zeitskala von Pikosekunden. Dieser induziert kohärente dynamische Prozesse, welche mit einem zweiten, zeitverzögerten Puls beobachtet werden. In einem zweidimensionalen magnonischen Kristall, bestehend aus einem submikrometer großen Antidotgitter auf einer ferromagnetischen $\mathrm{CoFeB}$ Schicht, können Spinwellenmoden beobachtet werden, die eine schwache Frequenzabhängigkeit vom externen magnetischen Feld aufweisen. Dies lässt vermuten, dass Spinwellen in der Nähe von Inhomogenitäten des internen Feldes lokalisieren. Elastische Dynamik auf denselben Strukturen zeigt Frequenzen proportional zu charakteristischen Strukturgrößen (Antidotabstand und Antidotgröße), was auf die Anregung von Spannungswellen auf der Oberfläche hindeutet. Auf $\mathrm{CoFeB} / \mathrm{MgO}$ Schichtstapeln mit ähnlicher akustischer Impedanz, können sowohl Oberflächenwellen als auch Wellen im Volumen in guter Übereinstimmung mit der Theorie beobachtet werden. Anregung der elastischen Dynamik in Reflektions- und Transmissionsgeometrie zeigen, dass durch das Brechen der Periodizität des Schichtstapels die Amplitude der hochfrequenten Oberflächenwelle effektiv unterdrückt wird. Außerdem sind im W/PC Schichtstapeln mit hohem akustischem Versatz innere Wellen unterdrückt. 


\section{Contents}

\begin{tabular}{lll}
\hline & Introduction & 1
\end{tabular}

\begin{tabular}{|lll}
\hline 2 & Theoretical considerations & 5
\end{tabular}

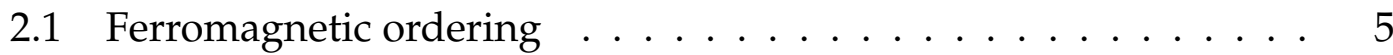

2.1.1 Static magnetization properties . . . . . . . . . . . 7

2.1 .2 Spin waves in bulk materials $\ldots \ldots \ldots$. . . . . . . 8

2.1 .3 Spin waves in thin films . . . . . . . . . . . . . . . . 9

$2.1 .4 \quad$ Magnonic crystals $\ldots \ldots \ldots \ldots . \ldots . \ldots 11$

2.2 Elastic dynamics in multilayers . . . . . . . . . . . . . . . . 14

2.3 Excitation mechanism . . . . . . . . . . . . . . . . . . . . . . . . . . . . . . . 18

$2.3 .1 \quad$ Elastic dynamics . . . . . . . . . . . . . . . . . 18

2.3 .2 Magnetization oscillations . . . . . . . . . . . . . . 21

$\begin{array}{lll}3 & \text { Methods } & 23\end{array}$

3.1 Experimental implementation . . . . . . . . . . . . . . . . . 23

3.2 Jones calculus formalism . . . . . . . . . . . . . . . . 25

3.2.1 Reflectivity dynamics detection setup . . . . . . . . . . . . 27

3.2.2 Magneto-optical Kerr effect detection setup . . . . . . . . . 27

3.3 Samples: description and characterization . . . . . . . . . . . 32

3.3.1 Magnonic crystals on thin CoFeB film . . . . . . . . . . . . 33

3.3 .2 Multilayer structures $\ldots \ldots \ldots \ldots$

3.3 .3 Bismuth telluride . . . . . . . . . . . . . . . . . 36

4 Magnetization dynamics in sub-micron antidot lattices on thin CoFeB

films 39

4.1 General spin-wave properties: From continuous CoFeB thin films

to a magnonic crystal . . . . . . . . . . . . . . 40

4.1.1 Interface between a magnonic crystal and continuous film 42

4.1.2 Magnetization dynamics on defect lines in a magnonic

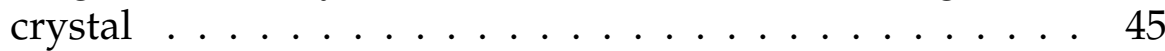

4.1 .3 Discussion . . . . . . . . . . . . . . . . . . . 47 
4.2 Coexistence of perpendicular standing spin wave and DamonEshbach spin waves in a magnonic crystal . . . . . . . . . . . . . 4 48

4.3 Sub-micron magnonic crystals . . . . . . . . . . . . . . . . 52

$4.3 .1 \quad$ Elastic dynamics $\ldots \ldots \ldots \ldots$. . . . . . . . . . . . . . . . . . . . . 52

$4.3 .2 \quad$ Magnetization dynamics . . . . . . . . . . . . . . . . . . 55

4.3 .3 Discussion . . . . . . . . . . . . . . . . . 59

4.4 Heating effects on properties of magnetization oscillation . . . . . 61

5 Elastic dynamics in multilayer structures $\quad 67$

5.1 Introduction: the role of acoustic mismatch . . . . . . . . . . . 67

$5.2 \mathrm{~W} / \mathrm{PC}$ multilayer structures . . . . . . . . . . . . . . . . . 71

5.2.1 Single $\mathrm{W}$ layers: general considerations . . . . . . . . . . 71

$5.2 .2 \mathrm{~W} / \mathrm{PC}$ double layers $\ldots \ldots \ldots \ldots . \ldots 74$

$5.2 .3 \quad \mathrm{~W} / \mathrm{PC}$ multilayers $\ldots \ldots \ldots \ldots$. . . . . . . . 78

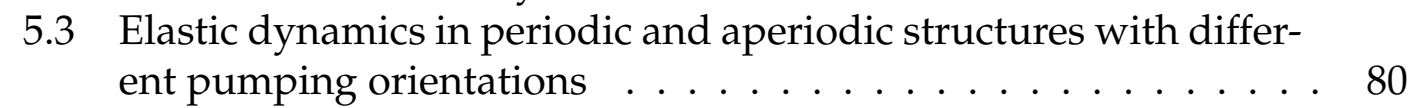

5.4 Discussion $\ldots \ldots \ldots \ldots \ldots \ldots \ldots$

\begin{tabular}{lll}
\hline & Conclusion and outlook & 85
\end{tabular} 


\section{List of Figures}

$2.1 \quad$ Calculated free energy of a CoFeB thin film . . . . . . . . . 8

\begin{tabular}{ll}
2.2 & Calculated band structure of spin wave in an antidot lattice on \\
\hline CoFeB thin film $\ldots \ldots \ldots \ldots \ldots \ldots \ldots \ldots$ & $\ldots \ldots \ldots \ldots \ldots$
\end{tabular}

$2.3 \quad$ A SEM image of an antidot lattice on a CoFeB thin film . . . . . 12

2.4 Calculated reflection coefficient of a slab of material . . . . . . . 16

2.5 Calculated dispersion relation of an elastic wave in an infinite

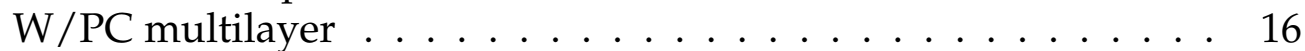

2.6 Calculated dispersion relation of an elastic wave in an infinite $\mathrm{CoFeB} / \mathrm{MgO}$ multilayers $\ldots \ldots \ldots \ldots \ldots \ldots$

3.1 Experimental setup for the detection of magnetization and reflec-

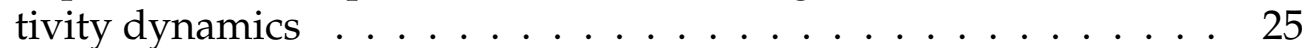

3.2 Definition of the coordinate system and a schematics of polar, longitudinal and transverse MOKE $\ldots \ldots \ldots \ldots \ldots \ldots$

3.3 Saturation magnetization as function of temperature and hysteresis of a $50-n m$ CoFeB film $\ldots \ldots \ldots \ldots$. . . . . . . . . . . .

3.4 SEM images of antidot structures with different periodicities and antidot sizes milled using FIB $\ldots \ldots \ldots \ldots \ldots$. . . . . . . . . 34

3.5 SEM image of antidot waveguides milled on a CoFeB film using

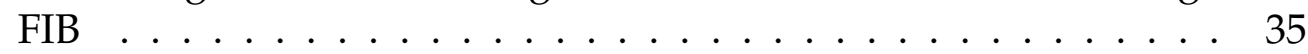

$3.6 \quad$ TEM images of $\mathrm{CoFeB} / \mathrm{MgO}$ multilayers in cross section . . . . . 37

3.7 Relative orientation of $\mathrm{Bi}_{2} \mathrm{Te}_{3}$ unit cell on a silicon substrate . . . 37

$4.1 \quad$ MOKE signal difference as function of pump-probe delay and its FFT amplitude $\ldots \ldots \ldots \ldots \ldots \ldots \ldots \ldots \ldots$

4.2 Kittel and PSSW modes on a continuous 50-nm CoFeB film . . . . 41

4.3 Spatially-resolved magnetization dynamics on the interface between a magnonic crystal and continuous film $\ldots \ldots \ldots$. . . . 44

4.4 Magnetization dynamics on a 5 rows defect line (waveguide) in a magnonic crystal $\ldots \ldots \ldots \ldots \ldots \ldots \ldots$ 
4.5 Demagnetization peak and FFT amplitude of Kittel and PSSW modes on a magnonic waveguide $\ldots \ldots \ldots . \ldots 47$

4.6 The dependence of DE, PSSW and Kittel mode frequencies on film thickness ..................... . . 49

4.7 Magnetization dynamics and Gilbert damping on 82.5-nm CoFeB

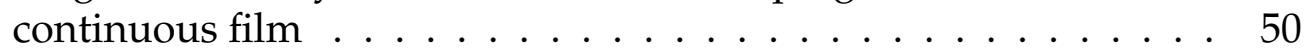

4.8 Magnetization dynamics in a magnonic crystal $(\mathrm{a}=3.5 \mu \mathrm{m}, \mathrm{d}=$ $1 \mu \mathrm{m})$ on 82.5-nm CoFeB film $\ldots \ldots \ldots \ldots$. . . . . . . . 51

4.9 Gilbert damping in a magnonic crystal $(a=3.5 \mu \mathrm{m}, \mathrm{d}=1 \mu \mathrm{m})$ on 82.5-nm CoFeB film . . . . . . . . . . . . . 51

4.10 Reflectivity dynamics on a continuous 50-nm CoFeB film . . . . . 53

4.11 Reflectivity dynamics on a magnonic crystal $(\mathrm{a}=1.75 \mu \mathrm{m}, \mathrm{d}=$ $0.5 \mu \mathrm{m})$ in the $50-\mathrm{nm}$ CoFeB film $\ldots \ldots \ldots \ldots 3$

4.12 Reflectivity dynamics on a magnonic crystal $(\mathrm{a}=700 \mathrm{~nm}, \mathrm{~d}=$ $200 \mathrm{~nm}$ ) in a 50-nm CoFeB film . . . . . . . . . . . 54

4.13 Magnetization dynamics on $(a=1.75 \mu \mathrm{m}, \mathrm{d}=0.5 \mu \mathrm{m})$ and $(a=700 \mathrm{~nm}, \mathrm{~d}=200 \mathrm{~nm})$ magnonic crystals with an external magnetic field applied along the lattice constant $\ldots \ldots$. . . . 56

4.14 Calculated (mumax) effective magnetic field in magnonic crystals 57

4.15 Calculated amplitude of effective field between antidots as function of distance in a unit cell $\ldots \ldots \ldots . \ldots 58$

4.16 Magnetization dynamics in $(a=1.75 \mu \mathrm{m}, \mathrm{d}=0.5 \mu \mathrm{m})$ magnonic crystal, with an external magnetic field applied at $45^{\circ}$ to the lat-

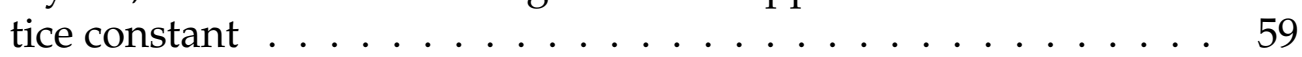

4.17 Magnetization dynamics in $(a=700 \mathrm{~nm}, \mathrm{~d}=200 \mathrm{~nm})$ magnonic crystal, with an external magnetic field applied at $45^{\circ}$ to the lat-

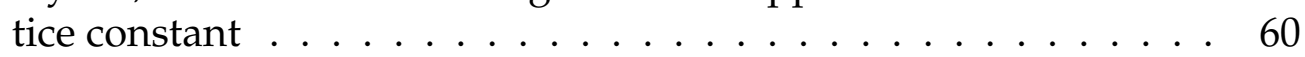

4.18 Simulated temperature distribution on a CoFeB thin film after a laser pump pulse impinges on the surface . . . . . . . . . 62

4.19 Magnetization dynamics on a continuous film across the pump

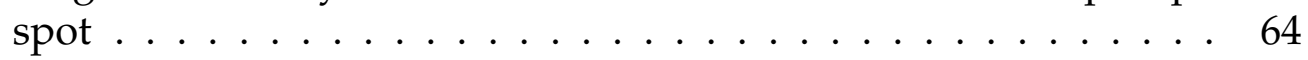

4.20 Magnetization dynamics on a magnonic crystal across the pump

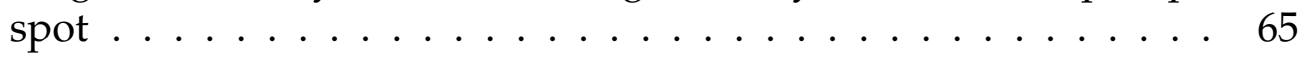

5.1 Reflectivity dynamics on a $31-n m \mathrm{Bi}_{2} \mathrm{Te}_{3}$ film . . . . . . . . . . 68

5.2 Time-resolved reflectivity dynamics on $20 \mathrm{nmCu} / 100 \mathrm{~nm}$ PC bi-

layer and FePtAgCu/NiTa sample . . . . . . . . 70

5.3 Time-resolved reflectivity measurement and simulations on $\mathrm{CoFeB} / \mathrm{MgO}$ and $\mathrm{W} / \mathrm{Si}$ multilayers . . . . . . . . . . . 71

5.4 Reflectivity dynamics on a 200-nm tungsten layer on Si substrate 72

5.5 Time-resolved reflectivity dynamics on 21.6-nm tungsten layer. . . 73 
5.6 Time-resolved reflectivity measurement on $\mathrm{W} / \mathrm{PC}$ double layers on Si substrate . . . . . . . . . . . . . . . . . . 75

$5.7 \quad$ FFT amplitude of correlated reflectivity dynamics on W/PC dou-

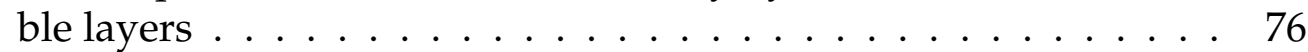

5.8 Simulated elastic strain in W/PC double layers on Si substrate . . 77

5.9 Reflectivity dynamics on $\mathrm{W} / \mathrm{PC}$ multilayer structures $\ldots . . . . .79$

5.10 FFT amplitude of correlated reflectivity signal in W/PC multilayers 80

5.11 FFT amplitude of correlated reflectivity dynamics on $\mathrm{CoFeB} / \mathrm{MgO}$ multilayer with periodic stacking and FFT amplitude of the simulated elastic stress $\ldots \ldots \ldots \ldots$. . . . . . . . . . . 81

5.12 Normalized FFT amplitude of correlated reflectivity dynamics signal on aperiodic $\mathrm{CoFeB} / \mathrm{MgO}$ multilayer and FFT amplitude of the simulated elastic stress as function of depth for front pump

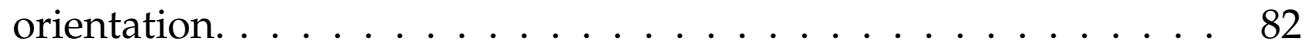

5.13 Normalized FFT amplitude of correlated reflectivity dynamics on a non-commensurate $\mathrm{W} / \mathrm{PC}$ multilayer structure for front and substrate pumping geometries $\ldots \ldots \ldots \ldots$ 


\section{Chapter 1}

\section{Introduction}

Since 1980 many new, magnetism related phenomena have been successfully exploited in modern technology: giant magneto-resistance, magnetic tunnel junctions, spin valves, domain wall motion and many more. Among these, magnonics is an emergent field, that deals with spin-wave quanta or magnons as carriers of information, in analogy to photonics and electronics ${ }^{1-3}$. One of the most promising trends in this field are artificial materials ${ }^{4}$, featuring periodic variations of magnetic properties, i.e magnonic crystals.

Generally, structuring is the most widely used approach for tailoring electromagnetic, phonon and spin-wave properties in solids. Phonons are the particles responsible for heat (together with electrons in metals) and sound transmission, so that understanding and modifying their properties in artificial materials might facilitate hypersound (in GHz range) and heat transfer (in $\mathrm{THz}$ range) control at the nanoscale ${ }^{5}$. Multilayers are periodic structures composed of layers with periodically varying elastic properties, i.e one-dimensional phononic crystals. One of their most interesting properties is low thermal conductivity, arising from suppressed phonon transport due to multiple scatterings at the interfaces. An additional reduction of thermal conductivity may result from using disordered or acoustically mismatched multilayers ${ }^{67}$. In any case, the engineering of thermal properties in nano-structured multilayers requires an understanding of the underlying phonon behavior ${ }^{8}$ as function of the acoustic impedance of the constituting materials. Also, the breaking of multilayer periodicity might influence the elastic wave propagation by suppressing the buildup of constructive interference. In this work we will explore both the acoustic mismatch and aperiodicity strategies in phonon manipulation.

The general trend today in magnonics is to aim at propagating spin-waves with short wavelengths in the range of hundreds of nanometers, due to the ever growing demands in miniaturization. As this process goes on, the interplay between phononic and magnonic degrees of freedom must necessarily be 
taken into account. Moreover, high-frequency surface acoustic waves can be generated by ultrafast laser excitation of nanoscale patterned surfaces ${ }^{9}$. An important example of such a structure is an antidot lattice, which consists of a periodic array of holes in an otherwise continuous ferromagnetic film and that can be regarded both as a phononic ${ }^{10}$ and a magnonic ${ }^{11}$ two-dimensional crystal in $\mathrm{GHz}$ regime.

An alternative approach to lithography patterning in magnonics is using optically reconfigurable magnetic materials 12 , consisting of an optically induced two-dimensional thermal landscape that modifies the saturation magnetization in a periodic manner. Thus, also heating effects must be taken into account when dealing with optically excited magnetization dynamics in magnonic crystals.

In this work, we have exploited many advantages of the all-optical, highintensity experimental setup to investigate both magnetization and elastic dynamics in nano-patterned thin films and multilayers. High-intensity ultrafast lasers offer the possibility of exciting both magnetization and elastic dynamics, by generating a picosecond heat gradient. When the laser fluence is high enough, a heat gradient persisting for up to $1 \mathrm{~ns}$ can be created, which allows to investigate the spin-wave properties in thermal landscapes. Moreover, the excited dynamics can be probed using non-destructive, contactless optical techniques in a time-resolved manner, by making use of the photoelastic and the magneto-optical Kerr effects.

In Chapter 2 we present a brief introduction to the main concepts needed to understand magnetization and elastic dynamics. We start with the description of static magnetization properties in thin, continuous CoFeB films and continue with spin waves in bulk materials and thin ferromagnetic films. As last point, the main properties of two-dimensional magnonic crystals (particularly antidot lattices) are discussed. In the same chapter, elastic wave properties are discussed and the concept of acoustic impedance is presented. As the next level of complexity, we present the reflection coefficient from a slab of soft material embedded between hard material layers, introducing the concept of acoustic mismatch. Then, we calculate the band structure of elastic waves in $\mathrm{CoFeB} / \mathrm{MgO}$ and $\mathrm{W} / \mathrm{PC}$ multilayers, and discuss the bulk and surface mode properties. As next point, the details of the thermal generation and the propagation characteristics of strain pulses in opaque materials are addressed. The chapter closes with a discussion of thermal excitation of magnetization dynamics in CoFeB thin films.

The experimental setup is discussed using Jones matrix formalism in Chapter 3. The magneto-optical Kerr effect is approached in a phenomenological model, allowing to determine the relation between the measured optical signal 
and the magnetization dynamics. The chapter closes with a brief description of the multilayer structures and magnonic crystal samples, including patterning techniques.

In Chapter 4, the main results of magnetization dynamics on sub-micron antidot lattices are discussed. As a first point, we address spatially-resolved measurements of magnetization dynamics on different topologies, such as the interface between a magnonic crystal and continuous film, and on magnonic waveguides. Next, we focus on the Gilbert damping in a magnonic crystal where two different spin-wave modes at the same frequency. The chapter closes with a discussion of elastic and magnetization dynamics on antidot lattices with a high aspect ratio between its thickness and inter-antidot distance.

In Chapter 5, a discussion of the main results on elastic dynamics in multilayers with high (W/PC) and low $(\mathrm{CoFeB} / \mathrm{MgO})$ acoustic mismatch is provided. The effects of breaking the multilayer periodicity is also addressed. Moreover, two different excitation geometries are compared: transmission and reflection.

Finally, in Chapter 6 the most relevant findings are summarized and an outlook to further experiments is presented. 


\section{Chapter 2}

\section{Theoretical considerations}

In this chapter, a brief overview of the main concepts in magnetization and elastic dynamics is provided. In both cases, we discuss spin and elastic waves as elementary magnetic and mechanic excitations respectively. Later on, we discuss how these dynamics properties can be manipulated by periodically structuring the underlying material, i.e producing phononic and magnonic crystals. As the last point, ultrafast optical excitation of elastic and spin waves is discussed.

\subsection{Ferromagnetic ordering}

In magnetic materials, the magnetic ordering occurs due to the interaction of neighboring spins or, in general, magnetic moments. In the particular case of ferromagnets, their spins tend to align parallel to each other when the temperature is low enough, and consequently these materials exhibit a net magnetization. This long-range magnetic ordering decreases with increasing temperature, reaching a paramagnetic state at a critical (Curie) temperature.

The interaction that leads to magnetic ordering in a material was first formulated by Heisenberg; here we follow the discussion as provided in Refs. 13-16. Magnetic order arises from the antisymmetry condition for the total wave function of Fermi particles. When two electrons are sufficiently close, the overlapping of their individual wavefunctions leads to an energy difference between $S=0$ and $S=1$ states ( $S$-total spin). This energy difference for a whole system of electrons can be written as the exchange (Heisenberg) Hamiltonian 17/18:

$$
\mathcal{H}_{e x}=-\sum_{i, j} J_{i j}\left(S_{i} \cdot S_{j}\right)
$$

where $S_{i}$ and $S_{j}$ are the spin-operators of electrons at sites $i$ and $j$ respectively, and $J_{i j}$ is the exchange constant that depends on the overlapping of electron 
wavefunctions.

Electrons can also be thought of as magnetic dipoles, carrying a momentum $g \mu_{\mathrm{B}} S_{i}$, interacting with each other:

$$
\mathcal{H}_{\text {dip }}=\left(g \mu_{B}\right)^{2} \sum_{i, j}\left(\frac{S_{i} \cdot S_{j}}{r_{i j}^{3}}-3 \frac{\left(S_{i} \cdot r_{i j}\right)\left(S_{j} \cdot r_{i j}\right)}{r_{i j}^{5}}\right)
$$

where $r_{i j}$ is the magnitude of the vector connecting sites $i$ and $j, \mu_{B}$ is the Bohr magneton and $\mathrm{g}$ - Lande factor.

The exchange interaction is short-range and larger (in magnitude) than the dipolar interaction, so that it typically determines the magnetic ordering and the static magnetization properties of a material. On the other hand, dipolar interaction becomes important when dealing with spin waves or magnetic excitations at long wavelengths (compared to the atomic lattice).

Further energy contributions may arise from a particular geometrical arrangement of the spins and their interaction with the lattice atoms through spinorbit coupling, giving rise to the so-called magneto-crystalline anisotropy. Due to the complexity of this problem, it is often treated phenomenologically $\frac{13}{119}$ by representing the corresponding Hamiltonian as series expansion in powers of components of the spin-operator and retaining only the terms required by the lattice symmetry. The simplest case is the uniaxial anisotropy:

$$
\mathcal{H}_{\mathrm{uni}}=\sum_{\mathrm{i}} \mathrm{K}_{\mathrm{u}}\left(\mathrm{S}_{\mathrm{i}, z}\right)^{2}
$$

where $K_{u}$ is the uniaxial anisotropy constant and $S_{i, z}$ is the $z$-component of the spin operator at site $i$.

Moreover, also applied stress can change the energy landscape through a coupling to magnetic properties of the material.

Although the microscopic description is often helpful for the basic understanding of magnetic phenomena, throughout this work, the macroscopic approach is more convenient due to the fact that we will typically deal with length scales much larger than the atomic lattice of the material. In this approach, not individual magnetic moments are considered but a sum over many sites, defining a local magnetization:

$$
M(r)=g \mu_{B} \sum_{i} S_{i} \delta\left(r-r_{i}\right)
$$

Thus, in the rest of this work, the microscopical treatment of spin wave properties is abandoned and all relevant phenomena are described in a macroscopic approach. 


\subsubsection{Static magnetization properties}

In this section the static magnetization of a saturated, ferromagnetic thin film is discussed, taking into account the uniaxial anisotropy properties of $\mathrm{CoFeB}$ thin films.

Macroscopically, the static magnetization configuration of a ferromagnetic system is determined by the total free energy $F_{t o t}$, to which contribute macroscopic equivalents of Hamiltonians discussed in the previous section. The effective magnetic field in the sample is defined by $\overrightarrow{\mathrm{H}}_{\text {eff }}=-\frac{\partial \mathrm{F}_{\text {tot }}}{\partial \overrightarrow{\mathrm{M}}}$. In equilibrium and saturation, the magnetization vector $\vec{M}$ and $\vec{H}_{\text {eff }}$ are collinear. The resulting direction of $\overrightarrow{\mathrm{H}}_{\text {eff }}$ can be determined as the orientation where the minimum of free energy occurs.

In a thin CoFeB film, the effective field has the following contributions:

$$
\overrightarrow{\mathrm{H}}_{\text {eff }}=\overrightarrow{\mathrm{H}}_{\text {ext }}+\overrightarrow{\mathrm{H}}_{\mathrm{dem}}+\overrightarrow{\mathrm{H}}_{\mathrm{uni}}
$$

where $\overrightarrow{\mathrm{H}}_{\text {ext }}$ is the external applied magnetic field, $\overrightarrow{\mathrm{H}}_{\mathrm{dem}}$ - the demagnetizing field, and $\overrightarrow{\mathrm{H}}_{\mathrm{uni}}$ - uniaxial anisotropy field.

Since $\mathrm{CoFeB}$ is amorphous, the uniaxial anisotropy cannot arise as a result of a magneto-crystalline anisotropy. Instead, it can result from strain or spinorbit interactions at the surface of the thin film, or volume contributions whose origins are still debated 2021 . However, its functional form is identical to that of a demagnetizing field and it has an important contribution to the total energy. As will be shown later in section 2.3.2, it also plays an important role when exciting magnetization dynamics using ultrafast optical pulses.

In Fig. 2.1 the free energies corresponding to each effective field term are plotted, as function of the angle between the sample plane normal and the magnetization vector. The free energy corresponding to demagnetization and uniaxial anisotropy have a minimum in the sample plane. The Zeeman energy (due to external magnetic field) has a minimum when the external field direction and magnetization vector are collinear. Thus, when an applied magnetic field is canted with respect to the sample plane, two competing terms arise: the uniaxial and demagnetizing fields, that tend to pull the effective field in-plane, and the external field, that pulls the effective field out of the sample plane. The result is a minimum of total free energy that is slightly $\left(\sim 2^{\circ}\right)$ out of plane.

It is also interesting to note that when the external field is applied strictly in-plane, only the Zeeman energy term remains with a minimum in the sample plane. 


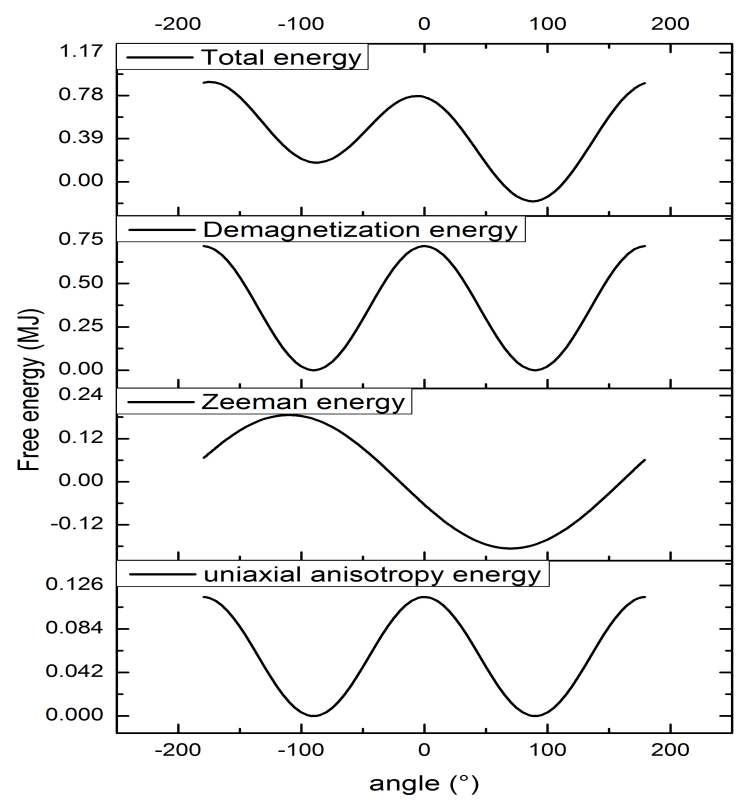

Figure 2.1: Calculated free energy of a $\mathrm{CoFeB}$ thin film with external magnetic field applied at $20^{\circ}$ to the sample plane, $M_{s}=1.8 \mathrm{~T}$ and $K_{u}=-2.2 \times 10^{4} \frac{\mathrm{J}}{\mathrm{m}^{3}}$. The $x$-axis is the angle between the sample plane normal and the magnetization vector.

\subsubsection{Spin waves in bulk materials}

The idea of a spin wave was first established by Bloch, as a slight deviation from a (static) equilibrium position of interacting magnetic moments traveling through the lattice. This deviation could be caused by an initial perturbation to the orientation of a magnetic moment. Thus, the concept is analogous to atomic oscillations around the equilibrium positions in a solid. When the quantum nature of these magnetization oscillations is accounted for, the spin waves are referred to as magnons, in analogy to phonons as quantized lattice vibrations.

Spin waves in bulk (unbound) materials have been thoroughly investigated both experimentally and theoretically 22 . The semi-classical approach is based upon the idea that magnetic moments are precessing vectors in an effective magnetic field $\overrightarrow{\mathrm{H}}_{\text {eff }}$ satisfying the Landau -Lifshitz torque equation ${ }^{23}$.

$$
\frac{\partial}{\partial t} \vec{M}=-\gamma \vec{M} \times \overrightarrow{H_{e f f}}
$$

with $\gamma$ - gyromagnetic ratio. It must be noted that this equation neglects damping effects that can be taken into account by adding, on the right hand side, the term $\frac{\alpha}{M} \frac{\partial}{\partial t} \vec{M} \times \vec{M}$, where $\alpha$ is a phenomenological (Gilbert) damping parameter 
and determines the energy dissipation per precession period, when the interaction between spin-wave modes is not taken into account ${ }^{24}$.

By using the explicit form of $\vec{H}_{\text {eff }}$ in the linearized Eq. 2.6, and taking into account the exchange field contribution, an expression for the spin wave precession frequency can be found ${ }^{25}$ :

$$
\omega^{2}=\left(\omega_{H}+\omega_{A}\right)\left(\omega_{H}+\omega_{A}+\omega_{M} \sin ^{2} \theta_{k}\right)
$$

where $\omega_{H}=\gamma \mu_{0} H_{e f f}, \omega_{M}=\gamma \mu_{0} M, \omega_{A}=\gamma \frac{2 A}{M} k^{2}$ with $A$ - macroscopic exchange constant, and $\theta_{k}$ - the angle between $\vec{M}$ and the k-vector, that characterizes the anisotropic propagation of spin waves.

A particular case, $\theta_{k}=0$ and $k=0$, that is a uniform magnetization precession is often referred to as Kittel mode ${ }^{\sqrt{26}}$ described by the equation:

$$
\omega_{\text {Kittel }}^{2}=\omega_{H}\left(\omega_{H}+\omega_{M}\right)
$$

It must be noted that, in general, the frequencies of bulk spin waves are in the range from $w=w_{H}\left(\theta_{k}=0\right)$ to the Kittel mode frequency, that sets the upper limit.

\subsubsection{Spin waves in thin films}

Spin wave properties in a magnetic thin film are determined by the boundary conditions imposed by the finite size of the film in one of its dimensions. As will be seen in this section, new modes localized near the surface, as well as bulk modes that satisfy the surface boundary conditions are found.

Two remarkable features of the spin wave spectrum must be mentioned. Firstly, the frequency of surface spin waves depends not only on the orientation of its wave vector, but also on the magnitude. This will have important consequences when working with magnonic crystals, as it will allow us to identify the wave vector magnitude (and thus relate it to the periodicity of the magnonic crystal) by measuring the spin-wave frequency only. The second feature is a quantization of bulk modes due to the finite thickness of the film.

There are many geometries for spin wave propagation in thin films, determined by the relative orientation of the film plane and its magnetization vector. However, due to anisotropy constrains in our magnetic samples, that force the magnetization to orient practically in-plane, only this geometry will be discussed in what follows. This way we can define the wave vector of the propagating spin wave as $k=\left(k_{\|}, k_{\perp}, k_{z}\right)$ where $k_{\|}\left(k_{\perp}\right)$ is the wave vector component parallel (perpendicular) to $\vec{M}$ and $k_{z}$ is the component normal to the sample plane. 
The case of static magnetization lying in the plane of film surface has been solved for wave vectors whose in-plane magnitude $\mathrm{K}=\left|\mathrm{k}_{\|}, \mathrm{k}_{\perp}\right|$ satisfies $\mathrm{K} \gg$ $\omega / c$, where $c$ is the speed of light and $\omega$ is the spin-wave frequency, i.e the magnetostatic regime discussed by Damon and Eshbach 27/28. This yields an infinite series of real solutions for $k_{z}$ that correspond to bulk spin-wave modes and an imaginary solution, that corresponds to a surface spin-wave mode.

When neglecting the exchange interaction in a thin film, the frequency of the surface spin-wave mode, that propagates perpendicularly to the magnetization direction reads 1627 :

$$
\omega_{\mathrm{DE}}^{2}=\omega_{\mathrm{H}}\left(\omega_{\mathrm{H}}+\omega_{M}\right)+\frac{\omega_{M}^{2}}{4}\left(1-e^{-2 k_{\perp} \mathrm{d}}\right)
$$

where $d$ is the film thickness. It must be noted that the frequency of this mode lies in the interval $\omega_{\text {Kittel }} \leqslant \omega_{D E} \leqslant \sqrt{\omega_{\text {Kittel }}^{2}+\frac{\omega_{M}^{2}}{4}}$.

Moreover, for the in-plane wave vector $k$ at $45^{\circ}$ to $\vec{M}$, the dispersion relation reads:

$$
\omega_{45}^{2}=\omega_{H}^{2}+\frac{\omega_{H} \omega_{M}}{2}\left(1+\frac{1}{k d}\left(1-e^{-k d}\right)\right)+\frac{\omega_{M}^{2}}{2 k d}\left(1-e^{-k d}\right)\left(1-\frac{1}{k d}\left(1-e^{-k d}\right)\right)
$$

Here again we can note the anisotropic character of spin-wave propagation also for surface modes. Both of these surface modes have an exponentially decaying amplitude profile into the sample thickness.

On the other hand, the frequency of the bulk mode with no in-plane wavevector components or perpendicular standing spin wave mode (PSSW) reads:

$$
\omega_{\mathrm{PSSW}}^{2}=\left(\omega_{\mathrm{H}}+\omega_{\mathrm{A}}\right)\left(\omega_{\mathrm{H}}+\omega_{\mathrm{A}}+\omega_{\mathrm{M}}\right)
$$

where $\omega_{A}=\gamma \frac{2 A}{M} k_{z}^{2}, k_{z}=n\left(\frac{\pi}{d}\right)$ with $n=0,1,2 \ldots \infty$. This mode is identical with the bulk modes in an infinite sample (Eq. 2.7) with a quantized $k_{z}$.

It is interesting at this point to address the Gilbert damping parameter as presented in the Eq. 2.6. Although the dispersion characteristics of spin waves have been calculated neglecting $\alpha$, it can be introduced through an absorptionlike coefficient in the resonance frequency:

$$
\alpha=\frac{1}{\tau \omega \frac{\partial \omega}{\partial H}}
$$

where $\tau$ - the spin wave decay time and $\omega$ - the resonance frequency of the particular spin-wave mode. 


\subsubsection{Magnonic crystals}

We have seen in previous sections that magnetization dynamics in a thin film follows a rich, anisotropic spectrum. In addition to these characteristics, further enhancement and control over the propagation of spin waves can be achieved by introducing a periodic modulation of internal magnetic field properties in the film plane. Such micro- or nanostructured materials are called magnonic crystals. In this section we focus on 2D magnonic crystals, consisting of a periodic array of holes (antidot lattice) on CoFeB thin films, and their properties. An extensive review on magnonic crystals can be found in Ref. 29
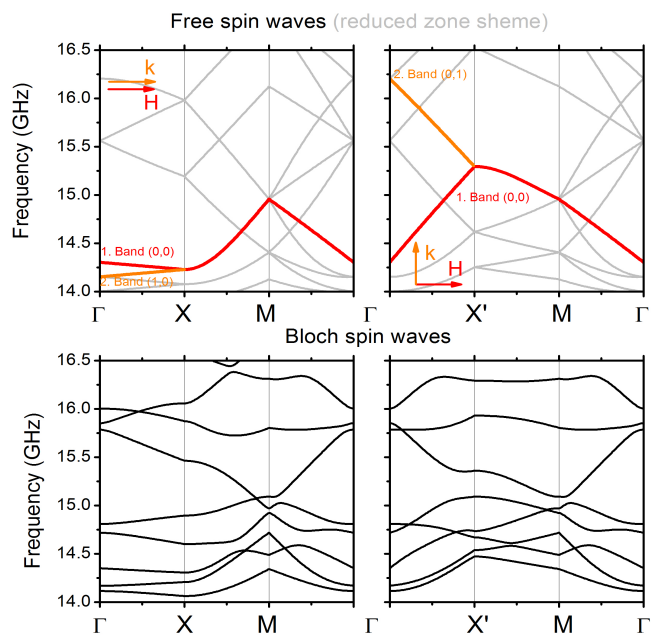

Figure 2.2: Calculated band structure of spin waves in an antidot lattice on CoFeB thin film ${ }^{30}$. Image provided by Henning Ulrichs.

Due to the complexity of calculating the spin-wave dispersion in a magnonic crystal, it is usually approached by numerical methods, namely the plane wave approximation or micromagnetic simulations. The Landau-Lifshitz equation (Eq. 2.6) is solved taking into account a periodical modulation of magnetization and under adequate boundary conditions ${ }^{30}$. In analogy to free electrons in a periodic potential and phonons in multilayer systems, this modulation of magnetic properties induces a band structure with full band gaps where spin waves are not allowed to propagate ${ }^{1}$. As shown in Fig. 2.2, in the free spinwave model, where the radius of the antidot $R \rightarrow 0$, the folding of continuous film spin-wave bands in Damon-Eshbach $\left(k \perp H_{e f f}\right)$ geometry and for $k \| H_{e f f}$ is observed. When antidots with finite radius are considered, a band gap opens at the Brillouin zone boundary.

In general, many parameters determine the resulting spin-wave band struc- 
ture, such as the periodicity of the antidot lattice (lattice constant) ${ }^{11}$, the orientation of external magnetic field with respect to magnonic crystal high-symmetry directions $\frac{\sqrt{30}}{}$, the shape and size of the antidots $\sqrt{31}$, underlying material properties, etc.

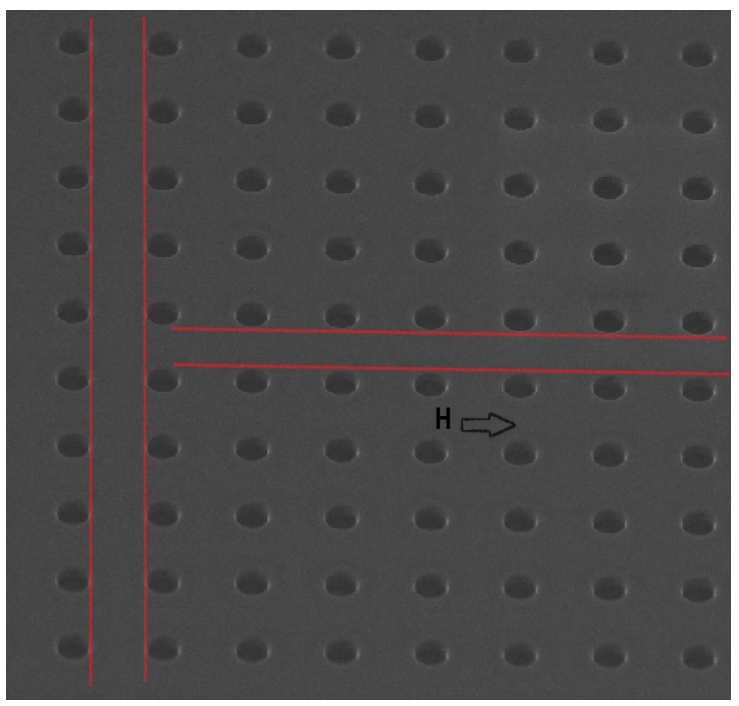

Figure 2.3: A scanning electron microscopy image of an antidot lattice in CoFeB matrix. In red, two main channels for spin-wave propagation are marked. The in-plane direction of the applied magnetic field $\mathrm{H}$ is marked with an arrow.

The antidot size and shape modify the internal field in the thin film, due to the demagnetizing fields near the antidot edges. This large variation of internal field can be viewed as a potential well that confines the low-frequency modes, known as edge modes, with typical frequencies in the order of few gigahertz ${ }^{11}$.

A useful way of thinking about a magnonic crystal is as a collection of ferromagnetic nanowires contained between antidot rows, so that the nanowire width is defined by the distance between the antidots. In correspondence to the symmetry directions of a square lattice, these nanowires can be magnetized perpendicularly or along their long axis, as shown in Fig. 2.3. In a square antidot lattice generally two types of spin-wave modes in the nanowire channels are distinguished $32+-34$.

- Extended modes, in the nanowires oriented perpendicularly to the applied magnetic field (in Damon-Eshbach geometry). This mode extends through the lattice. The internal field and magnetization are non-uniform across the nanowire, so that two main modes are possible: i) a DamonEshbach-like mode, with $\mathrm{k} \perp \mathrm{H}$, propagating in the middle of the nanowire where the internal field is largely uniform and, ii) a mode propagating 
along the edges of the nanowire where large variations of the internal field exist, if the antidots are sufficiently close, so that the dipolar fields of nearest-neighbors antidots connect with each other ${ }^{35}$.

- Localized modes, between the neighboring antidots in the nanowire oriented with its long axis parallel to the external magnetic field. In this geometry, the magnetic field is oriented along the easy axis of the nanowire, so that the internal field is largely uniform. This presents a possibility of standing spin-wave quantized modes in the smallest cross-sectional direction. Usually, in thin film samples, this direction is across the sample thickness, so that quantized bulk modes (Eq. 2.11) of an unpatterned thin film are found 1130 .

For the spin-wave modes quantized in the transversal (to the nanowire long axis) direction, the effective width of the nanowire, due to modified pinning conditions on the nanowire edges, must be taken into account ${ }^{36}$. The pinning parameter $d(p)$ is then defined as:

$$
\begin{aligned}
d(p) & =\frac{2 \pi}{p[1+2 \ln (1 / p)]^{\prime}} \\
p & =t / w
\end{aligned}
$$

where $w$ is the width of the nanowire i.e., the lattice parameter, $t$ is the film thickness, and $p$ - the aspect ratio. The role of the pinning parameter is to minimize the dipolar interaction of the induced surface charges on the lateral edges of the nanowire. Thus, the effective width of the nanowire becomes:

$$
w_{\text {eff }}=w \frac{d}{d-2}
$$

It must be noted that, for small aspect ratios $p \rightarrow 0$, the effective width approaches the real width of the nanowire.

In $\mathrm{CoFeB}$ antidot lattices it was demonstrated that the patterning induces a Damon-Eshbach-like mode with a wave-vector magnitude $|\vec{k}|=\frac{\pi}{a}$ where $a-$ lattice period. Reducing the lattice period to $1.5 \mu \mathrm{m}$ produces localized modes that dominate the spin-wave spectrum ${ }^{11}$. In general, the aspect ratio of the nanowires has a large influence on the frequencies of both propagating and localized modes, as has been recently shown ${ }^{37}$. For small aspect ratios $p<<1$, both localized and propagating modes approach the Kittel frequency of nonpatterned films, since the demagnetizing fields become negligible in both orientations of the nanowire. On the other hand, for large values of $p$, the demagnetizing fields become more pronounced so that the spin-wave frequencies in the 
perpendicularly magnetized nanowire and edge modes should decrease, while the frequency of localized modes in the longitudinally magnetized nanowires increases.

This way, spin-wave modes can localize in the nonuniform regions of internal magnetic field ${ }^{38-40}$, imposed by the particular antidot configuration geometry and the direction of the external magnetic field, since the field nonuniformity is the greatest along its direction.

Another feature of magnonic crystals is the possibility of introducing lattice defects i.e., by skipping few rows of antidots or changing some other lattice parameters such as the antidot size. This could further enhance spin wave propagation and dispersion characteristics, in analogy to a photonic crystal ${ }^{1}$. One of the most interesting aspects is spin-wave localization along a defect line ${ }^{41}$ or symmetry breaking for the spin-wave propagation ${ }^{42}$. Moreover, the localization of spin waves can result not only from the non-uniformity of the internal magnetic field, but also as a direct consequence of the magnonic spin-wave band structure ${ }^{43}$, for example on a defect line in a magnonic crystal (waveguide) or at the edge (interface) of a finite magnonic crystal.

\subsection{Elastic dynamics in multilayers}

In a continuous medium, in the long-wavelength approximation, phonons can be treated as elastic waves. A plane acoustic wave in $1 \mathrm{D}$, propagating in $z$ direction in an infinite medium, is described by solving the simple wave equation 4445 :

$$
\begin{array}{r}
\frac{\partial \sigma_{z z}}{\partial z}=\rho \frac{\partial^{2} u_{z}}{\partial t^{2}} \\
\mathfrak{u}_{z}(z, t)=\int_{+\infty}^{z} \eta_{z z}\left(z^{\prime}, t\right) d z^{\prime}
\end{array}
$$

where $\sigma_{z z}$ and $\eta_{z z}$ are the longitudinal stress and strain in $z$-direction, $u_{z}$ is the displacement in $z$-direction, $\rho$ is the material density. The exact solution is determined by the boundary and initial conditions of the problem. However, a general solution in an isotropic material can be written as:

$$
\mathfrak{u}_{z}(z, \mathfrak{t})=a e^{i \omega t}\left(e^{i k z}+e^{-i k z}\right)
$$

where $a$ is the displacement amplitude, $k$ - wave vector, and $\omega$ - wave frequency.

Upon reflection on an interface, an elastic wave is transmitted and reflected as any other classical wave. The boundary conditions generally require the 
continuity of displacement $u_{z}$ (or displacement velocity) and stress at the interface. Imposing these conditions on the incident, reflected and transmitted waves with corresponding amplitudes $a_{i}, a_{r}$ and $a_{t}$, yields the reflection and transmission coefficients at the interface between two materials $\mathrm{A}$ and $\mathrm{B}$, independent of $\omega$ and $k$ :

$$
\begin{aligned}
& r_{A, B}=\frac{a_{i}}{a_{r}}=\frac{Z_{A}-Z_{B}}{Z_{A}+Z_{B}}, \\
& t_{A, B}=\frac{a_{i}}{a_{t}}=\frac{Z_{A}}{Z_{A}+Z_{B}} .
\end{aligned}
$$

The indexes $\mathrm{A}$ and $\mathrm{B}$ indicate the corresponding material, while the acoustic impedance $Z$ is a material property that depends on the mass density and the longitudinal, sound velocity $\nu_{l}$ as:

$$
\mathrm{Z}=\rho v_{l}
$$

The next level of complication is reached when considering the reflection and transmission not from an interface, but a slab of material (characterized by $\rho, v_{l}$ ) with a thickness $d$ embedded between semi-infinite media $A\left(\rho_{A}, v_{A}\right)$ and $B$ $\left(\rho_{B}, v_{B}\right)$. In this case, the reflection and transmission coefficients are not constant as in the simplest case, but depend on the frequency of incident wave, because some frequencies will induce resonances in the slab.

A simple approach to solving this problem is applying the impedance translation theorem ${ }^{46}$ to the layer. For an elastic wave traveling in the media A towards the slab, the effective impedance of the slab and the media behind it, $Z_{\text {input }}$, can be calculated as:

$$
Z_{\text {input }}=Z_{0} \frac{Z_{B}-i Z_{0} \tan \left(k_{0} d\right)}{Z_{0}-i Z_{B} \tan \left(k_{0} d\right)}
$$

Then, the reflection coefficient of stress wave amplitude on the slab is calculated as:

$$
R=\frac{Z_{\text {input }}-Z_{A}}{Z_{A}+Z_{\text {input }}}
$$

Applying Green's theorem formalism to an infinite, periodic multilayer of slabs $A$ and $B$ characterized by the respective acoustic impedances $Z_{A}$ and $Z_{B}$, thicknesses $d_{A}$ and $d_{B}$ and longitudinal wave velocities $v_{A}$ and $v_{B}$, the dispersion relation can be written as $47+49$ :

$$
\cos (k D)=C_{A} C_{B}-0.5 S_{A} S_{B}\left(\frac{Z_{A}}{Z_{B}}+\frac{Z_{B}}{Z_{A}}\right)
$$

where $C_{A, B}=\cos \left(\omega \frac{d_{A, B}}{v_{A, B}}\right), S_{A, B}=\sin \left(\omega \frac{d_{A, B}}{v_{A, B}}\right)$ and $D=d_{A}+d_{B}$. 


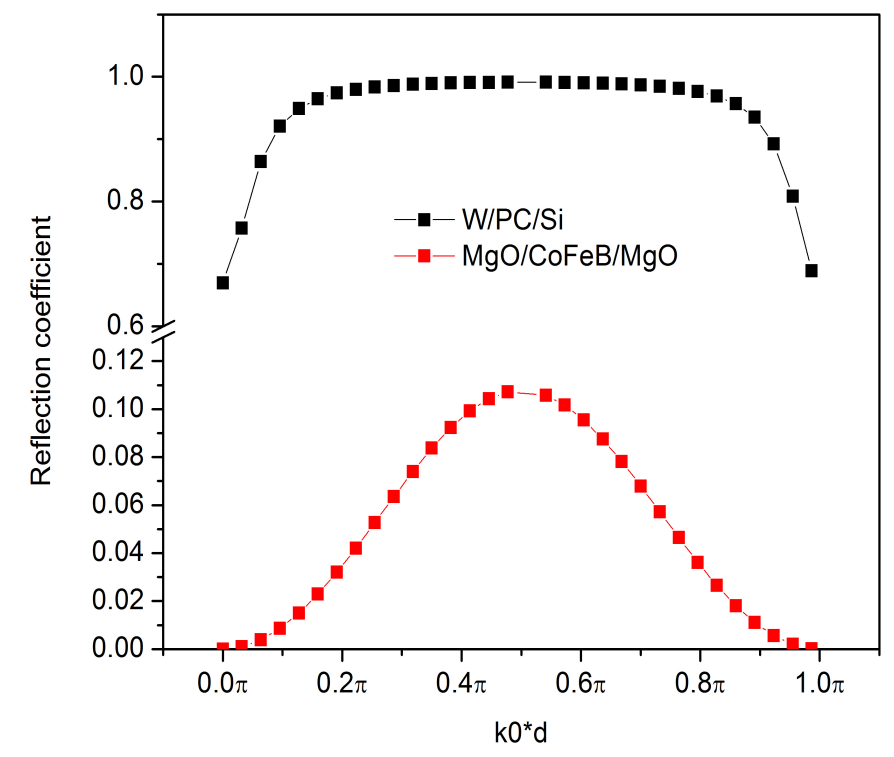

Figure 2.4: Absolute value of the reflection coefficient of a slab of thickness $d$ embedded between two media, as indicated in the legend. $k_{0}$ is the wave vector of the incident wave.

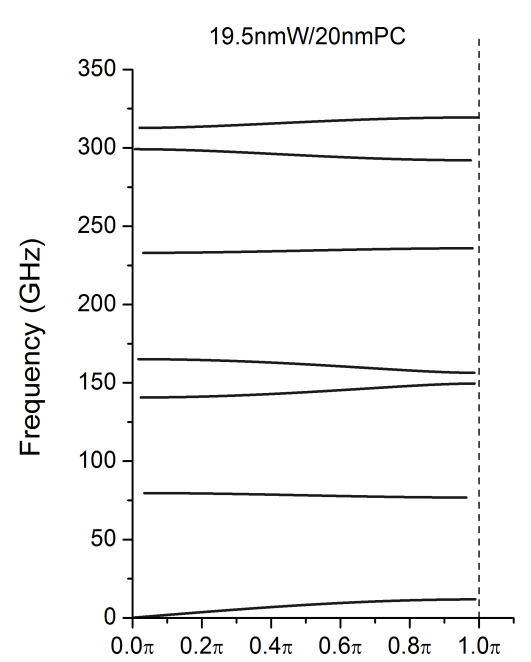

kD

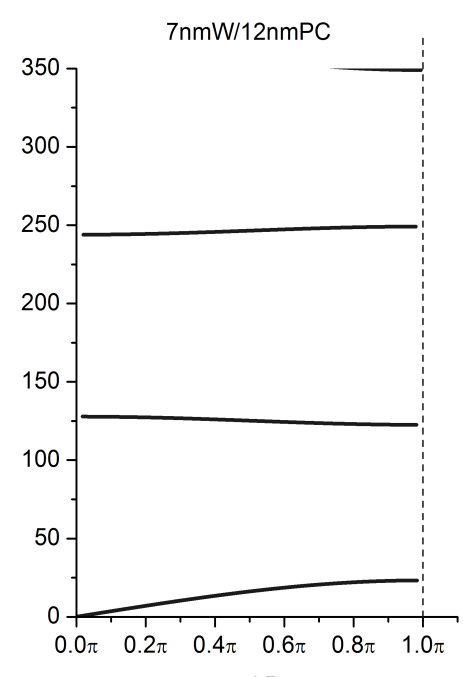

$\mathrm{kD}$

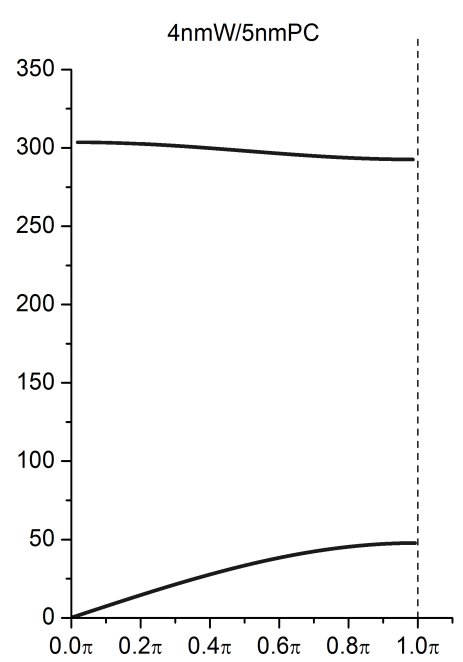

kD

Figure 2.5: Calculated dispersion relation of an elastic wave in an infinite W/PC multilayer with different layer thicknesses. The legend above each panel indicates the layer thickness of the corresponding material. Dotted line at $k \mathrm{D}=\pi$ indicates the edge of the first Brillouin zone, where D - total thickness of a unit cell. 


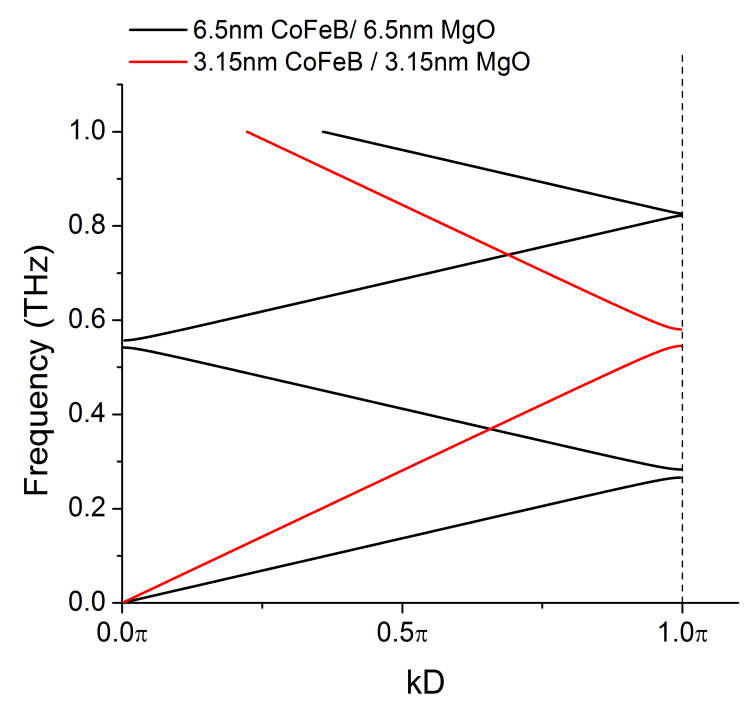

Figure 2.6: Calculated dispersion relation of an elastic wave in an infinite 6.5 $\mathrm{nm} \mathrm{CoFeB/} 6.5 \mathrm{~nm} \mathrm{MgO}$ multilayer (black line) and $3.15 \mathrm{~nm} \mathrm{CoFeB/3.15} \mathrm{nm}$ $\mathrm{MgO}$ multilayer (red line). Dotted line at $\mathrm{kD}=\pi$ indicates the first Brillouin zone edge, where $\mathrm{D}$ - total thickness of a unit cell.

This equation has been evaluated for multilayers of soft/hard materials (see Fig. 2.5) and, for multilayers of materials with similar acoustic impedances (Fig. 2.6) and with different periodicities. As expected, both cases present a linear dispersion relation when $\mathrm{kD}<<\pi$, and a band gap opening near the Brillouin zone edge. However, in materials with similar acoustic impedances, the band gap is smaller than in hard/soft multilayers. This can be explained by the fact that, as we have previously seen, soft layers acoustically isolate the hard layers, so that a wider band-gap is expected. As the thickness of the layers decreases, the band-gap width is enhanced and moreover, higher frequencies in the first branch are reached.

For finite lattices with $\mathrm{N}$ cells, the dispersion relation is not continuous and only $\mathrm{N}-1$ bulk modes are available, that satisfy the condition ${ }^{47}$.

$$
\begin{array}{r}
\sin (N k D)=0 \\
k D=\frac{m \pi}{N}, m=1,2,3 \ldots N-1
\end{array}
$$




\subsection{Excitation mechanism}

The excitation of both coherent magnetization oscillations and elastic dynamics, in this work, are initiated by a transient electron heating. When the highfluence pump beam impinges on the surface of a metal, the energy is first absorbed by the charge carriers. This creates a high-energy electron population (with $\approx 1.5 \mathrm{eV}$ excess energy), that reaches a quasi-thermal equilibrium distribution after a characteristic time of tens of femtoseconds. In addition, in metals the electron bands that might be optically coupled are often spin-degenerate, meaning that no net spin-dependent excitation takes place. The carrier density is not expected to change because the orbital momentum is practically negligible in this system. This way, the main effect of the pump is the heating of the carrier population, which leads to a heat transfer to the lattice system on a timescale of a few picoseconds, as proposed by Beaurepaire in the threetemperature model ${ }^{50}$.

In the next sections the excitation mechanism that triggers coherent elastic and magnetization oscillation, and its most important features are discussed.

\subsubsection{Elastic dynamics}

In this section, the picosecond generation, propagation and detection characteristics of strain pulses in opaque materials are discussed, as developed by Thomsen et al in Ref. 51. The simplest model for the conversion of laser-deposited energy into heat assumes that this process is instantaneous and the energy deposition follows the optical penetration profile into the sample i.e., the heat diffusion is neglected. In most cases, this is a valid approximation, since the pump energy is absorbed very rapidly, as discussed above, and the heat diffusion process usually takes $\sim 100 \mathrm{~ns}-10 \mu \mathrm{s}$. As mentioned in section 3.1, the experimental measurement windows spans $1 \mathrm{~ns}$ after the excitation, so that in general, heat diffusion must be addressed in a different experimental approach, appropriate for longer timescales, such as time-domain thermo-reflectance (TDTR) ${ }^{52}$.

The optical pump spot size is much larger $(\approx 30 \mu \mathrm{m})$ than the optical absorption depth in opaque films $(\approx 10-50 \mathrm{~nm})$, so that only the acoustic propagation normal to the surface of the film is considered, reducing the problem to one dimension (z). A notable exception are the in-plane patterned thin films where also the surface acoustic waves can be excited. However, in this section we only consider multilayer systems that can reflect the strain pulse back to the surface, where it is detected as a change in reflected intensity by the second (probe) pulse.

The thermal expansion tensor of a solid $\alpha_{i j}$ is defined by $\eta_{i j}=\alpha_{i j} \Delta T$, where $\eta_{i j}$ is the strain tensor and $\Delta T$ is the z-dependent change in temperature, com- 
pared to before the arrival of the pump pulse. For small displacements along z-direction $\mathfrak{u}_{z}$, the strain z-component is given by: $\eta_{z z}=\frac{\partial u_{z}}{\partial z}$. In turn, the relation between stress and strain, in presence of a thermal strain, is given by ${ }^{45}$.

$$
\sigma_{z z}=\rho v_{l}^{2} \eta_{z z}-3 \mathrm{~B} \alpha \Delta \mathrm{T}(z)
$$

where $B$ is the bulk modulus.

To solve the elastic wave equation for zero body-forces (Eq. 2.16) the explicit form of the temperature profile must be stated. In an homogeneous, isotropic solid the profile of energy deposited by an ultrashort optical pulse on the surface is:

$$
W(z)=(1-R) \frac{Q}{A \zeta} e^{(-z / \zeta),}
$$

where $\mathrm{R}$ is the reflectivity coefficient $(\mathrm{R} \approx 0.5$ for tungsten at $800 \mathrm{~nm}), \mathrm{Q}$ - energy per pulse $(\mathrm{Q} \approx 280 \mathrm{~nJ}$ for the amplified laser pulse), $\zeta$ - optical penetration depth ( $\zeta \approx 23 \mathrm{~nm}$ for tungsten), and $A$ - excitation area defined by the Gaussian pump spot. The temperature profile can be calculated taking into account the material specific heat capacity $\mathrm{C}: \Delta \mathrm{T}(z)=\mathrm{W}(z) / \mathrm{C}$.

Substituting the explicit stress expression (Eq. 2.25) in Eq. 2.16 yields a simple wave equation for $\mathfrak{u}_{z}$ with a source term:

$$
\frac{\partial^{2} u_{z}}{\partial t^{2}}=v_{l}^{2} \frac{\partial^{2} u_{z}}{\partial z^{2}}+\frac{v_{l}^{2} \eta_{0}}{\zeta} e^{-\frac{z}{\zeta}}
$$

The solution to this problem is determined by the initial and boundary conditions. First, the boundary condition is given by the absence of any restoring force on the surface, so that there is no stretching perpendicular to the surface at the surface itself: $\sigma_{z z}=0, z=0$. Substituting this condition into Eq. 2.25, it's found that, for every $t>0$, the strain at the surface is constant:

$$
\eta_{0}=\frac{3 \mathrm{~B} \alpha(1-\mathrm{R}) \mathrm{Q}}{\mathrm{AC \zeta \rho v_{l } ^ { 2 }} .}
$$

The initial conditions are the absence of strain and changes of strain in the film before the arrival of the pump pulse: $\eta_{z z}=0, \frac{\partial \eta_{z z}}{\partial t}=0$ and $u_{z}=0$.

Thus a solution can be found for $z>053$ :

$$
\eta_{z z}=\eta_{0} e^{-\frac{z}{\zeta}}-\frac{\eta_{0}}{2}\left[e^{-\frac{z+v_{l} t}{\zeta}}+e^{-\frac{\left|z-v_{l} t\right|}{\zeta}} \operatorname{sgn}\left(z-v_{l} t\right)\right] .
$$

This strain profile contains a time-independent term, that follows an exponential with a decay length $\zeta$ - the characteristic optical penetration depth. The time-dependent term (in square brackets) is a strain pulse propagating at the 
speed of sound in the material, that consist of two exponentials with opposite signs i.e., compressive and tensile deformations. Since the time independent strain produced by the thermal expansion does not leave the surface of the material, it will not produce a time-dependent change in the optical constants i.e., it will not be detected in the reflectivity dynamics experiment. The additional propagating strain pulse will periodically change the optical constants in the volume of the material, if reflected back from a surface.

The spectral content of the propagating elastic strain pulse is calculated by applying the Fourier transform to the time-dependent component of Eq. 2.29. taking the center of the pulse as the origin of coordinates ${ }^{53}$.

$$
\eta(\omega)=\int_{-\infty}^{\infty} \eta_{z z}(z, t) e^{i \omega t} d t=\eta_{0} \frac{i \omega}{\omega^{2}+\left(v_{l} / \zeta\right)^{2}} .
$$

Thus, the spectral bandwidth of excited strain pulse is defined by the sound velocity and the optical penetration depth.

In transparent materials, strain cannot be induced by thermal expansion. However, both unipolar and bipolar strain profiles can be induced by an opaque transducer layer $\frac{5455}{}$, and the reflected strain-wave amplitude depends on the relative acoustic impedances of the transducer and the sample.

In more complicated structures, such as multilayers, multiple reflections from different interfaces and a modulated excitation profile (due to different absorption coefficients in each layer) must be considered if the optical penetration depth is larger than the total multilayer thickness. In our experiments, multilayers are composed of both opaque and transparent materials. The thickness of some layers is as small as $\sim 5 \mathrm{~nm}$, so that the whole multilayer stack is within the optical penetration depth of pump and probe beams. In this case, the thermal strain profile is almost constant across an individual layer. This makes necessary the application of numerical simulation techniques to calculate the thermal strain profile, although these often rely on the assumption that the optical and material properties of very thin layers are similar to the bulk values ${ }^{56}$.

Some additional strain contributions may arise from carrier diffusion in semiconductor films, such as Si substrate ${ }^{51}$. Additionally, when the in-plane symmetry of the sample is broken, the excitation of both longitudinal and normal stress waves must be addressed ${ }^{57}$.

The optical detection scheme relies on the strain-dependent changes in the optical constants of the material, that result in a change of the optical reflection coefficient. Due to the imposed boundary condition of zero-stress at the free surface, and as can be seen in Eq 2.29, there is no time-dependent variation of strain at the free surface, even when the strain pulse echoes back and forth in the sample. However, due to a finite, non-zero optical penetration depth, a volume 
of the sample is probed, so that in general $[58$.

$$
\Delta R(t) \propto \int f(z) \eta_{z z}(z, t) d z
$$

where $f(z)$ is a sensitivity function, that defines how the refractive index depends on strain. In general, the optical reflectivity is defined by the Fresnel relations through the dielectric constant $\varepsilon$, that depends on the complex refractive index, with $n$ - refraction and $k$ - absorption coefficient ${ }^{59}$. If the strain induced changes $\Delta \varepsilon$ are small:

$$
\begin{aligned}
\varepsilon & =(n+i k)^{2} \\
\Delta \varepsilon(z, t) & =2(n+i k)\left(\frac{\partial n}{\partial \eta}+i \frac{\partial k}{\partial \eta}\right) \eta(z, t) .
\end{aligned}
$$

This way, $f(z)$ must be a function of the photoelastic constants $\frac{\partial n}{\partial \eta}$ and $\frac{\partial k}{\partial \eta}$, that are material- and wavelength-dependent. Additionally, $f(z)$ will depend on probe polarization, angle of incidence and its optical frequency bandwidth.

In transparent materials, no absorption takes place, so that the reflectivity changes arise from the interference of the probe light with the light reflected form the propagating elastic pulse ${ }^{5460}$. Furthermore, in multilayer systems interface motion may also lead to probe interference effects ${ }^{53}$, still yielding strainproportional changes in the refractive index.

In view of this, we can conclude that the picosecond laser ultrasonics is an adequate technique for the study of multilayer structures of opaque and transparent materials, that provides a large excitation bandwidth (Eq. 2.30) and, coupled to a signal-averaging experimental techniques, is a sensitive detection of elastic dynamics.

\subsubsection{Magnetization oscillations}

After the initial laser-induced ultrafast demagnetization, lattice heating can trigger magnetization oscillations in a ferromagnet ${ }^{6162}$ on a timescale of several nanoseconds. This process can be understood without taking into account the details of ultrafast demagnetization phenomenon, if the demagnetization process is assumed due to spin-flip events 63 . Thus, the laser induces a random reorientation of local spins within the laser penetration depth and the demagnetization profile within the sample follows the optical penetration profile. The local spins are assumed to interact with each other via the exchange interaction only. As shown by simple micromagnetic simulations ${ }^{63}$, shortly after the optical excitation, spin waves with large wave vector are excited $\left(\mathrm{k} \approx 0.1 \mathrm{~nm}^{-1}\right)$ 
and gradually decay into spin waves with shorter wave vectors i.e., spin-wave modes with a lower energy.

Thus, this spin-wave excitation technique is very broadband, although it does not feature wave vector selectivity. Rather, the spin-wave band structure itself selects which modes are excited, by populating them according to their density of states. This is specially interesting in the context of a magnonic crystal, which allows to tailor the spin-wave band structure by the tuning of lattice parameters and the direction of the applied magnetic field. However, even in continuous films, a rich spin-wave spectrum can be excited, featuring both surface and bulk spin-wave modes due to the asymmetry of optical excitation normal to the film plane 64 .

In a macroscopic approach, the excitation of magnetization oscillations can be thought of as stemming from a magnetic field pulse generated by a very fast $(\approx 20 \mathrm{ps})$ temperature change in the ferromagnetic sample ${ }^{61}$. In terms of Eq. 2.5, some contributions to the effective magnetic field, namely the uniaxial anisotropy field, are temperature dependent. This way, the deposited laser energy is transferred to the lattice and raises its temperature, so that the value of the uniaxial anisotropy constant $K_{\mathfrak{u}}(T)$ changes, thus tipping the magnetization out of its initial equilibrium position. In order to achieve this, it is necessary that the magnetization and effective magnetic field are slightly canted out-of-plane.

The rapid change in the temperature-dependent anisotropy field (i.e., the so-called anisotropy field pulse) changes the direction of the effective internal magnetic field for a very short time (less than 20 ps). Although initially the magnetization is aligned with the effective field, now they become non-collinear. This creates a torque acting on the magnetization vector, that launches a coherent magnetization precession. After this, the heat is transferred to the lattice, so that the anisotropy field and consequently, the effective internal magnetic field return to their initial equilibrium direction. When the laser power is sufficiently high, the ultrashort pulse also creates a local temperature gradient in the sample. This way, the laser can be used not only to excite and probe magnetization dynamics, but also to create a spin-wave trap 65 . 


\section{Chapter 3}

\section{Methods}

This chapter focuses on the description of the experimental setup used for timeresolved measurements of magnetization dynamics and coherent elastic dynamics. Although these phenomena involve different physical processes, they can be addressed in the same experimental setup with only slight modifications. This is due to the versatility of magneto-optical setup, that can provide additional information about the time-dependent reflectivity changes in the sample, simultaneously to the magnetization dynamics. Firstly, we focus on understanding the experimental implementation of ultrafast magneto-optical setup using Jones matrix formalism, as well as the signal processing. Later on, we discuss the relation of measured optical signal (Kerr angle) to the magnetization dynamics. Along the same line, the description of the experimental setup for reflectivity dynamics measurement and the interpretation of the resulting optical signal, is provided.

The chapter concludes with a detailed description of sample preparation and structuring. In particular, the influence of ultrafast laser heating on $\mathrm{CoFeB}$ magnetic properties and antidot milling by focused ion beam are addressed. Furthermore, the suitability of multilayer structures for experimental studies is shown through the analysis of important parameters such as the interface roughness, layer quality and thickness.

\subsection{Experimental implementation}

An essential piece of the experimental setup is the $80 \mathrm{MHz}$ oscillator laser (Coherent, MirA 9000), providing ultrashort laser pulses (central wavelength $\lambda_{c}=$ $780-820 \mathrm{~nm}$, corresponding to $1.55 \mathrm{eV}$ photon energy, and pulse duration of $40 \mathrm{fs}$ ) that guarantee a high temporal resolution. A further pulse energy amplification by means of a regenerative amplifier (Coherent, RegA 9040) with $250 \mathrm{kHz}$ 
repetition rate, is essential to excite the desired physical processes i.e., magnetization or elastic dynamics. The laser beam of short, amplified pulses is divided into a high-intensity pump beam ( $~ 95 \%$ of total intensity), that excites the dynamical processes, and a low-intensity beam $(\sim 5 \%)$ used to probe the dynamics in the sample. So, a contactless, all-optical excitation and detection scheme is achieved, where spatial resolution is limited only by the optical diffraction limit. Moreover, pump and probe beams can be displaced with respect to each other, simply by tilting an end mirror with a piezocontroller. This is particularly useful for magnonics experiments, where a spatial profile of precession frequency or amplitude often provides additional information on spin-wave properties. The pump pulse can be delayed with respect to the probe pulse by a up to $1 \mathrm{~ns}$ delay stage (Physik Instrumente) with a $5 \mathrm{fs}$ time resolution. In combination with short laser pulses, this provides a time-resolved insight on short and long timescale dynamics.

The pump pulse is used to initiate the dynamics. In experiments on magnetic films, it starts a magnetization precession by thermally inducing a reorientation of the magnetic easy axis and an additional strain pulse, thus exciting both coherent and incoherent phonons. In order to distinguish the initial state (no pump) and the corresponding excited state, in all experiments the pump beam is modulated at $800 \mathrm{~Hz}$ by a mechanical chopper; this way, no absolute values of corresponding physical quantities (i.e magnetization or reflectivity), but only their changes are detected. The detection of magnetization state also requires a modulation of the probe beam polarization at $50 \mathrm{kHz}$ by a photoelastic modulator (PEM-90, Hinds) as explained further in this chapter.

As shown in Fig. 3.1a, initially the probe beam is polarized in the optical plane (Edmond Optics, Glan-Thompson polarizer) and converted to circularly polarized light by a quarterwave retarder plate. For magnetization dynamics measurements, the polarization of incident light is then periodically modulated by a PEM. The polarization plane of probe beam reflected by the sample is rotated proportionally to its magnetization: this is the so-called magneto-optical Kerr effect. Finally, polarization changes are converted into a change of beam intensity using a second (analyzer) polarizer, which axis is set perpendicular to the optical plane. The change of beam intensity is detected by a slow diode as a voltage and filtered at PEM frequency by the lock-in amplifier (Stanford Research Systems, SR 830 DSP), providing information about the net state of magnetization in the sample. The signal is then passed on to a second lock-in amplifier and filtered at chopper frequency, in order to measure the signal difference between the pump-on and pump-off states. This double modulation technique (at PEM and chopper frequencies) ensures low signal-to-noise ratio and high sensitivity of the measured signal, reducing the noise that originates 
from polarization fluctuations caused by changes in Fresnel reflectivity coefficients. However, it does not reduce mechanical noise (for example, due to delay stage vibrations). Therefore, the performance of this technique strongly depends on the stability of phase and amplitude modulation by the PEM.

The procedure described above is done for every delay stage position, allowing a time-resolved measurement of the excited dynamics in the sample.

On the other hand, excited phonons in the sample directly produce changes in the intensity of reflected light, due to a varying refraction index as result of heat-induced stress. This way, in order to detect coherent phonons, no polarization modulation is necessary as can be seen in Fig. 3.1b.

In the next section, a quantitative description of the experimental setup using Jones calculus formalism is provided.

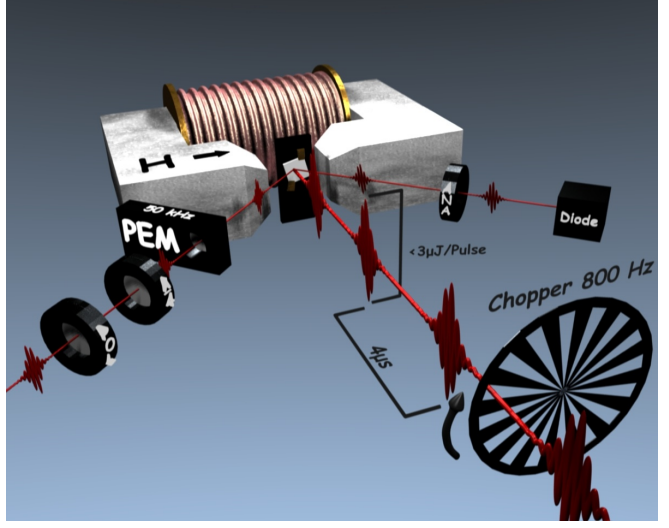

(a)

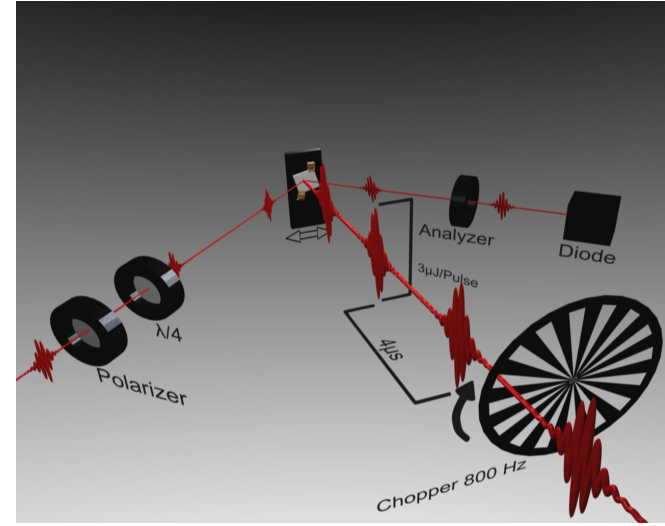

(b)

Figure 3.1: Experimental setup for the detection of (a) magnetization oscillations and (b) reflectivity dynamics. Images provided by Jakob Walowski.

\subsection{Jones calculus formalism}

In this section, we provide an quantitative analysis of the differential signal obtained by the experimental assembly, shown in Figs. 3.1b and 3.1a, using Jones matrix formalism 66 . Every optical element in the setup can be described by a $2 \times 2$ Jones matrix. Depolarization, absorption and reflection on optical elements are neglected and a perpendicular incidence is assumed. The amplitudes of electric field parallel $\left(E_{p}^{\prime}\right)$ and perpendicular to the plane of incidence $\left(E_{s}^{\prime}\right)$ after passing through the optical element are given by $\left(\begin{array}{l}E_{p}^{\prime} \\ E_{s}^{\prime}\end{array}\right)=M\left(\begin{array}{l}E_{p} \\ E_{s}\end{array}\right)$ 
where $E_{p}$ and $E_{s}$ are incident electric field amplitudes and $M$ is the Jones matrix of an optical element.

In general, the Jones matrix for a linear polarizer with the transmission axis oriented at an angle $\alpha$ to the plane of incidence is:

$$
\left(\begin{array}{cc}
\cos ^{2} \alpha & \sin \alpha \cos \alpha \\
\sin \alpha \cos \alpha & \sin ^{2} \alpha
\end{array}\right)
$$

so that a polarizer oriented in $\hat{\mathrm{p}}$-plane is described by: $\mathrm{P}=\left(\begin{array}{ll}1 & 0 \\ 0 & 0\end{array}\right)$, and the analyzer in ŝ-plane: $A=\left(\begin{array}{ll}0 & 0 \\ 0 & 1\end{array}\right)$. by:

On the other hand, the Jones matrix for a quarter-wave retarder $\mathrm{Q}$ is given

$$
Q=\frac{1}{\sqrt{2}}\left(\begin{array}{cc}
1+i \cos (2 \theta) & i \sin (2 \theta) \\
i \sin (2 \theta) & 1-i \cos (2 \theta)
\end{array}\right),
$$

where $\theta$ is the angle of fast axis with respect to the $\hat{p}$-plane.

The main function of the PEM is to modulate the relative phase velocity of $\hat{s}$ and $\hat{p}$-polarized light at a frequency $\omega_{M} / 2 \pi=50 \mathrm{kHz}$. The associated Jones matrix is: $O=\left(\begin{array}{cc}e^{i \frac{\phi}{2}} & 0 \\ 0 & e^{-i \frac{\phi}{2}}\end{array}\right)$, where the retardation $\phi=\phi_{0} \sin \left(\omega_{M} t\right)$ is timedependent, with $\phi_{0}$-retardation amplitude. An analytic expression of this term requires the following expansion of $\cos (\phi(t))$ and $\sin (\phi(t))$ in Fourier series:

$$
\begin{array}{r}
\cos (\phi)=\mathrm{J}_{0}\left(\phi_{0}\right)+2 \mathrm{~J}_{2}\left(\phi_{0}\right) \cos \left(2 \omega_{M} \mathrm{t}\right)+\ldots+\text { higher order terms } \\
\sin (\phi)=2 \mathrm{~J}_{1} \sin \left(\omega_{M} \mathrm{t}\right)+\ldots+\text { higher order terms }
\end{array}
$$

where $J_{k}\left(\phi_{0}\right)$ is k-order Bessel function. Since we are using the lock-in technique, only the first and second harmonics of $\omega_{M}$ are of interest.

The magneto-optical Fresnel reflection matrix for an arbitrary sample is: $R=\left(\begin{array}{cc}\tilde{r}_{p p} & \tilde{r}_{p s} \\ \tilde{r}_{s p} & \tilde{r}_{s s}\end{array}\right)$, where $r_{i j}$ is the ratio of the incident j-polarized and the reflected i-polarized electric field. In particular, $\tilde{r}_{p p}=r_{p p} e^{i \delta_{p p}}$ and $\tilde{r}_{s s}=r_{s s} e^{i \delta_{s s}}$ are the complex Fresnel coefficients describing optical reflectivity and the offdiagonal elements $\tilde{r}_{p s}=-\tilde{r}_{s p}=r_{p s} e^{i \delta_{p s}}$ account for magneto-optical Kerr effect or optical birefringence. In this notation, $r_{i j}$ is the magnitude and $\delta_{i j}$ is the phase of the complex number $\tilde{r}_{i j}$.

Finally, the intensity on the diode photodetector is given by $\mathrm{I}=\mathrm{E}^{\prime 2}$. 


\subsubsection{Reflectivity dynamics detection setup}

We begin by quantitatively describing the detection setup for reflectivity dynamics, where no modulation of the probe beam is required and the Jones matrix description is greatly simplified. Hence, the PEM is omitted and the total transmission through the optical elements is given by:

$$
\begin{aligned}
\left(\begin{array}{c}
E_{p}^{\prime} \\
E_{s}^{\prime}
\end{array}\right)=A \cdot R \cdot Q \cdot P & \left(\begin{array}{c}
E_{p} \\
E_{s}
\end{array}\right) \\
& =\frac{1}{\sqrt{2}}\left(\begin{array}{cc}
0 & 0 \\
\tilde{r}_{s p}+i\left(\tilde{r}_{s p} \cos (2 \theta)+\tilde{r}_{s s} \sin (2 \theta)\right. & 0
\end{array}\right)\left(\begin{array}{c}
E_{p} \\
E_{s}
\end{array}\right) .
\end{aligned}
$$

Assuming that the materials we use are not birefringent (i.e., $\tilde{\mathrm{r}}_{\mathrm{sp}} \approx 0$ ), the final intensity on the diode, as function of Fresnel coefficients, is calculated as:

$$
\mathrm{I}_{\text {refl }}=\frac{1}{2} \mathrm{E}_{\mathrm{p}}^{2} \mathrm{r}_{\mathrm{ss}}^{2} \sin ^{2}(2 \theta)=\mathrm{I}_{0} \mathrm{r}_{\mathrm{ss}}^{2} \sin ^{2}(2 \theta) .
$$

Finally, setting $\theta=45^{\circ}$ (i.e the probe beam incident on the sample is circularly polarized) we obtain the maximum intensity on the diode, directly proportional to $r_{s s}$. The reflectivity $r_{p p}$ can be measured in an analogous way, by setting the analyzer transmission axis along the $\hat{p}$-axis. However, these measurements generally have a worse signal-to-noise ratio, because the pump beam is also $\hat{p}$ polarized, so that some spurious pump light is always present in this signal. Moreover, the magnitude of reflectivity $r_{s s}$ is always greater than $r_{p p}$ in any material, therefore we have chosen $r_{s s}$ for our measurements.

\subsubsection{Magneto-optical Kerr effect detection setup}

In this section we provide a brief introduction to the magneto-optical Kerr effect ${ }^{67}$ (MOKE) and a phenomenological analysis of our setup for measuring the Kerr rotation and ellipticity within Jones matrix formalism. Here we focus on the modulation technique used for MOKE measurements, where a modulation of probe beam by a photoelastic modulator is necessary.

\section{MOKE phenomenological description}

The magneto-optical Kerr effect is a very useful probing tool for magnetization properties of magnetic materials, especially thin films, due to its large surface sensitivity. It manifests itself as a change of polarization plane or intensity of incident light after the reflection on the surface of a magnetized material, proportionally to the magnetization of the sample. This way, linearly polarized 
light experiences a rotation of polarization plane (Kerr rotation $\theta_{k}$ ) and a phase difference between the electrical field $\hat{s}$ and $\hat{p}$-components (Kerr ellipticity $\epsilon_{k}$ ). Generally, this defines a complex Kerr angle:

$$
\tilde{\Theta}_{k}=\theta_{k}+i \epsilon_{k} .
$$

Several types of MOKE are distinguished, depending on the relative orientation of the magnetization and the plane of incidence:

- Polar MOKE - Magnetization in the plane of incidence and perpendicular to the sample plane.

- Longitudinal MOKE - Magnetization in the sample plane and in the plane of incidence.

- Transverse MOKE - Magnetization in sample plane and perpendicular to the plane of incidence.

as shown in Fig 3.2

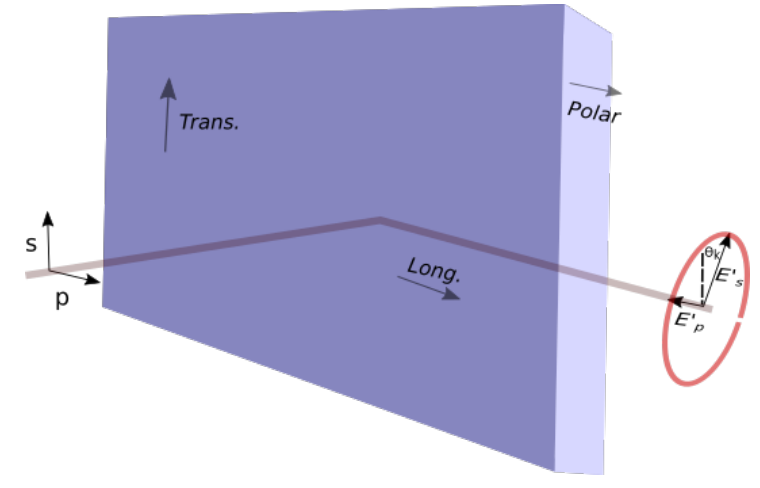

Figure 3.2: Definition of the coordinate system and a schematics of polar, longitudinal and transverse MOKE.

It must be noted that it is possible to define MOKE as purely polar, longitudinal, or transversal, only when the magnetization is static. Since we are interested in magnetization dynamics, during which magnetization precesses or changes its magnitude, we will necessarily detect a time-dependent combination of these three effects.

In accordance to the Maxwell equations, the propagation of electromagnetic waves through a medium, in absence of external charge current, is given by:

$$
\nabla \times \nabla \times \vec{E}=-\frac{1}{c^{2}} \frac{\partial^{2} \vec{D}}{\partial t^{2}},
$$


where the electrical displacement vector $\vec{D}$, the electric field $\vec{E}$ and polarization $\vec{P}$ are related by the constitutive relation: $\vec{D}=\vec{E}+4 \pi \vec{P}$. In the frequency domain, considering a local response to monochromatic excitation, the polarization is related to the electric field by a susceptibility tensor: $\vec{P}(\omega)=\chi(\omega) \vec{E}(\omega)$. Hence,

$$
\vec{D}(\omega)=\varepsilon(\omega) \vec{E}, \quad \varepsilon(\omega)=1+4 \pi \chi(\omega),
$$

so that the second-rank polar tensor $\varepsilon$ can be understood as the dielectric constant that fully describes the linear optical response of the medium. In general, the elements of this tensor are complex numbers: $\varepsilon_{i j}=\varepsilon_{i j}^{\prime}+i \varepsilon_{i j}^{\prime \prime}$.

Moreover, the interaction of electromagnetic field with an isotropic magnetized sample is represented by the local dielectric tensor 6869 :

$$
\varepsilon=\varepsilon_{x x}\left(\begin{array}{ccc}
1 & -i Q m_{z} & i Q m_{y} \\
i Q m_{z} & 1 & -i Q m_{x} \\
-i Q m_{y} & i Q m_{x} & 1
\end{array}\right)
$$

where $\varepsilon_{x x}=\varepsilon_{z z}$ is assumed for simplicity, $\mathrm{Q}=\mathfrak{i} \frac{\varepsilon_{x y}}{\varepsilon_{x x}}$ is the complex magnetooptical constant, and $m_{x}, m_{y}$ and $m_{z}$ are the direction cosines of magnetization vector $\vec{M}_{s}$ of the sample.

In must be noted that the diagonal elements transform symmetrically upon time reversal (i.e., when magnetization changes to the opposite direction). This is the normal linear optical behavior of any (non-magnetized) material, that defines the usual complex refractive index. However, the off-diagonal terms, proportional to the magnetization, break the time-reversal symmetry and transform anti-symmetrically upon magnetization reversal. This accounts for the material magneto-optical properties, such as the Kerr effect. The off-diagonal terms imply different microscopic response of the magnetized medium to the propagation of right and left circularly polarized light i.e., the dielectric constant of the material (and consequently the complex refractive index) depends on the polarization of incident light. This difference in refractive index leads to different phase velocities and absorption coefficients of the normal modes, and consequently, to a polarization rotation and ellipticity upon reflection from a magnetized sample surface ${ }^{70}$.

Since the perpendicular component of magnetization $m_{x}$ yields no contribution within the first order of $Q$, the magneto-optical response of an arbitrarily magnetized sample comes mainly from parallel and perpendicular (to the plane of incidence) magnetization components. This way, the Kerr angle of an optically thick medium, in the general case of arbitrary magnetization orientation 
and oblique incidence, can be written as follows ${ }^{71}$ :

$$
\begin{aligned}
\phi_{k}^{p} & =\frac{\tilde{r_{p s}}}{r_{\tilde{p} p}^{\tilde{p}}}=\frac{\cos \left(\theta_{0}\right)\left(m_{z}+m_{y} \tan \left(\theta_{1}\right)\right)}{\left.\cos \left(\theta_{0}+\theta_{1}\right)\right)} \cdot \frac{i n_{0} n_{1} Q}{\left(n_{1}^{2}-n_{0}^{2}\right)^{\prime}} \\
\phi_{k}^{s} & =\frac{\tilde{r_{p}}}{\tilde{r_{s s}}}=\frac{\cos \left(\theta_{0}\right)\left(m_{z}-m_{y} \tan \left(\theta_{1}\right)\right)}{\left.\cos \left(\theta_{0}-\theta_{1}\right)\right)} \cdot \frac{i n_{0} n_{1} Q}{\left(n_{1}^{2}-n_{0}^{2}\right)^{\prime}},
\end{aligned}
$$

where $\theta_{0}, \theta_{1}, n_{0}, n_{1}$ are the angles of incidence and refraction and the refractive indexes of non-magnetic and magnetic media, respectively.

The refractive angle $\theta_{1}$ can be determined using Snell's law, provided that the complex refractive indexes of both media are known. In particular, for $40 \mathrm{~nm} \mathrm{CoFeB}$ film on thermally oxidized $\mathrm{SiO}_{2}$ we find ${ }^{72}: \mathrm{n}_{1}=3.5+i 4.2$ at $800 \mathrm{~nm}$ and $\mathrm{n}_{0}=1$ in the air. In our setup the angle of incidence of probe beam $\theta=35^{\circ}$, so that using Snell's law $\theta_{1} \approx 13^{\circ}$. This means that the longitudinal MOKE contribution (proportional to $m_{y}$ ) to the overall Kerr rotation is reduced, compared to the polar MOKE, by a factor of $\tan \left(\theta_{1}\right) \approx 0.23$ (see Eq. 3.10). This makes the polar Kerr effect the dominant contribution.

This way, the Kerr angle is expressed as a simple function of optical parameters of the media and magnetization and a second factor, namely the polar Kerr effect for normal incidence, also linearly proportional to sample magnetization. Thus, by measuring either Kerr rotation or ellipticity, magnetization changes in the sample can be monitored. The experimental determination of Kerr rotation and ellipticity, which is not a trivial task, is treated in the next section.

The magnetooptical constant $\mathrm{Q}$ has not been experimentally determined for $\mathrm{CoFeB}$ to the best of my knowledge, since it implies a careful measurement of the dielectric tensor elements that depend strongly on exact material parameters and stoichiometry. This prevents us from estimating the absolute value of Kerr angle, and only the changes in it (i.e., changes in magnetization) are discussed. Typically, Kerr rotation and ellipticity do not exceed one degree, because the ratio of off-diagonal to diagonal elements is of the order of 0.01 . This makes the development of a reliable low-noise experimental technique for the measurement of Kerr angle very important, which is the main topic of the next section.

\section{Experimental setup}

The MOKE experimental setup includes the modulation of polarization by a PEM. Using Jones matrix formalism, the transmission through optical elements of the setup is given by the equation:

$$
\left(\begin{array}{l}
E_{p}^{\prime} \\
E_{s}^{\prime}
\end{array}\right)=A \cdot R \cdot O \cdot Q \cdot P\left(\begin{array}{l}
E_{p} \\
E_{s}
\end{array}\right) .
$$


The evaluation of Eq. 3.12 is straightforward for a quarter-wave plate orientation $\theta=45^{\circ}$ and yields:

$$
E^{\prime 2}=\frac{E_{p}^{2}}{2}\left(r_{s p}^{2}+r_{s s}^{2}-2 r_{s s} r_{s p}\left(\sin \left(\delta_{s s}-\delta_{s p}\right) \cos (\phi)-\cos \left(\delta_{s s}-\delta_{s p}\right) \sin (\phi)\right)\right) .
$$

Using Eq 3.4 we obtain:

$$
\begin{array}{r}
\mathrm{I}_{0}=r_{s p}^{2}+r_{s s}^{2}-2 r_{s s} r_{s p} J_{0}\left(\phi_{0}\right) \sin \left(\delta_{s s}-\delta_{s p}\right), \\
I_{\omega}=-2 r_{s s} r_{s p} \cos \left(\delta_{s s}-\delta_{s p}\right) J_{1}\left(\phi_{0}\right), \\
I_{2 \omega}=-4 r_{s s} r_{s p} \sin \left(\delta_{s s}-\delta_{s p}\right) J_{2}\left(\phi_{0}\right) .
\end{array}
$$

Kerr rotation $\theta_{\mathrm{K}}$ and ellipticity $\epsilon_{\mathrm{K}}$ are defined as real and imaginary parts of Eq.3.10, namely:

$$
\begin{aligned}
\theta_{s} & =\frac{r_{s p}}{r_{s s}} \cos \left(\delta_{s s}-\delta_{s p}\right), \\
\theta_{p} & =\frac{r_{p s}}{r_{p p}} \cos \left(\delta_{p p}-\delta_{s p}\right),
\end{aligned}
$$

and

$$
\begin{array}{r}
\tan \left(\epsilon_{s}\right)=-\frac{r_{s p}}{r_{s s}} \sin \left(\delta_{s s}-\delta_{s p}\right), \\
\tan \left(\epsilon_{p}\right)=\frac{r_{p s}}{r_{p p}} \sin \left(\delta_{p p}-\delta_{s p}\right) .
\end{array}
$$

so that substituting back into Eq. 3.14 we obtain:

$$
\begin{array}{r}
I_{\omega}=-2 r_{s s}^{2} J_{1}\left(\phi_{0}\right) \theta_{s} \\
I_{2 \omega}=-4 r_{s s} r_{p p} J_{2}\left(\phi_{0}\right) \tan \left(\epsilon_{s}\right)
\end{array}
$$

Hence, the lock-in signals at $\omega_{M}$ and $2 \omega_{M}$ yield directly the Kerr rotation and ellipticity up to a proportionality factor. Moreover, the PEM is operated at a retardation amplitude $\phi_{0}=108^{\circ}$, when measuring rotation, and $\phi_{0}=178^{\circ}$, when measuring ellipticity, that maximize the first and second order Bessel functions respectively and thereby the signal on the diode.

In general, we can write the time-dependent Kerr angle as:

$$
\phi(t)=N(t)+\sum_{i=x, y, z} F_{i}(t) M_{i}(t),
$$


where $N(t)$ is a non-magnetic contribution (symmetric upon reversal of magnetization) that could arise from non-linear optical effects such as, for example, pump-induced optical birefringence and in general, any magnetizationindependent contribution to the off-diagonal terms of the dielectric tensor. In order to eliminate this background in the transient measured signal, we typically consider signal difference for the data analysis:

$$
\Delta \phi(t)=\phi(M(t))-\phi(-M(t)),
$$

where the opposite signs of $M$ are obtained as a result of reversing the external magnetic field. On the other hand, $F(t)$ as seen in the Eq. 3.10 can be thought of as an effective Fresnel coefficient. If the optical constants of the material that determine the value of $F(t)$ (i.e the refractive index) are time-independent even at high pump fluence, we can regard the effective Fresnel coefficient as constant. This way, we can ensure that the optical signal measurements reveal the true magnetization dynamics in the material.

Measuring both real and imaginary parts of the Kerr angle (rotation and ellipticity), rather than $r_{s p}$ (the magnitude of Kerr angle), is particularly important in order to ensure that ultrafast magneto-optical measurements reveal the true magnetization dynamics in the sample. For example, by comparing the Kerr rotation and ellipticity in thin $\mathrm{Ni}$ films ${ }^{73}$, it was demonstrated that non-magnetic contributions dominate the magneto-optical response in the first $500 \mathrm{fs}$ after pump excitation, thus no information about the fast magnetization dynamics could be obtained. However, since our experiments are concerned with long timescale magnetization dynamics (up to 1ns after excitation), long after the optically excited electrons reach the thermodynamic equilibrium distribution, we typically measure only Kerr rotation.

\subsection{Samples: description and characterization}

The following section provides a detailed description of investigated samples (namely magnonic crystals and multilayer structures) and their physical properties, necessary to understand some of the obtained results. Most of the samples investigated in this work are the outcome of collaborations with other groups. W/Si and W/PC multilayer samples were prepared by Florian Döring (Institut für Materialphysik, Universität Göttingen), FePtAgCu/NiTa multilayers were produced by S. Pisana and T. Santos (HGST, USA). Bismuth telluride sample was prepared and characterized in Prof. Gregor Mussler working group (Forschungszentrum Jülich).

$\mathrm{CoFeB} / \mathrm{MgO}$ multilayers and $\mathrm{CoFeB}$ thin films were prepared in our group. Finally, $\mathrm{CoFeB}$ thin films were characterized on Vibrating Sample Magnetome- 
ter (VSM) with assistance from Soham Manni (Uni Göttingen) and magnonic crystal structures were produced using Focused Ion Beam (FIB) by myself.

\subsubsection{Magnonic crystals on thin CoFeB film}

$\mathrm{CoFeB}$ is a soft ferromagnetic material widely used in tunneling magnetoresistence studies and commercial storage devices such as MRAM and HDD read heads. It is also a suitable material choice for magnetization dynamics experiments, in particular the amorphous (non-annealed) $\mathrm{Co}_{20} \mathrm{Fe}_{60} \mathrm{~B}_{20}$ or $\mathrm{Co}_{40} \mathrm{Fe}_{40} \mathrm{~B}_{20}$ alloy due to its high saturation magnetization $\left(\mu_{0} \mathrm{M}_{S}(\mathrm{CoFeB}) \approx 1.63 \mathrm{~T}\right)$. For comparison, the saturation magnetization of nickel ${ }^{74}$ is $\mu_{0} M_{S}(\mathrm{Ni})=0.613 \mathrm{~T}$ and yittrium iron garnet ${ }^{75}-\mu_{0} M_{S}(Y I G)=0.17 \mathrm{~T}$ at room temperature.

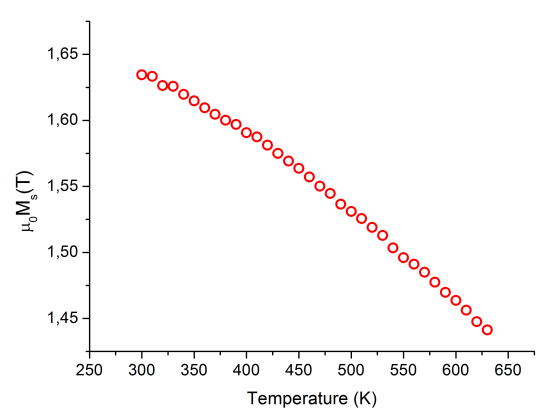

(a)

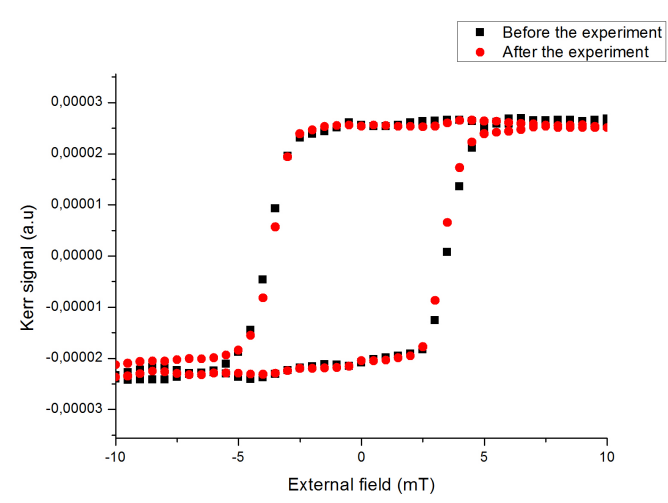

(b)

Figure 3.3: Magnetic properties of 50-nm CoFeB film. In a) vibrating sample magnetometry of saturation magnetization as function of temperature is shown. b) Hysteresis curves of in-plane magnetization before and after pulsed laser irradiation at $16 \frac{\mathrm{mJ}}{\mathrm{cm}^{2}}$ fluence.

Another interesting feature of $\mathrm{CoFeB}$ thin films is the uniaxial in-plane or out-of-plane magnetic anisotropy, that depends on parameters such as annealing conditions ${ }^{20}$ and film thickness $\frac{76}{}$, which origin is still debated. Since the films used in this work are at least $50 \mathrm{~nm}$ thick, all of them present an in-plane easy axis.

Thin (normally $50 \mathrm{~nm}$, unless noted otherwise) CoFeB films are magnetron sputtered on $\mathrm{Si}(100)$ or $\mathrm{SiO}_{2}$-thermally oxidized substrate and capped with ebeam deposited 2-nm Ru layer to prevent oxidation. The deposition process was carried out under ultrahigh vacuum conditions at room temperature.

Samples were characterized by measuring the saturation magnetization using a vibrating sample magnetometer (VSM) at 300-600 K and a hysteresis loop 
characterization using MOKE-setup at room temperature, before and after the irradiation with high-fluence laser beam.

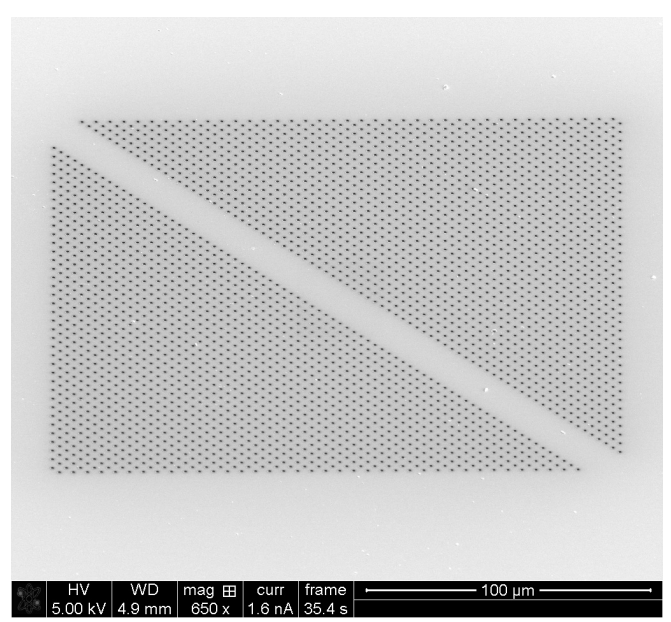

(a)

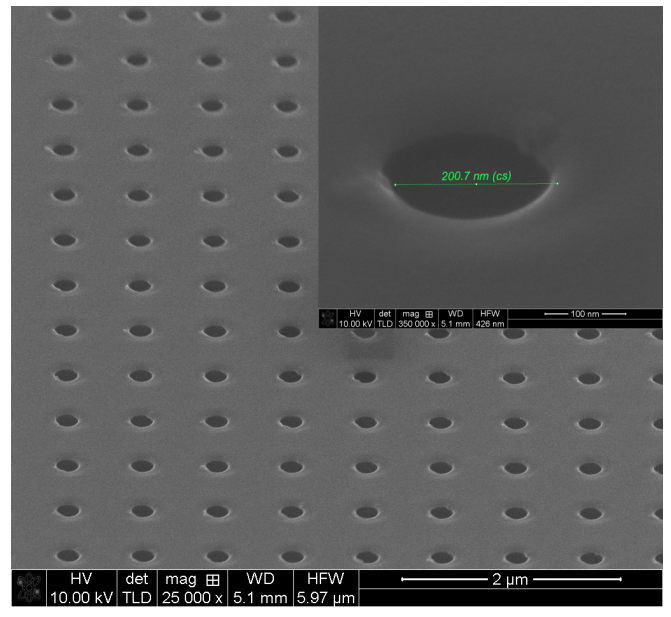

(c)

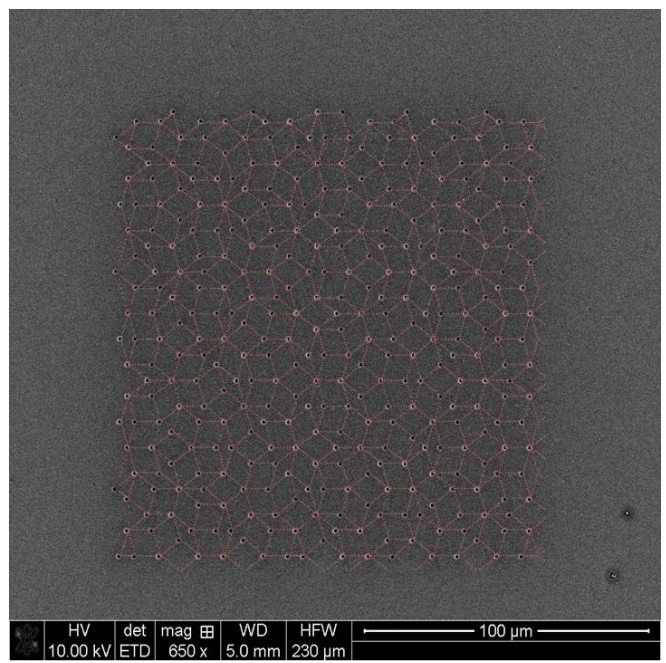

(b)

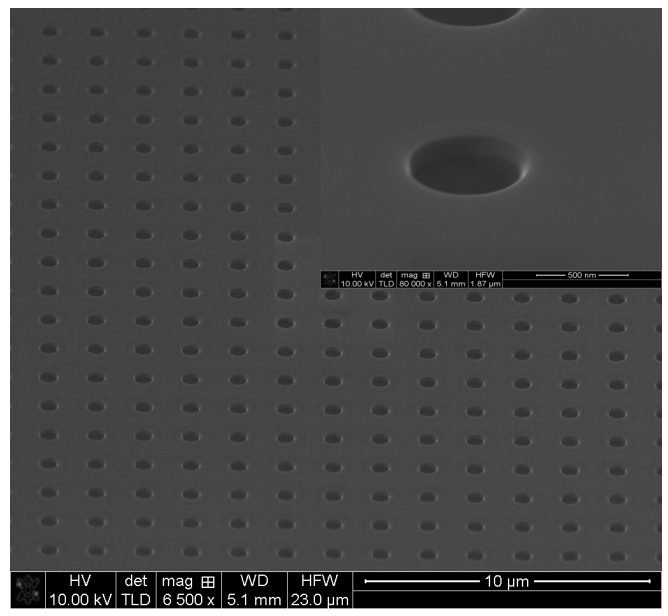

(d)

Figure 3.4: SEM images of structures with different periodicities and antidot sizes milled using FIB: a) defect line ( 5 missing rows) in a square antidot lattice $(\mathrm{a}=3.5 \mu \mathrm{m}, \mathrm{d}=1 \mu \mathrm{m})$ at $45^{\circ}$ to the lattice vector, $\left.\mathrm{b}\right)$ Penrose quasicrystal with $1 \mu \mathrm{m}$ diameter antidot and $a=3.5 \mu \mathrm{m}$ tessellation. Pink lines between antidots show the tessellation rhomboids. Maintaining a filling fraction of $\approx 6 \%$ structures with different lattice constant and antidot size can be milled: $a=700 \mathrm{~nm}, \mathrm{~d}=$ $200 \mathrm{~nm}(\mathrm{c})$ and $\mathrm{a}=1.75 \mu \mathrm{m}, \mathrm{d}=500 \mathrm{~nm}(\mathrm{~d})$. The insets in c) and d) show an individual dot in the corresponding lattice. 


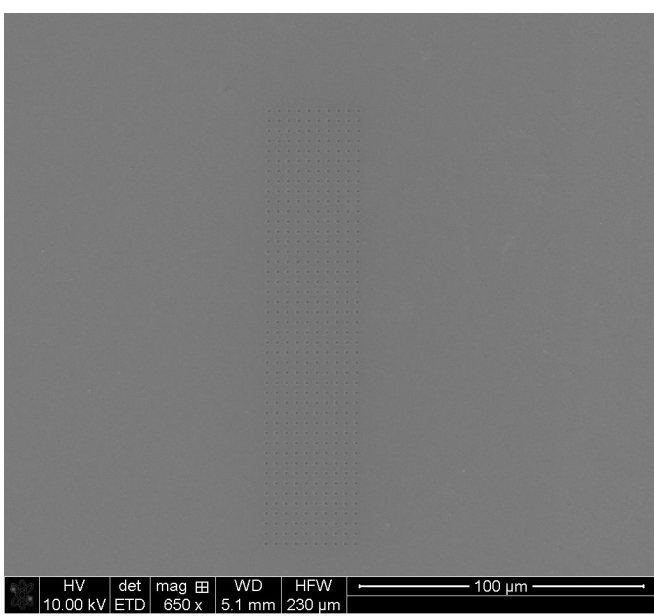

(a)

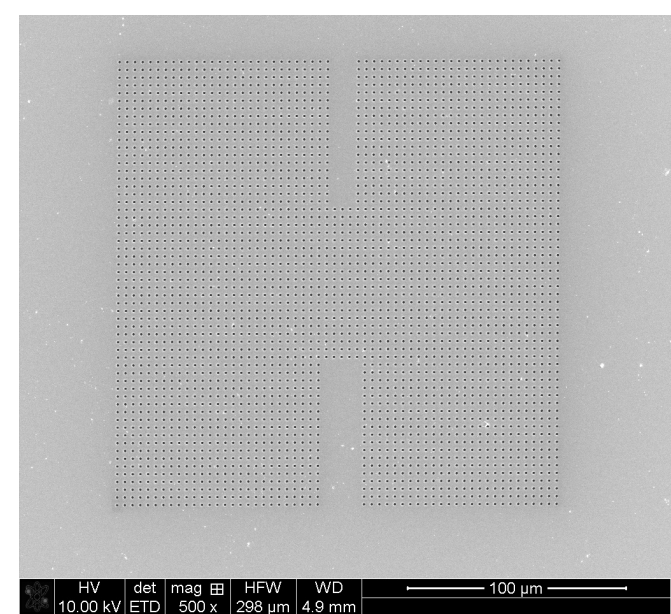

(b)

Figure 3.5: a) An inverted waveguide of 10 rows and b) a defect line of 5 rows parallel to the lattice vector in a $3.5 \mu \mathrm{m}$ square lattice of $1 \mu \mathrm{m}$ antidots. The pattern shown in b) was made by Benjamin Lenk ${ }^{77}$.

As can be seen in Fig. 3.3a, the saturation magnetization is temperature dependent (in accordance to Curie-Weiss law). However, although laser irradiation should cause an increase in sample temperature it does not change its magnetic properties, as the hysteresis curve before and after laser irradiation (see Fig. 3.3b) are identical. This indicates that the laser induced heating is dissipated before any annealing effect can take place.

After deposition, antidots were milled on the continuous CoFeB film in different geometries using FIB (FEI Nova NanoLab 600). This is a versatile, highperformance system that combines a high-energy $(30 \mathrm{kEv}) \mathrm{Ga}^{+}$focused ion beam and a $1.5 \mathrm{~nm}$ resolution scanning electron microscope (SEM), allowing pattern ion-milling and in-situ imaging 78 . The field of view on the sample is divided in $2^{16}$ bits (steps), that serve as coordinates for the positions of the ion beam. The sequence of $\mathrm{Ga}^{+}$beam positions requires an ASCII stream file, that contains the exact coordinates to which the ion gun is moved and milling times at each position. In order to produce the stream files for different patterns, a code in $\mathrm{C}$ programming language was developed. This allows producing practically any arbitrary array of antidots with diameter $d$ and lattice constant $a$. In particular, Fig. 3.4b shows a non-periodic Penrose quasicrystal. Periodic structures with line defects (waveguides), as shown in Fig.3.4a, and periodic arrays with different lateral sizes (for example Fig. 3.5a) were produced.

Antidots with a diameter as small as $200 \mathrm{~nm}$ can be milled using this technique. However, in this case the border of the antidot is not sharp, probably 
due to the fact that the film thickness is comparable to the antidot size. When milling larger diameter antidots (see for example Fig. 3.4d) sharp edges are easily obtained.

\subsubsection{Multilayer structures}

$\mathrm{W} / \mathrm{Si}, \mathrm{W} / \mathrm{Cu}$, and $\mathrm{W} / \mathrm{PC}$ (PC- polycarbonate) multilayered structures were grown by pulsed laser deposition (PLD), using a $\mathrm{KrF}$ excimer laser (248 nm wavelength, pulse duration $-30 \mathrm{~ns}$, and repetition rate $-10 \mathrm{~Hz}$ ) as described in Ref. 79. Further deposition details can be found in Ref. 80, Most materials grown by PLD present low surface roughness, even at larger than $100 \mathrm{~nm}$ film thicknesses, due to the smoothing effect ${ }^{81}$. For example, the intrinsic roughness of PC layers is $\approx 0.35 \mathrm{~nm}$, as determined by atomic force microscopy (AFM) measurements.

Metal/metal ( $\mathrm{FePtAgCu} / \mathrm{NiT})$ and metal/oxide $(\mathrm{CoFeB} / \mathrm{MgO})$ multilayers were grown by magnetron sputtering $(\mathrm{FePtAgCu}, \mathrm{NiTa}, \mathrm{CoFeB})$ and e-beam evaporation $(\mathrm{MgO})$.

In $\mathrm{CoFeB} / \mathrm{MgO}, \mathrm{PC} / \mathrm{Cu}$ and $\mathrm{W} / \mathrm{Si}$ multilayers, a good quality of growth was confirmed using transmission electron microscope (TEM) micrograph of multilayer cross-section (Fig. 3.6). Sharp interfaces between different layers are observed $^{80}$. Furthermore, in Fig. 3.6 is shown that $\mathrm{CoFeB}$ grows in an amorphous phase, while $\mathrm{MgO}$ grows with [001]-texture.

It can be concluded that we obtain thin layers of separate materials with sharp interfaces between the layers, that makes these high-quality samples adequate for multilayer studies. Moreover, some samples were deposited on polished glass substrates, due to its high transmittance at $800 \mathrm{~nm}$ wavelength, that allows for the pumping through the substrate.

\subsubsection{Bismuth telluride}

$\mathrm{Bi}_{2} \mathrm{Te}_{3}$ samples are epitaxially grown layers on $\mathrm{Si}$ substrates $(700 \mu \mathrm{m} \mathrm{Si}(001) / 0.3$ $\mu \mathrm{m} \mathrm{SiO}_{2} / 0.07 \mu \mathrm{m} \mathrm{Si} \mathrm{(111)).} \mathrm{Two} \mathrm{growth} \mathrm{domains} \mathrm{are} \mathrm{induced} \mathrm{by} \mathrm{the} \mathrm{substrate}$ (Fig 3.7), rotated $60^{\circ}$ with respect to each other.

These high quality samples were grown by molecular beam epitaxy at ultrahigh vacuum $\left(1 \times 10^{-9}\right.$ mbar base pressure). The system has $\mathrm{Bi}$, Te and $\mathrm{Sb}$ effusion cells giving the possibility of epitaxially growing $\mathrm{Bi}_{2} \mathrm{Te}_{3}$ compounds with different $\mathrm{Sb}$ dopings. However, typical growth rates are 5-20 $\frac{\mathrm{nm}}{\mathrm{h}}$, so that only thin $(\approx 15-30 \mathrm{~nm})$ continuous films are available. 


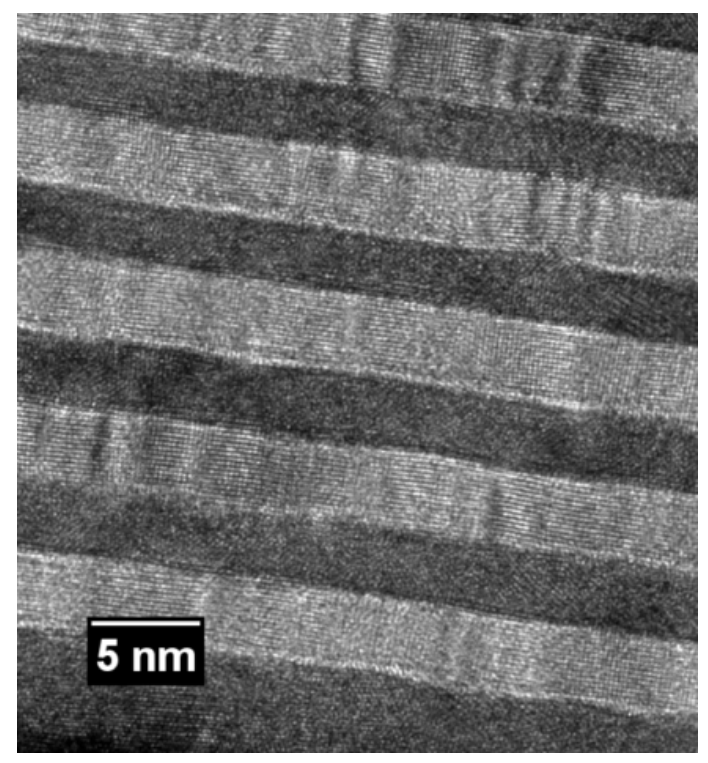

Figure 3.6: TEM images of $\mathrm{CoFeB} / \mathrm{MgO}$ multilayers in cross section. Image provided by Florian Döring.

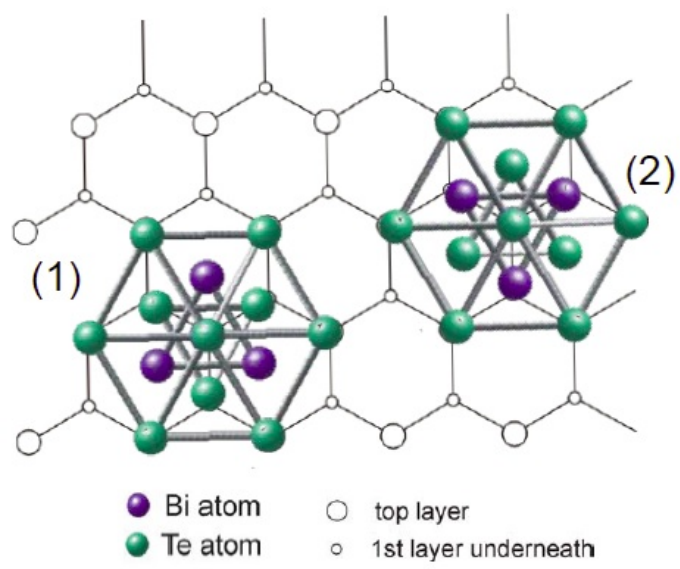

Figure 3.7: Relative orientation of $\mathrm{Bi}_{2} \mathrm{Te}_{3}$ unit cell on Si substrate. The two different growth domains are rotated $60^{\circ}$ with respect to each other. Image provided by Gregor Mussler. 


\section{Chapter 4}

\section{Magnetization dynamics in sub-micron antidot lattices on thin CoFeB films}

In the following chapter, we discuss the main experimental results concerning the characteristics of thermally excited magnetization dynamics in a magnonic crystal, as well as on different topologies such as the interface between magnonic crystal and continuous ferromagnetic film, and defect lines. The main focus of the chapter is magnetization dynamics on sub-micron antidot lattices and the emergence of localized spin wave modes. The richness of the excited spin-wave spectrum and the possibility of tuning spin-wave frequency using several parameters, such as film thickness and antidot separation, allows us to address cases of special interest, namely the coexistence of different spin-wave modes with the same frequency in a magnonic crystal and its effects on Gilbert damping. Moreover, spatially-resolved magnetization dynamics measurements and the availability of a high-fluence laser, allow to address also the effects of laser heating on the frequencies and dispersion characteristics of spin waves.

Magnetization and reflectivity dynamics experiments reported in this chapter were done in collaboration with Frederik Busse, Judith von der Haar, and Stefan Behrens. Data analysis and interpretation was done in collaboration with Markus Münzenberg and Henning Ulrichs. Static magnetization simulations in $\operatorname{mumax}^{82}$ and the simulations of the laser heating of thin films in COMSOL were performed by Henning Ulrichs and Marvin von der Ehe, respectively. 


\subsection{General spin-wave properties: From continuous CoFeB thin films to a magnonic crystal}

In this section spin-wave properties of continuous and nano-patterned thin $\mathrm{CoFeB}$ films are discussed. First, local magnetization dynamics properties, such as the dependence of spin-wave frequency on external magnetic field are determined, allowing to identify the excited spin-wave modes (uniform precession, perpendicular standing spin wave or Damon-Eshbach-like mode) via the characteristic spin-wave dispersion (eqs. (2.7) to (2.10)). Later on, spatially-resolved measurements of spin-wave dynamics on different geometries, such as the interface between a magnonic crystal and continuous film, and defect lines in a magnonic crystal are discussed. This provides some information about the emergence of magnonic modes and gives a hint on the effective field distribution in the magnonic crystal.

In order to saturate the $\mathrm{CoFeB}$ thin film and allow the spin-wave excitation, an external magnetic field is applied at some angle to the sample plane (typically $20-30^{\circ}$ ). Since a strong in-plane anisotropy field is always present in such films, as previously discussed in section 2.1.1, the resulting magnetization direction will be canted $2-3^{\circ}$ out of the sample plane. The importance of this slight out-of-plane canting can be found in section 2.3.2. Additionally, this enables a longitudinal MOKE detection scheme, as the magnetization vector will now

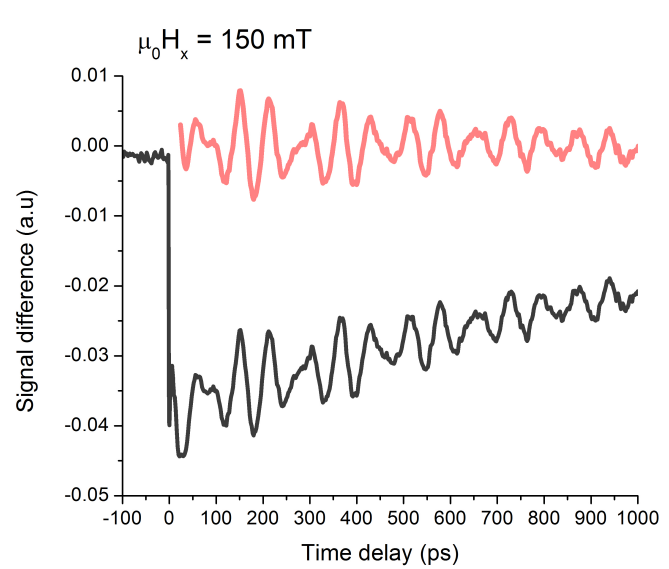

(a)

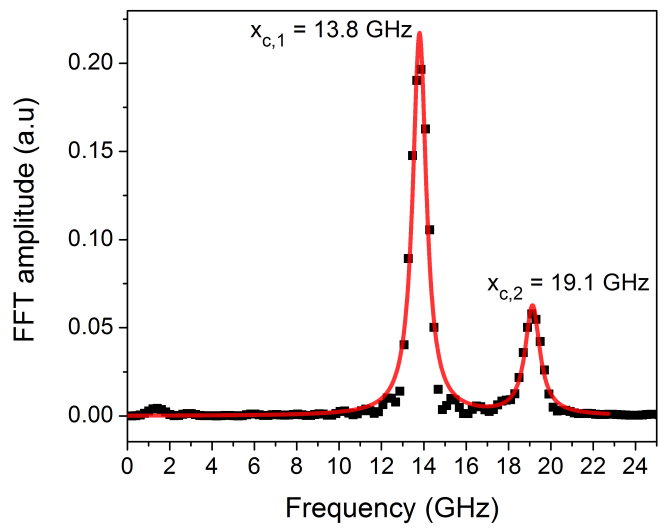

(b)

Figure 4.1: a) MOKE signal difference at $150 \mathrm{mT}$ external magnetic field as function of pump-probe time delay (in black) and the same signal after incoherent (linear) background subtraction (red). b) FFT amplitude of autocorrelated signal. Two main frequencies are visible. Red line is a sum of Lorentzian peaks fit to the data with central frequencies $x_{c, 1}=13.84 \mathrm{GHz}$ and $x_{c, 2}=19.1 \mathrm{GHz}$. 
have not only a time-dependent polar component, but also a small longitudinal one. The details of MOKE detection scheme and the relation between the Kerr angle and the time-dependent magnetization can be found in section 3.2.2.

A typical MOKE difference signal (between antiparallel directions of external magnetic field) on continuous $50 \mathrm{~nm}$ CoFeB film is shown in Fig 4.1a. A rapid demagnetization is observed in the first picoseconds after the arrival of the pump pulse and then a slow remagnetization back to the equilibrium value for up to $1 \mathrm{~ns}$. Also, a coherent magnetization oscillation is launched and can be observed additionally to the incoherent linear background. As part of data evaluation process, this incoherent background is subtracted leaving a clear oscillation with a beating pattern, due to the presence of several fundamental frequencies in the signal. To determine these frequencies, the autocorrelated MOKE signal is fast Fourier-transformed (FFT), as shown in Fig. 4.1b.

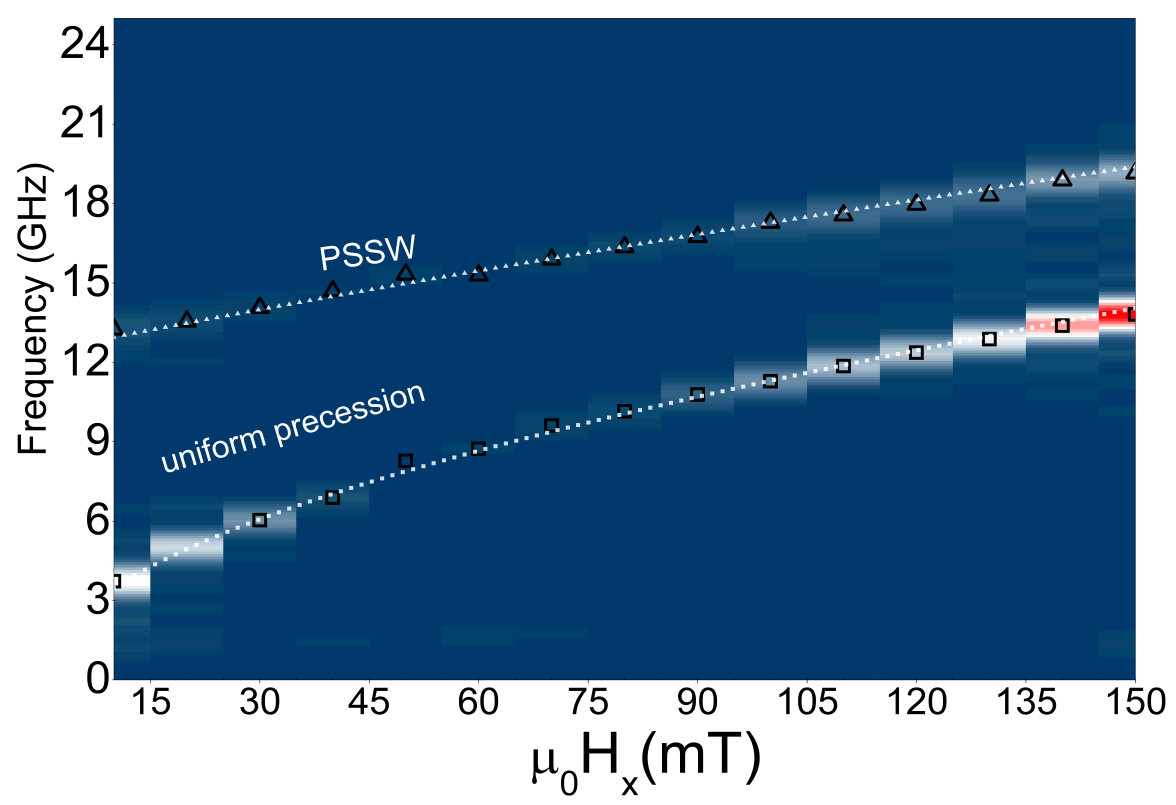

Figure 4.2: FFT amplitude (in color code) of autocorrelated MOKE signal on a continuous 50-nm CoFeB film. Two modes are visible, that can be identified as uniform precession (Kittel mode) and PSSW. Frequency peaks are marked in squares and triangles respectively. White dotted lines are numerical fits to the data.

When the spin-wave frequencies are measured as function of the applied magnetic field, we can determine which spin-wave modes are excited in the continuous film (Fig. 4.2) by fitting the appropriate spin-wave dispersion relation. This allows to identify the Kittel and PSSW modes $\frac{83}{\text {. Magnetization }}$ 
dynamics on antidot lattices shows an additional, magnonic Damon-Eshbachlike mode. This mode extends through the lattice in the channels contained between antidot rows normal to the applied magnetic field, i.e in Damon-Eshbach geometry, with a wave vector $\vec{k} \perp \vec{H}_{\text {eff }}$. Moreover, the wave vector magnitude is determined by the lattice constant $a,|k|=\frac{\pi}{a}$ as shown in a previous work 30 . Due to these characteristics, this magnonic mode is often called Damon-Eshbach mode in literature.

\subsubsection{Interface between a magnonic crystal and continuous film}

An interesting question that arises when addressing magnonic crystals, is the role played by the interface between the continuous film and the antidot lattice and how the modification of internal field influences the spin wave frequencies. Spatially-resolved magnetization dynamics measurements were performed by scanning the overlapped (on the center) pump and probe spots across the sample, in particular across the interface between an antidot lattice and continuous $\mathrm{CoFeB}$ film, at a constant external magnetic field. The spatial scanning step size amounts to $8.5 \mu \mathrm{m}$, while the Gaussian pump and probe FWHM are $60 \mu \mathrm{m}$ and $28 \mu \mathrm{m}$ respectively. The antidot lattice with a lattice constant $\mathrm{a}=3.5 \mu \mathrm{m}$ and antidot diameter $\mathrm{d}=1 \mu \mathrm{m}$ was milled on a $50-\mathrm{nm} \mathrm{CoFeB}$ film as described in section 3.3.1.

The external magnetic field $\left(\mu_{0} \mathrm{H}=130 \mathrm{mT}\right)$ was applied at $45^{\circ}$ to the antidot lattice vector. The scanning direction of spatially-resolved measurements also lies at $45^{\circ}$ to the lattice vector and the interface between the antidot lattice and continuous film, as schematically shown in top inset of Fig. 4.3b. The FFT amplitude of magnetization dynamics (in color code) at a given frequency, as function of the position of the overlapped pump and probe spots on the sample, is shown in Fig. 4.3a. The spin-wave modes can be identified by comparing the excited frequencies to the field-dependent magnetization dynamics measurements in Fig. 4.2. The interface between the magnonic crystal and the continuous field can be identified by measuring the spatial variation of reflected laser intensity $\frac{\mathrm{dR}}{\mathrm{dx}}$. As the laser beam crosses the interface the reflected intensity variation will be maximum (upper panel in Fig. 4.3a).

As can be seen, on continuous film both Kittel and PSSW modes are found, although Kittel mode dominates the spin-wave spectrum due to its large amplitude. This can be expected, since the Kittel mode is a lower-order quantized spin wave mode $(n=0)$ than the PSSW $(n=1)$. When approaching the magnonic crystal interface, the amplitude of Kittel mode is strongly decreasing, and finally on the magnonic crystal the PSSW and DE mode have the largest amplitude, while the Kittel mode amplitude is strongly suppressed in comparison. In 
general, as can be seen in Fig. 4.3b, the spin-wave amplitude on the continuous film is about 10 times larger than on the magnonic crystal. One of the reasons for this is that the magnonic crystal reflects less laser intensity due to the milling of antidots $(\approx 6 \%$ less reflecting material). Also, the laser beam reflects on the magnonic crystal in a diffraction pattern (because the laser wavelength is comparable to the distance between antidots) and only the first-order diffraction spot goes through the detection optics: i.e., the light intensity on the detection diode is decreased and, in general, the detection of spin waves on the antidot lattice is more difficult.

However, more important that the removal of reflecting material and the loss of signal intensity, is the loss of uniformity of internal field due to the demagnetizing fields induced by the antidots. While on the continuous film the Kittel mode can extend practically over the whole film area, on the antidot lattice spinwave channels are formed. In this particular configuration, with external field at $45^{\circ}$ to the lattice vector, the spin-wave channels lie along the symmetry directions (lattice vectors) and contain the DE mode in the areas of uniform internal field between antidots. This way, less film area with uniform internal field is available to the different spin-wave modes, compared to the continuous film. Thus, a reduction in mode amplitude is also expected.

On the other hand, in Fig. 4.3a we can see that, although the Kittel mode frequency is practically constant on the continuous film, it is reduced by $\approx$ $0.62 \mathrm{GHz}$ or $4.8 \%$, on the magnonic crystal. In contrast, the shift of PSSW frequency is practically negligible over the whole range of the measurement, both on continuous film and magnonic crystal, with an average frequency increase of $0.06 \mathrm{GHZ}$ on the magnonic crystal.

The decrease of Kittel frequency on the magnonic crystal, compared to the continuous film, can be explained in terms of a reduction of the effective magnetic field in the magnonic crystal, as a consequence of the dipolar fields created by each antidot. The change in Kittel frequency as function of effective field variation can be calculated by taking the derivative $\frac{d_{\omega_{K i t t e l}}}{d H_{\text {eff }}}$. As we can see from Eq. 2.8, the Kittel mode frequency also depends on the saturation magnetization, which is a material parameter and can be regarded as constant in this case. This way, we calculate:

$$
\begin{aligned}
\mu_{0} \Delta \mathrm{H}_{\text {eff }} & =\frac{2 \omega_{\text {Kittel }}}{\gamma\left(2 \omega_{\mathrm{H}}+\omega_{\mathrm{M}}\right)} \Delta \omega_{\text {Kittel }} \approx 5.5 \mathrm{mT} \\
\frac{\Delta \mathrm{H}_{\text {eff }}}{\mathrm{H}_{\text {eff }}} & =4.5 \%
\end{aligned}
$$

The shift in Kittel mode frequency could be caused by a $4.5 \%$ average reduction in the internal magnetic field. 


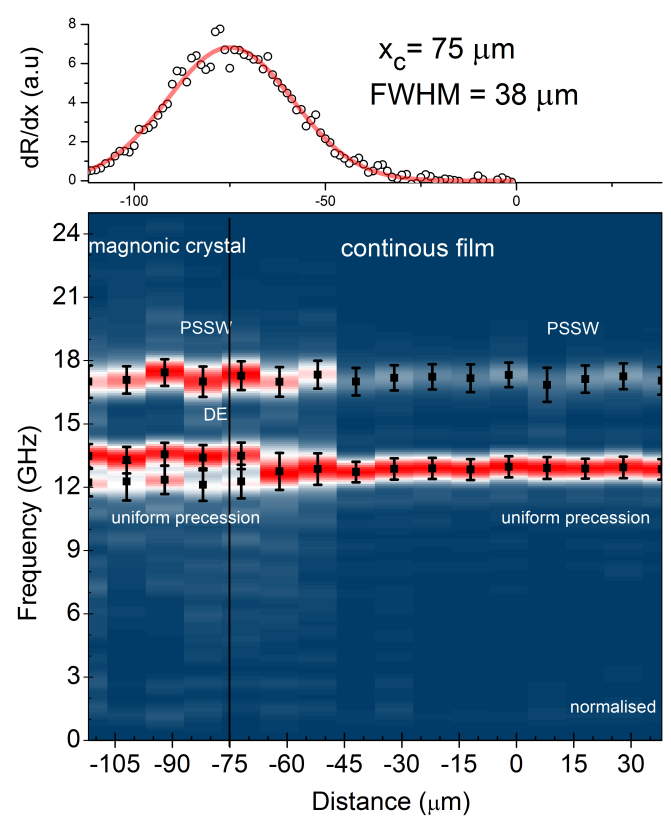

(a)

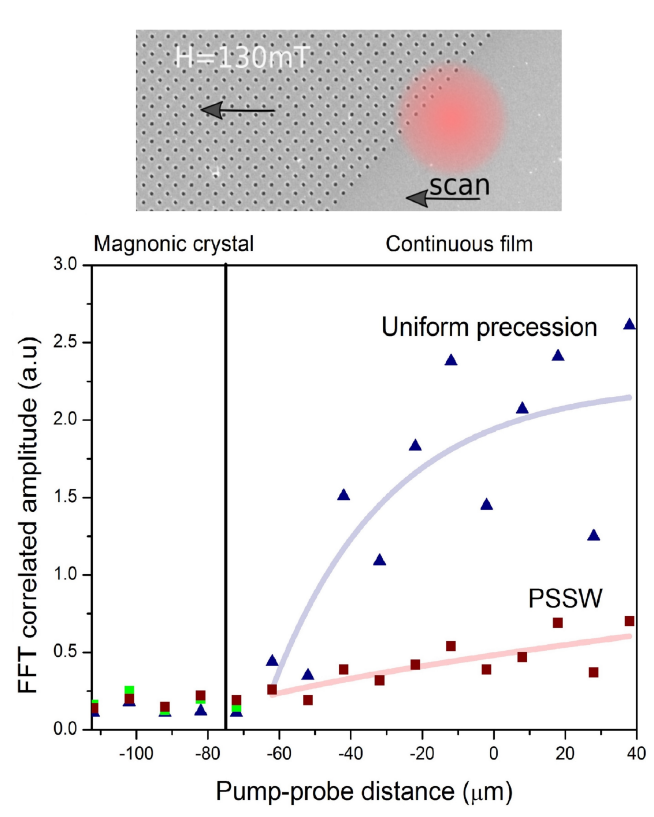

(b)

Figure 4.3: a) Normalized FFT amplitude (in color code) of the magnetization oscillations when scanning pump and probe beams (overlapped) over the sample. Black line indicates the interface between the magnonic crystal $(a=3.5 \mu \mathrm{m}$ , $\mathrm{d}=1 \mu \mathrm{m}$ ) and continuous $50 \mathrm{~nm}$ CoFeB film at $75 \mu \mathrm{m}$. Top inset shows the change in reflected probe intensity as function of displacement. Red curve is a Gaussian fit with central position $x_{c}=75 \mu \mathrm{m}$ and a $38 \mu \mathrm{m}$ FWHM. Three modes are visible: uniform precession (Kittel mode), PSSW and DE mode. b) FFT amplitude of correlated signal on continuous film and magnonic crystal. Lines are guides to the eye. Top inset shows a schematics of the relative orientations of the magnonic crystal, the overlapped pump and probe scan direction and the applied magnetic field.

On the other hand, the PSSW mode depends also on the effective field, however the functional dependence is quite different. As can be seen in Eq. 2.11. for first-order PSSW mode $(n=1)$, an additional contribution to the frequency exists, namely $\omega_{A}$. This term shows the exchange character of the PSSW mode; it includes the exchange constant $A$, which is a material parameter and is expected to be the same on the magnonic crystal an the continuous film, at least at first approximation. Moreover, it can be assumed that the sample thickness is constant $d=50 \mathrm{~nm}$ in the scanned area. Proceeding in the same manner as 
for the Kittel mode, we can calculate:

$$
\Delta \omega_{\mathrm{PSSW}}=\frac{\gamma\left(2 \omega_{\mathrm{H}}+2 \omega_{\mathrm{A}}+\omega_{M}\right)}{2 \omega_{\mathrm{PSSW}}} \mu_{0} \Delta \mathrm{H}_{\text {eff }} .
$$

The pre-factor in the equation above is strictly positive, so that only an increase in effective field would yield an increased frequency on the antidot lattice. The minimal observed shift is $0.06 \mathrm{GH} z$, equivalent to $\Delta \mathrm{H}_{\text {eff }} \approx 1 \mathrm{mT}$ or a $0.8 \%$ increase in effective field.

Ignoring the second order terms in $\omega_{\text {Kittel }}$ and $\omega_{\text {PSSW }}$ we can estimate:

$$
\begin{gathered}
\frac{\Delta \omega_{\text {Kittel }}}{\omega_{\text {Kittel }}}=\frac{1}{2} \frac{\gamma \Delta \mathrm{H}_{\text {eff }}}{\omega_{H}}, \\
\frac{\Delta \omega_{\text {PSSW }}}{\omega_{\text {PSSW }}}=\frac{1}{2} \frac{\gamma \Delta H_{\text {eff }}}{\omega_{H}+\omega_{A}},
\end{gathered}
$$

so that, PSSW mode frequency is only slightly less sensitive to a variation of effective magnetic field. Moreover, small fluctuations in intensity and frequency of the modes (see figs. $4.3 \mathrm{a}$ and $4.3 \mathrm{~b}$ ) might be due to small defects or irregularities on film surface.

\subsubsection{Magnetization dynamics on defect lines in a magnonic crystal}

In this section field-dependent magnetization dynamics in a waveguide, as well as spatially-resolved magnetization dynamics along the waveguide at constant magnetic field are carried out. A waveguide is a defect line in the magnonic crystal tat consists of missing rows of antidots, creating a broad continuous film region inside the magnonic crystal.

The width of the waveguide, defined as the width of continuous film between rows of antidots on each side of the defect, amounts to $20 \mu \mathrm{m}$, equivalent to 5 missing rows of antidots. On the other hand, the pump and probe beam FWHM amount to $94 \mu \mathrm{m}$ and $24 \mu \mathrm{m}$, respectively. The probe spot was centered on the waveguide, so that the probe FWHM extends over the waveguide plus a unit cell length in total. This means that most of the signal intensity carried by the probe beam comes from the waveguide. Moreover, since the pump spot is much larger than the waveguide length, we can suppose that the excitation is fairly uniform over the waveguide.

The magnetization dynamics properties of a waveguide are, in some cases, quite different from those of a simple continuous film. Although DE modes are not found in the continuous $\mathrm{CoFeB}$ film but only on the magnonic crystal, they 


\section{CHAPTER 4. MAGNETIZATION DYNAMICS IN SUB-MICRON ANTIDOT LATTICES ON THIN COFEB FILMS}

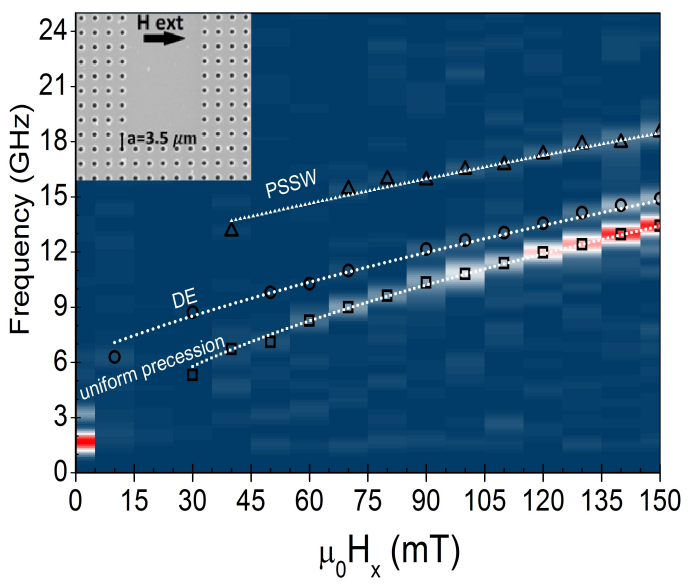

(a)

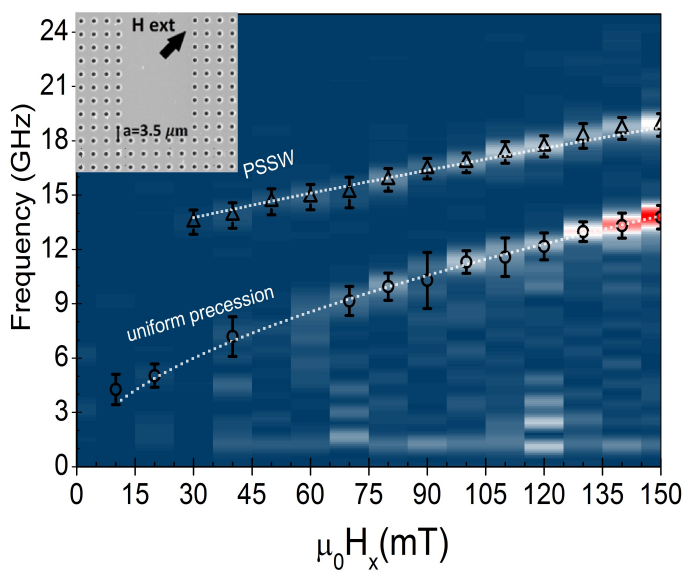

(b)

Figure 4.4: FFT amplitude (in color code) of magnetization oscillations in the middle point of a $20 \mu \mathrm{m}$ (or 5 removed rows) waveguide in a $(a=3.5 \mu \mathrm{m}, \mathrm{d}=$ $1 \mu \mathrm{m})$ magnonic crystal. Insets show the relative orientation of the waveguide a) $90^{\circ}$ and b) $45^{\circ}$ to the external magnetic fields. Same modes as on the magnonic crystal are observed: Kittel (squares), DE (circles) and PSSW (triangles) modes.

are clearly detected in the waveguide when the external field is applied at $90^{\circ}$ to the longitudinal axis of the waveguide, as shown in Fig. 4.4a. Moreover, the corresponding DE wave vector is $k=\pi / a$, matching that on the magnonic crystal, and the amplitude of the DE mode is comparable to the one detected on the magnonic crystal.

On the other hand, when the external magnetic field is applied at $45^{\circ}$ to the waveguide longitudinal axis, no DE mode is detected, but only Kittel and PSSW modes, as on continuous film. This can be clearly seen in the low-field magnetization dynamics in Fig. 4.4b, where the detected mode clearly follows the Kittel dispersion relation.

As next step, spatially-resolved measurements along the waveguide longitudinal axis were carried out. The pump and probe were initially overlapped at the entrance to the waveguide $(0 \mu \mathrm{m}$ position in the schematics shown in Fig. 4.5b). Then, the pump was displaced along the waveguide axis scanning the region surrounded by magnonic crystal (positive direction) and the continuous film (negative direction), while the probe was kept at fixed position. As can be seen in Fig. 4.5a, the Kittel and PSSW modes amplitude follows a Gaussian profile, decaying with increasing pump-probe distance. Moreover, local demagnetization can be estimated from the amplitude of the initial fast demagnetization peak in the time-resolved signal (see for example Fig. 4.1a) for each 


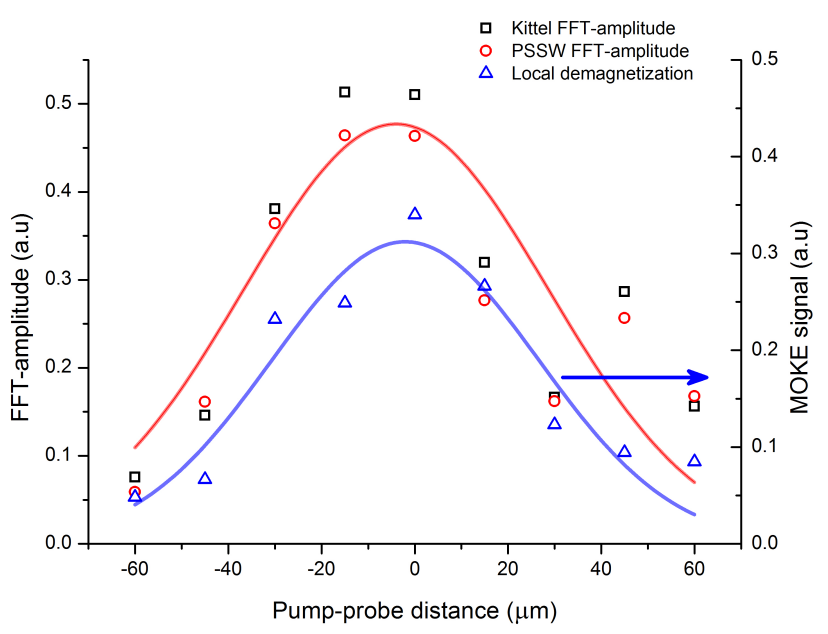

(a)

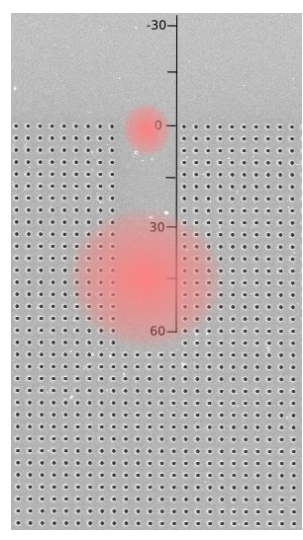

(b)

Figure 4.5: a) The amplitude of demagnetization peak (blue triangles), FFT of Kittel (black squares), and PSSW (red circles) modes in a magnonic waveguide at $45^{\circ}$ to the external magnetic field as function of pump position along the waveguide. Solid lines are Gaussian fits to each data set. b) Schematics of the relative displacement of pump and probe in the waveguide. Probe spot (smaller red circle) remains at the entrance to the waveguide $(0 \mu \mathrm{m})$ while the pump spot (large red circle) is scanned across the waveguide in positive (into the waveguide) and negative (towards the continuous film area) directions.

position of the pump spot: as can be seen in Fig. 4.5a it also follows a Gaussian distribution. This can be understood in terms of the Gaussian pump spot intensity profile i.e., it can be expected that higher intensity in the center of the pump spot induces a larger initial demagnetization that would excite magnetization oscillations with a higher amplitude. Thus, no additional features of spin-wave modes, that could be attributed to the magnonic waveguide in comparison with the continuous film, were observed.

\subsubsection{Discussion}

As we have seen in this introductory section, the milling of an antidot lattice largely influences the frequency of excited spin wave modes via the modification of the internal magnetic field in the lattice. In particular, the frequency of Kittel mode on the antidot lattice is reduced by a larger amount than the PSSW, that could not be explained by a different frequency dependence on effective field variations. This suggest that, although Kittel and PSSW modes vary only in the order of the bulk spin-wave mode, they might be localized in different 
regions of effective field in the antidot lattice. In particular, the negligible frequency shift of PSSW mode in the antidot lattice, compared to continuous film, suggests that it is localized on regions with less than $1 \%$ variation of the internal field compared to the external field. In contrast, the Kittel mode is localized in the strongly $(5 \%)$ varying regions of the effective field, probably closer to the antidots, i.e., an edge mode. Another argument in this direction is the low amplitude of the Kittel mode as compared to PSSW and DE modes on the antidot lattice. When the external field is applied at $45^{\circ}$ to the lattice vector, two spin wave channels are formed along the same direction with a large area of uniform, slightly reduced, internal field where DE mode is extended. Thus, if the PSSW mode is also localized in regions with only slightly reduced internal field, they should have a comparable signal amplitude, as in fact they do (see Fig. 4.3a).

Removing 5 rows of the magnonic crystal restores the internal field practically to that of the continuous film, since no DE mode was detected in the waveguide at $45^{\circ}$ to the external magnetic field. It must be noted that the twofold symmetry of spin-wave channels where DE mode extends is broken around the waveguide when the field is tilted at $45^{\circ}$. However, when the external field is directed along the antidot lattice vector, $\mathrm{DE}$ mode extends only in channels lying at $90^{\circ}$ to the external field, i.e., parallel to the longitudinal axis of the waveguide. Thus, it might be expected to find a DE mode only in this configuration, if the probe spot size is small enough.

\subsection{Coexistence of perpendicular standing spin wave and Damon-Eshbach spin waves in a magnonic crystal}

As can be already seen from the Eq. 2.9 and 2.11, both DE and PSSW modes depend not only on the applied external magnetic field, but also on the film thickness. This means that these parameters can be tuned in such a way that PSSW and DE modes have the same frequency at some given external magnetic field.

Using the dispersion relations, the dependence of spin-wave frequency on film thickness was plotted for DE and PSSW modes in Fig.4.6. As can be noted, two possibilities for excitation of same frequency of PSSW and DE modes at $100 \mathrm{mT}$ are available: if the lattice constant is kept at $3.5 \mu \mathrm{m}$, then the film thickness should be increased to approximately $82.5 \mathrm{~nm}$; on the contrary, if the film thickness was kept at $50 \mathrm{~nm}$, then the lattice constant must be reduced to $1.75 \mu \mathrm{m}$. In summary, either the thickness of ferromagnetic film should be 


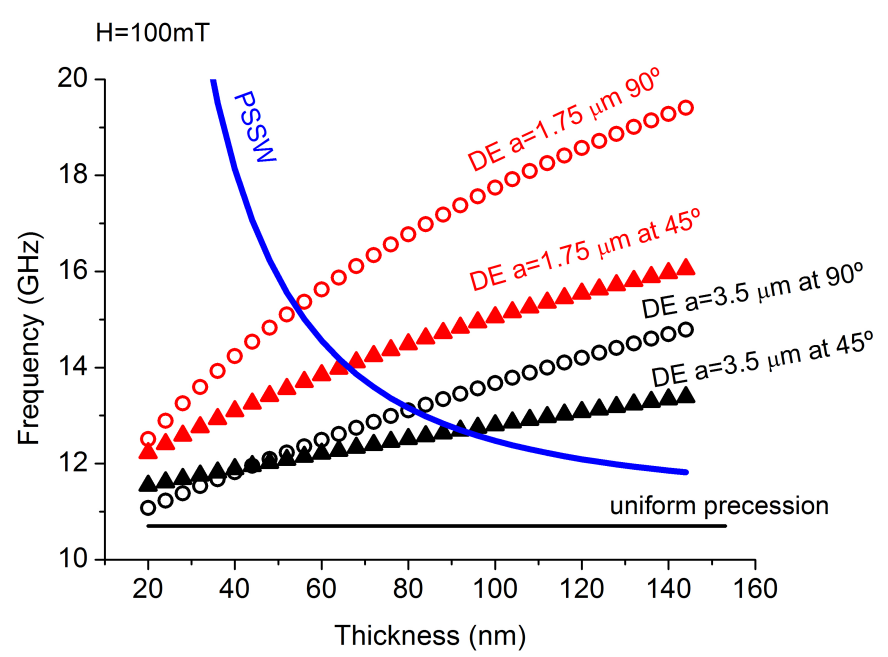

Figure 4.6: The dependence of DE (for $1.75 \mu \mathrm{m}$ (in red) and $3.5 \mu \mathrm{m}$ (in black) lattice constant) and PSSW (in blue) frequencies on the thickness of CoFeB film at $100 \mathrm{mT}$ external magnetic field. Uniform precession frequency is shown as a constant line. DE frequencies for perpendicular (circles) and $45^{\circ}$ (triangles) orientation of external magnetic field with respect to the magnonic crystal highsymmetry directions are shown.

increased or the lattice constant must be reduced. Both these options present some challenges when milling the magnonic crystal, as discussed in Methods chapter. In this section, only magnetization dynamics on 82.5-nm thick film are discussed. The second case $(1.75 \mu \mathrm{m}$ antidot lattice on $50 \mathrm{~nm}$ film) is discussed in the next section, because the excited magnetization dynamics on this film is radically different.

When increasing CoFeB film thickness, an additional high-frequency spinwave mode is excited on the continuous film as shown in Fig 4.7a. This additional mode can be identified as a second order $(n=2)$ PSSW, whose excitation is due to the increased thickness of the film as compared to the optical penetration depth of laser light into the sample $(\sim 22 \mathrm{~nm} \text { for } \mathrm{CoFeB})^{77}$.

The uniform precession frequency is not changed by increasing thickness, as must be expected from its dispersion relation (Eq. 2.8). More importantly, the Gilbert damping constant calculated using Eq. 2.12 and the spin-wave decay time (determined by fitting an exponentially modulated sine wave to the experimental data in the time domain), remains very close to the value of the damping constant in a 50-nm CoFeB film of slightly different composition $(\alpha=0.00646 \pm 0.00022$ as shown in Fig. 4.7b). Note that the Gilbert damping is also left unchanged by the external magnetic field. This allows us to conclude that the damping of spin waves excited on a magnonic crystal milled on such 


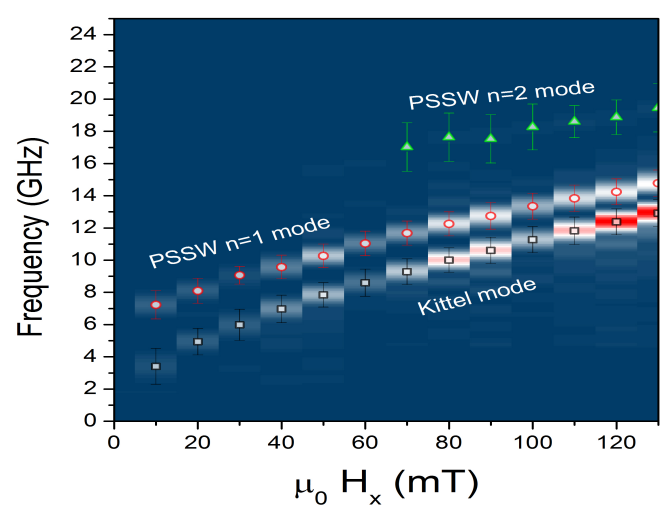

(a)

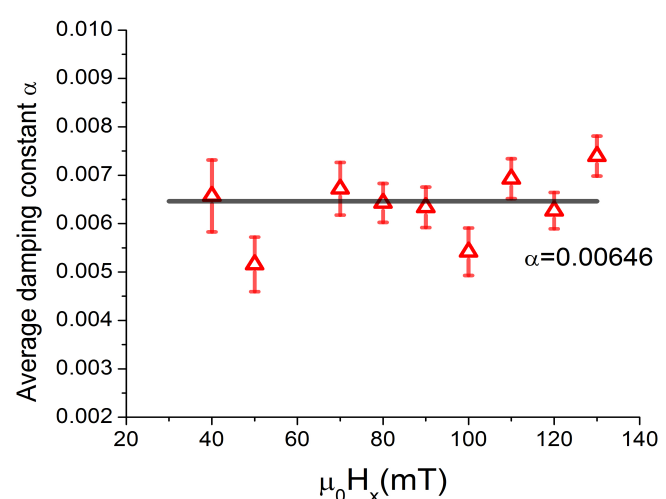

(b)

Figure 4.7: a) FFT amplitude (in color code) of magnetization precession on a continuous 82.5-nm CoFeB film. Three modes are observed: Kittel mode, first $(n=1)$ and a high-frequency second-order $(n=2)$ PSSW modes. The central frequencies of Gaussian fits to the amplitude peaks are marked in squares (Kittel mode), circles (PSSW $n=1$ mode) and triangles (PSSW $n=2$ mode). b) Average Gilbert damping of Kittel and PSSW mode in continuous CoFeB film as function of applied external field. Black line indicates a linear fit to the data $\alpha=0.00646 \pm 0.00022$

film should be no worse than that on thinner films.

The results of magnetization dynamics measurements on the magnonic crystal with the external magnetic field oriented along the antidot lattice vector, are shown in Fig. 4.8a. Beside the same spin-wave modes as on the continuous film (including second order PSSW mode) an additional DE mode $(k=\pi / a)$ is observed at low external magnetic fields (see Fig. 4.8b for better visibility). At $80 \mathrm{mT}$ and larger fields, the amplitude peaks corresponding to DE and PSSW modes become indistinguishable, as expected from the previous frequency calculations shown in Fig. 4.6

Although a modification of the dispersion or frequency characteristics of DE and PSSW is not expected, due to their different wave vectors, the Gilbert damping in the magnonic crystal is increased (by a factor of 2) compared to the damping of spin waves on continuous films. As can be seen in Fig. 4.9a, the damping of uniform precession increases with increasing external field, from a value close to that of continuous film at low fields. In contrast, the damping of the PSSW decreases with the external magnetic field, and both modes converge to $\alpha \approx 0.0125$ at high magnetic fields. Consequently, the amplitude of merged DE and PSSW modes is larger than the uniform precession amplitude, as shown in Fig. 4.9b. This is in contrast to the magnetization dynamics on a 


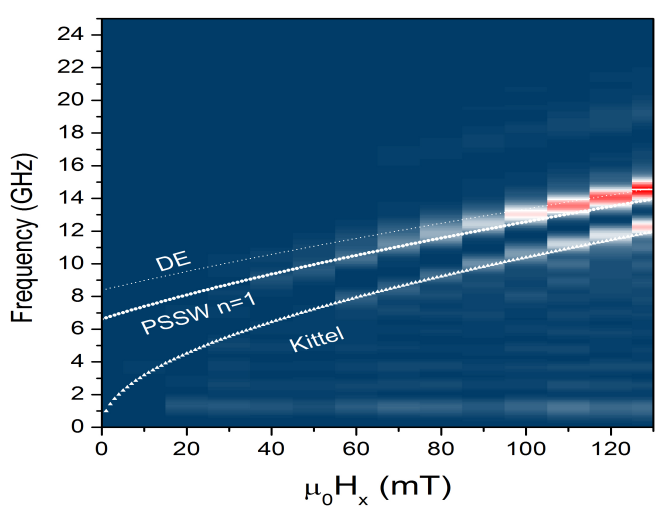

(a)

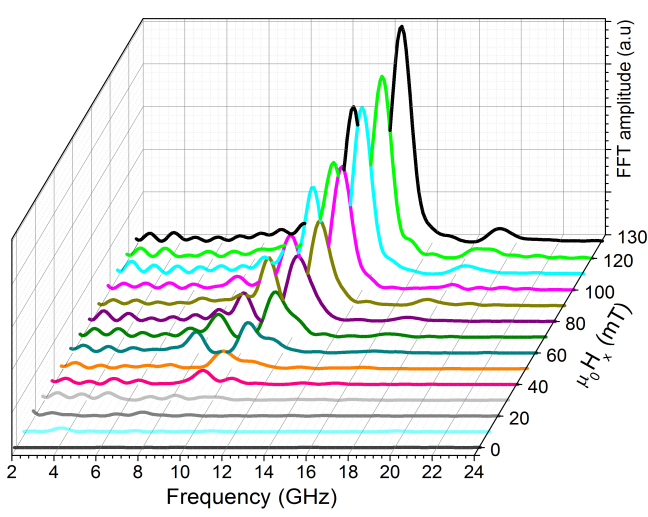

(b)

Figure 4.8: a) FFT amplitude (in color code) of magnetization precession in a magnonic crystal $(a=3.5 \mathrm{mum}, \mathrm{d}=1 \mu \mathrm{m})$ on $82.5-\mathrm{nm}$ CoFeB film. Lines indicate the expected theoretical Kittel, PSSW $(n=1)$ and DE mode frequencies. Second-order PSSW mode is seen at higher frequencies (not marked). Same data is plotted in b) in real FFT amplitude for better visibility. A merging of DE and PSSW modes can be observed at $60 \mathrm{mT}$ external magnetic field.

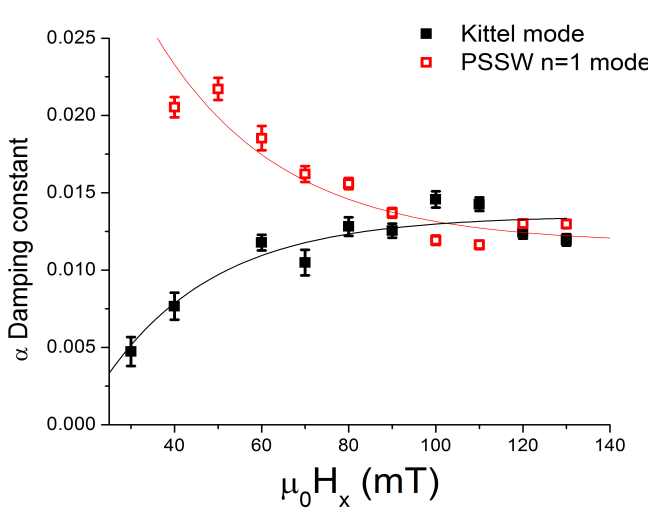

(a)

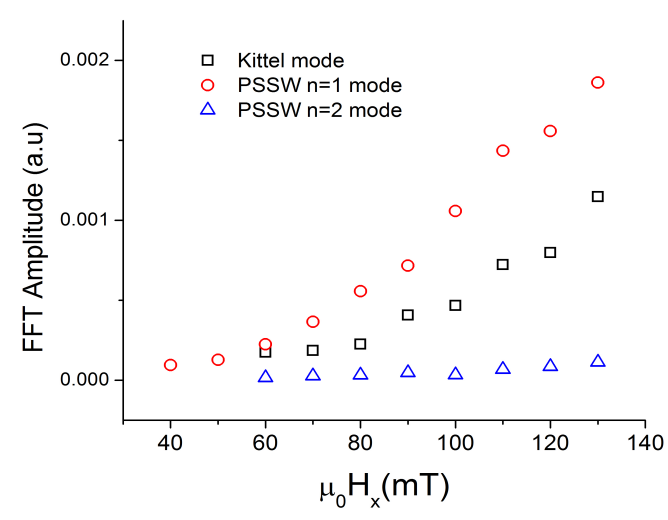

(b)

Figure 4.9: a) Gilbert damping of Kittel (solid squares) and PSSW modes (hollow squares) in a magnonic crystal $(a=3.5 \mu \mathrm{m}, \mathrm{d}=1 \mu \mathrm{m})$ on 82.5-nm CoFeB film. External magnetic field points along the antidot lattice vector. Solid lines are a guide to the eye. b) FFT amplitudes of magnetization oscillation modes in the magnonic crystal.

continuous film, where uniform precession amplitude dominates the spectrum at high external magnetic fields as indicated by the red color in Fig. 4.7a. 


\subsection{Sub-micron magnonic crystals}

In this section, magnetization dynamics on a sub-micron antidot lattice milled on a 50-nm CoFeB film is discussed. As we will see later, also the elastic properties of such films and of magnonic crystals on them must be addressed, because of a possible interplay between elastic and magnetic properties.

\subsubsection{Elastic dynamics}

As shown in Fig. 4.10a, a laser pulse incident on the continuous film excites fast elastic dynamics in the first $100 \mathrm{ps}$ (see inset). These fast oscillations have a characteristic frequency of $52.7 \mathrm{GHz}$ (Fig. $4.10 \mathrm{~b}$ ) and can be interpreted as a stress wave propagating perpendicularly to the sample plane, through the $\mathrm{CoFeB}$ thin film. After the excitation, the incoherent reflectivity dynamics decays exponentially to its initial value. Moreover, the elastic dynamics is not influenced by the milling of $a(a=3.5 \mu \mathrm{m}, d=1 \mu \mathrm{m})$ magnonic crystal on such film ${ }^{77}$.

When reducing the lattice constant and the antidot diameter by a factor of two $(\mathrm{a}=1.75 \mu \mathrm{m}, \mathrm{d}=0.5 \mu \mathrm{m})$, so that the filling fraction remains constant $(\approx 6 \%)$, the elastic dynamics is strongly changed. As shown in Fig. 4.11a. the most notorious feature is that the decay of reflectivity dynamics is much slower than that in the continuous film. Additionally to the fast $56.9 \mathrm{GHz}$ oscillations (analogous to those on continuous film, as shown in the inset), three broad, low-frequency modes $\left(\omega_{1.75 \mu \mathrm{m}, 1}=1.4 \mathrm{GHz}, \omega_{1.75 \mu \mathrm{m}, 2}=3.1 \mathrm{GHz}\right.$ and $\omega_{1.75 \mu \mathrm{m}, 4}=7.8 \mathrm{GHz}$ ) are detected in the Fourier-transformed signal (see Fig. $4.11 \mathrm{~b}$. Moreover, Fig 4.12a shows the reflectivity dynamics on a $(\mathrm{a}=700 \mathrm{~nm}$ , $\mathrm{d}=200 \mathrm{~nm}$ ) magnonic crystal, in which the filling fraction was maintained the same as above, although the antidot diameter and the distance between antidots were decreased by a factor of 2.5. We can observe that, in analogy to the $\mathrm{a}=1.75 \mu \mathrm{m}$ antidot lattice, slow elastic dynamics are detected in the reflectivity signal, in addition to fast oscillations. These fast oscillations are now shifted to slightly higher frequencies in the Fourier spectrum. Since the three antidot lattices were milled on near locations on the same sample film, frequency variations due to a thickness gradient can be excluded.

On the other hand, the slow oscillations frequencies on $a=700 \mathrm{~nm}$ antidot lattice are quite different from those found on $a=1.75 \mu \mathrm{m}$ lattice. In general, the frequencies are shifted in this case to higher values, namely $\omega_{700 \mathrm{~nm}, 1}=$ $1.17 \mathrm{GHz}, \omega_{700 \mathrm{~nm}, 2}=7.1 \mathrm{GHz}, \omega_{700 \mathrm{~nm}, 3}=10.3 \mathrm{GH} z$ and $\omega_{700 \mathrm{~nm}, 4}=17.22 \mathrm{GHz}$, as shown in Fig $4.12 \mathrm{~b}$. 


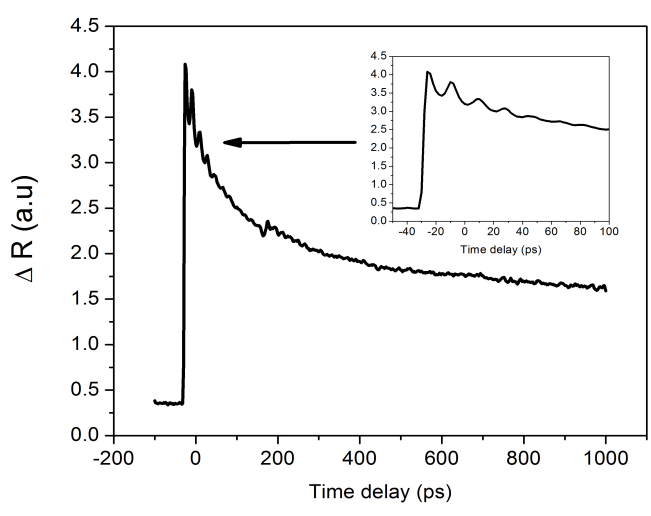

(a)

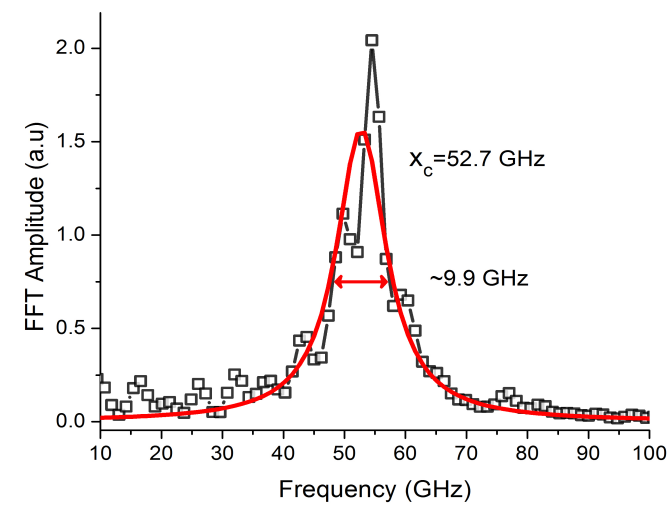

(b)

Figure 4.10: a) Reflectivity dynamics on the continuous 50-nm CoFeB film. Fast oscillations can be observed in the first 100 ps (inset). b) Fast Fourier transform of the signal after the subtraction of the incoherent (exponential) background. A wide peak can be seen around $52.7 \mathrm{GHz}$. Red line is a Lorentzian fit to the data.

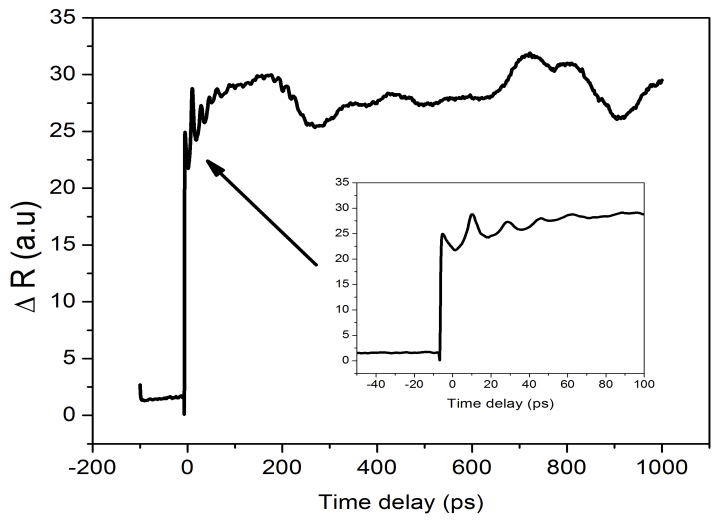

(a)

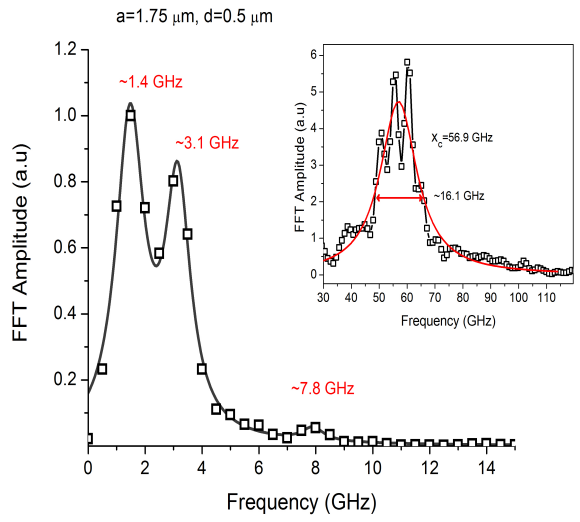

(b)

Figure 4.11: a) Reflectivity dynamics on a magnonic crystal $(a=1.75 \mu \mathrm{m}, \mathrm{d}=$ $0.5 \mu \mathrm{m}$ ) in $50-\mathrm{nm}$ CoFeB film. Fast oscillations can be observed in the first $100 \mathrm{ps}$ (inset). In contrast to dynamics on continuous film (Fig. $4.10 \mathrm{a}$ ) slow oscillations with a large amplitude can be observed for up to 1 ns. b) Fast Fourier transform of the signal after the subtraction of the incoherent (exponential) background. In addition to a peak at $56.9 \mathrm{GHz}$ (inset), low-frequency modes $(1.4 \mathrm{GHz}, 3.1$ $\mathrm{GHz}$ and $7.8 \mathrm{GHz}$ ) are detected. Solid lines are Lorentzian fits to the data. 


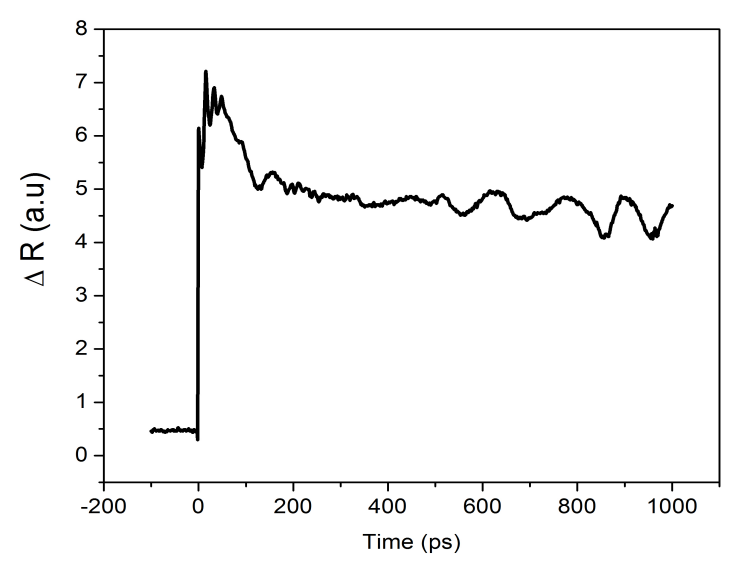

(a)

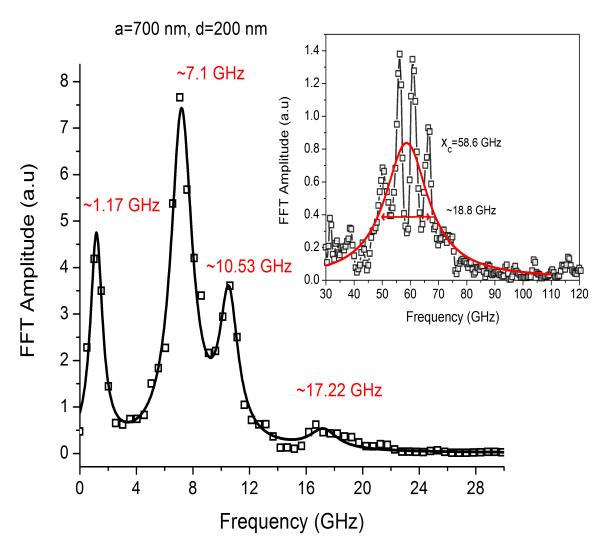

(b)

Figure 4.12: a) Reflectivity dynamics on a magnonic crystal ( $\mathrm{a}=700 \mathrm{~nm}$, $\mathrm{d}=200 \mathrm{~nm}$ ) in a 50-nm CoFeB film. In analogy to Fig 4.11a large-amplitude, slow oscillations can be observed for up to $1 \mathrm{~ns}$. b) Fast-Fourier transform of the signal after the subtraction of the incoherent (exponential) background. In addition to a peak at $58.6 \mathrm{GHz}$ (inset), low-frequency modes $(1.17 \mathrm{GHz}, 7.1 \mathrm{GHz}$, 10.3 GHz and 17.22 GHz) are detected. Solid lines are Lorentzian fits to the data.

Moreover, a correspondence between the frequency peaks can be observed, in this case:

$$
\begin{gathered}
\frac{\omega_{700 \mathrm{~nm}, 4}}{\omega_{1.75 \mu \mathrm{m}, 4}}=\frac{17.22 \mathrm{GHz}}{7.8 \mathrm{GHz}}=2.20, \\
\frac{\omega_{700 \mathrm{~nm}, 2}}{\omega_{1.75 \mu \mathrm{m}, 2}}=\frac{7.1 \mathrm{GHz}}{3.1 \mathrm{GHz}}=2.29 .
\end{gathered}
$$

This means that some peaks were shifted by a factor of $\approx 2.2$ towards higher frequencies, when the antidot lattice constant was decreased by a factor of

$$
\frac{1.75 \mu \mathrm{m}}{700 \mathrm{~nm}}=2.5
$$

This way, we should expect a peak at $\sim 4.68 \mathrm{GHz}$ in the reflectivity Fourier spectrum of $a=1.75 \mu \mathrm{m}$ lattice. However, as we can see in Fig. 4.11b, such a peak would be overlapped with the high amplitude $3.1 \mathrm{GHz}$ frequency. Moreover, the two lowest frequencies in both reflectivity spectra could not be resolved, since our resolution is limited to $1 \mathrm{GHz}$ (1 ns delay stage).

The proportionality factor between the frequency peaks, that approximately corresponds to the decrease in antidot lattice constant, strongly suggests that surface elastic modes were excited on the antidot lattice. However it must be 
noted that the antidot size was also decreased by the same factor. The antidot can also act as an emitter of elastic waves when the film is excited by the laser pump. This way, it is not clear whether the elastic waves are excited in the CoFeB film or the silicon substrate (or both) and which wave vector should be associated with them (the lattice constant or the antidot size). This makes the determination of the elastic wave velocity a difficult task, that lies beyond the focus of this work.

\subsubsection{Magnetization dynamics}

The main focus of this section is to study the magnetization dynamics on submicron magnonic crystals. As we will see, not only the elastic dynamics are influenced by reducing the lattice constant, but also the magnetization dynamics, due to changes in the effective field landscape.

Magnetization dynamics frequencies, as function of applied magnetic field on $a=1.75 \mu \mathrm{m}$ and $\mathrm{a}=700 \mathrm{~nm}$ magnonic crystals, were determined for different orientations of external magnetic field with respect to the lattice vector. As shown in Fig. 4.13, when the external field is applied along the lattice vector, neither of the magnonic crystals show a clear DE mode, but PSSW and Kittel modes are present. In the case of $a=1.75 \mu \mathrm{m}$ the expected DE frequencies lie very close to the PSSW frequencies as expected (see Fig 4.6 in section 4.2 and the discussion therein). So, the observed mode should be at least broader than the PSSW mode. On the other hand, on $a=700 \mathrm{~nm}$ antidot lattice, a highfrequency DE mode, above PSSW mode frequency would be expected, but is not observed.

Moreover, the Kittel and PSSW modes are practically lost for $\mathrm{a}=700 \mathrm{~nm}$ antidot lattice at low external magnetic fields $\left(\mu_{0} \mathrm{H}_{x} \leqslant 60 \mathrm{mT}\right)$.

The most feasible explanation for the absence of DE mode on the sub-micron antidot lattice is the modification of the effective internal field due to an increasing antidot proximity and the resulting overlapping dipolar fields of each antidot. As shown in Fig. 4.14d, the internal field in a $a=3.5 \mu \mathrm{m}$ lattice with an external field applied perpendicular to the lattice vector presents large stripes of nearly constant effective field, where delocalized spin-wave modes in DEgeometry can be excited. This is in contrast to Fig. 4.14c, where internal magnetic field in the sample is strongly decreased with respect to the applied magnetic field. This is manifest as more bluish (reddish) areas, that indicate areas of decreased (increased) effective field with respect to the applied $130 \mathrm{mT}$ field in the $x$-direction.

As shown in 4.15, the effective magnetic field between antidots in a lattice with $a=1.75 \mu \mathrm{m}$ is further reduced, as compared to $a=3.5 \mu \mathrm{m}$, by approxi- 


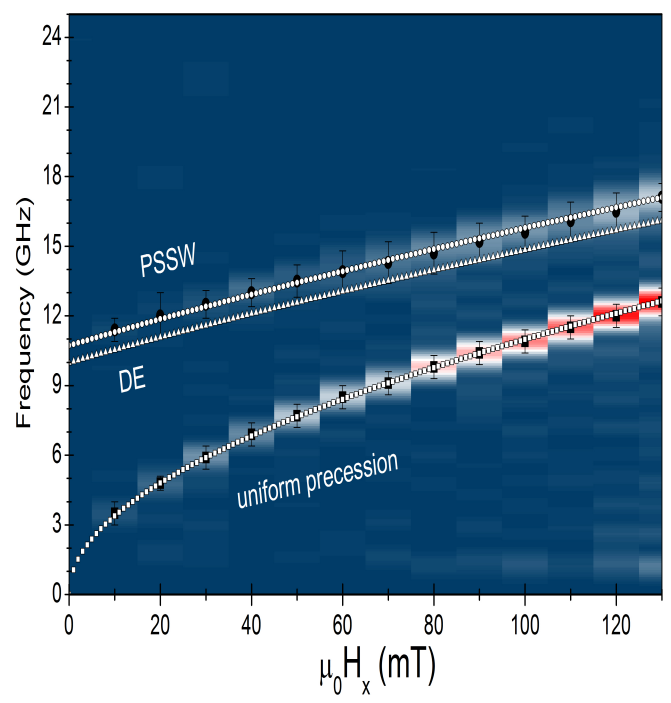

(a)

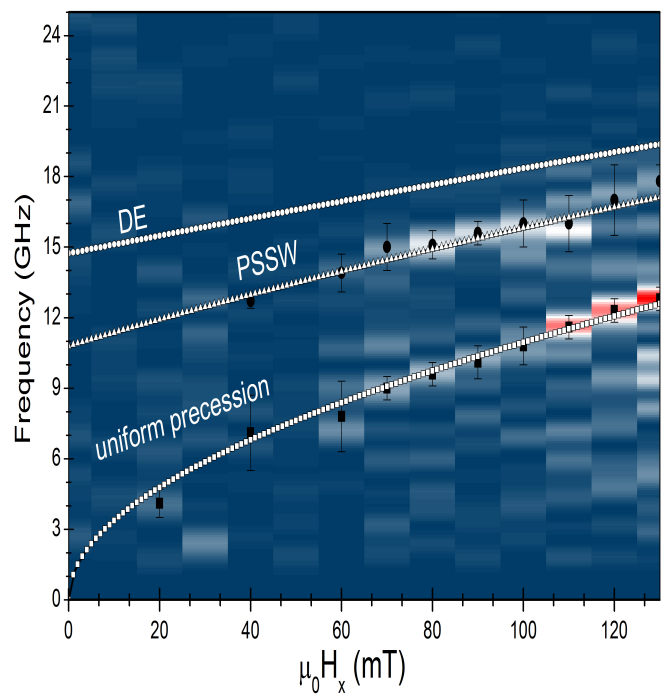

(b)

Figure 4.13: FFT correlated amplitude (in color code) of magnetization dynamics in (a) $a=1.75 \mu \mathrm{m}, d=0.5 \mu \mathrm{m}$ and (b) $\mathrm{a}=700 \mathrm{~nm}, \mathrm{~d}=200 \mathrm{~nm}$ magnonic crystals, with an external magnetic field applied along the lattice constant. Two modes are identified in both cases: Kittel (squares) and PSSW (circles) modes. Dotted lines represent the expected Kittel, DE and PSSW mode frequencies.

mately $2 \%$. Moreover, it becomes more inhomogeneous (as can be observed in the changing curvature). If we suppose that the internal field is homogeneous when it does not deviate from its maximum value by more than $5 \%$ (see dotted lines in Fig. 4.15), then we can calculate that the effective width of homogeneous internal field area is reduced by approximately $0.5 \mu \mathrm{m}$ or $28.5 \%$ of the unit cell, although the filling fraction was maintained constant.

This way, decreasing the extension of areas with homogeneous effective field hinders the excitation of delocalized spin-wave modes. Such effect would be even more pronounced in $a=700 \mathrm{~nm}$ (not shown) magnonic crystal, since the antidots are even closer to each other, creating a even larger overlap between their dipolar fields.

When comparing the internal fields in different antidot lattices at $45^{\circ}$ (to the lattice vector) applied magnetic field, the lack of homogeneity in the direction of $\mathrm{DE}$ wave vector becomes even more evident (see figs. 4.14a and 4.14b). In this case, regions of decreased and enhanced effective fields alternate with each other along the direction of DE wave vector. Excitation of the DE mode in this area would require frequency up-conversion in the regions with enhanced magnetic field. This allows us to conclude that DE mode should not be expected 


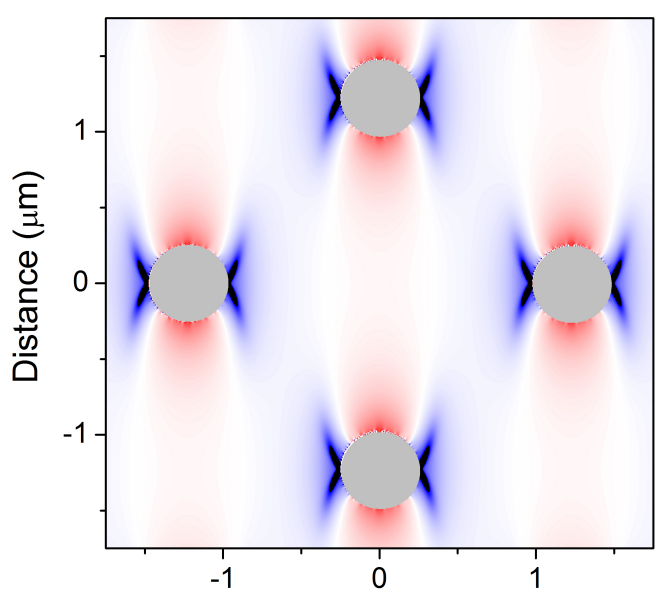

(a)

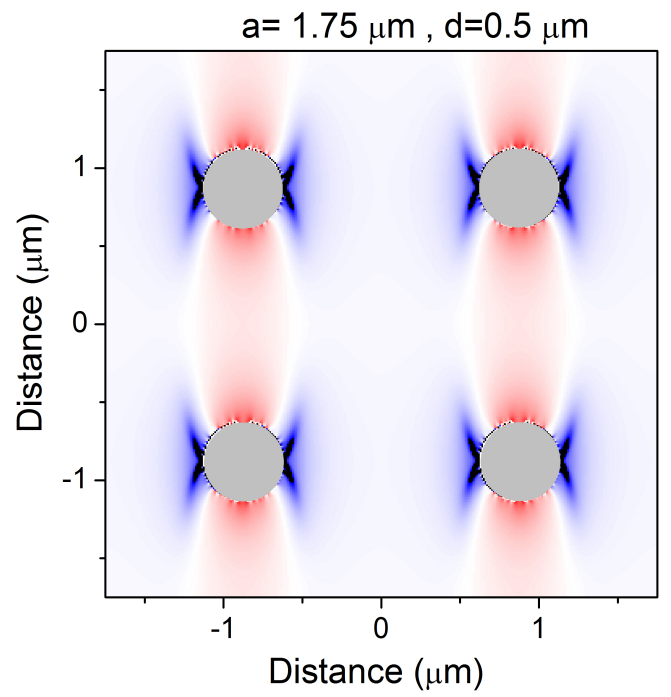

(c)

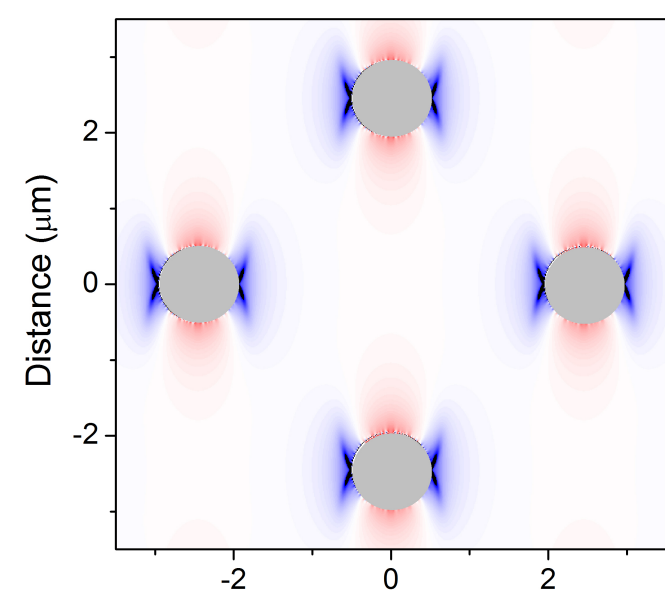

(b)

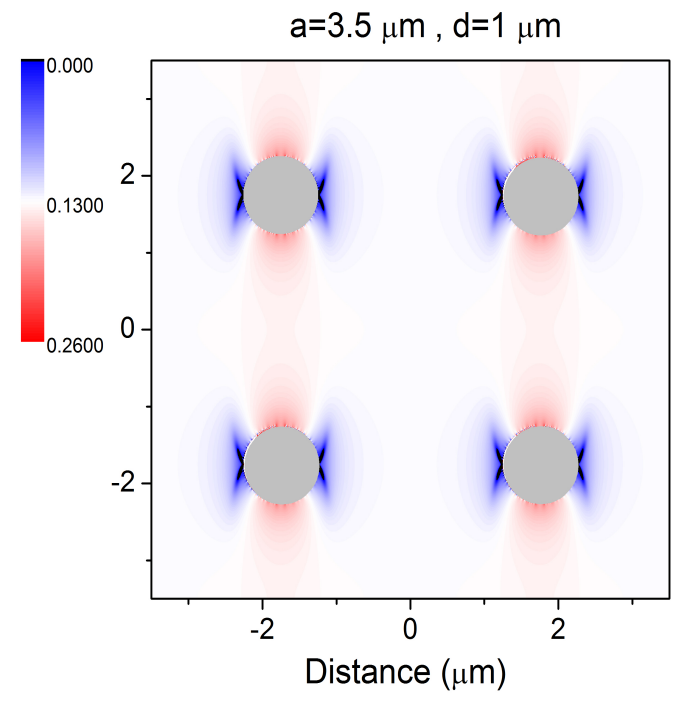

(d)

Figure 4.14: Effective field (in color code) calculated by mumax package for $\mathrm{a}, \mathrm{c}) \mathrm{a}=1.75 \mu \mathrm{m}, \mathrm{d}=0.5 \mu \mathrm{m}$ and $\mathrm{b}, \mathrm{d}) \mathrm{a}=3.5 \mu \mathrm{m}, \mathrm{d}=1.75 \mu \mathrm{m}$ magnonic crystal. An external $150 \mathrm{mT}$ field is applied at $30^{\circ}$ to the sample plane and at $90^{\circ}(\mathrm{c}, \mathrm{d})$ and $45^{\circ}(\mathrm{a}, \mathrm{b})$ to magnonic crystal lattice vector. Gray circles represent the antidot.

either when external field is applied at $45^{\circ}$ or along the lattice vector in submicron antidot lattices. However, as we can see in figs. 4.16 and 4.17, not only the DE mode is not detected, but both antidot lattices present a very peculiar magnetization dynamics (if at all).

As shown in Fig. 4.16 many low-frequency modes are detected in the FFT 


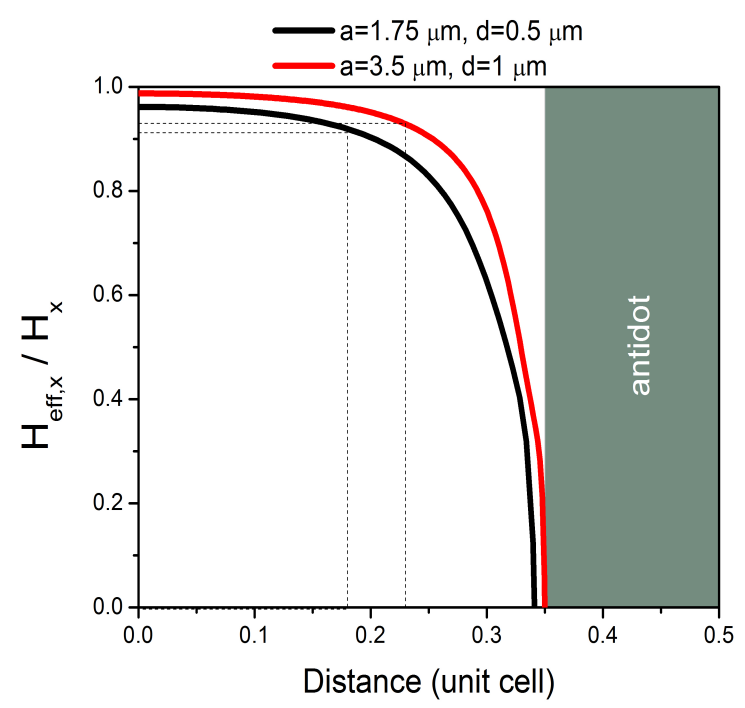

Figure 4.15: Effective field amplitude as calculated in figs. $4.14 \mathrm{c}$ and $4.14 \mathrm{~d}$ along the $x$-direction between the antidots and normalized to the corresponding unit cell length. Green area indicates the antidot. Dotted lines indicate the area where the internal magnetic field variation is less than $5 \%$ of its maximum value.

spectrum of the magnetization dynamics on $a=1.75 \mu \mathrm{m}$ magnonic crystal. These low-frequency modes show no clear dependence on the applied magnetic field, and do not match any of the expected frequencies for this film. Notably, they appear to be bounded by the Kittel mode at each applied field.

Moreover, their amplitude is quite large. Only at $\mu_{0} \mathrm{H}_{x} \geqslant 80 \mathrm{mT}$ a mode matching PSSW frequency and a mode whose frequency lies in between the expected Kittel and DE-mode frequencies seem to emerge with an amplitude comparable to the low-frequency modes.

It must be noted that both Kittel and PSSW modes seem to appear only after a frequency threshold above $9 \mathrm{GHz}$. As can be seen in the inset of Fig. 4.16, this threshold also corresponds to the upper limit to the elastic modes frequencies detected on the same structure. This hints that elastic dynamics induced by the antidot lattice could strongly interfere with magnetization oscillations.

We have previously seen that the characteristic frequencies of elastic dynamics shift towards higher values when the antidot lattice constant is reduced to $\mathrm{a}=700 \mathrm{~nm}$. The magnetization dynamics in this case (see Fig. 4.17) shows a mode at $\sim 9 \mathrm{GHz}$, which frequency is practically constant up to $100 \mathrm{mT}$, where modes with frequencies nearly matching the expected Kittel and PSSW modes start to appear. 


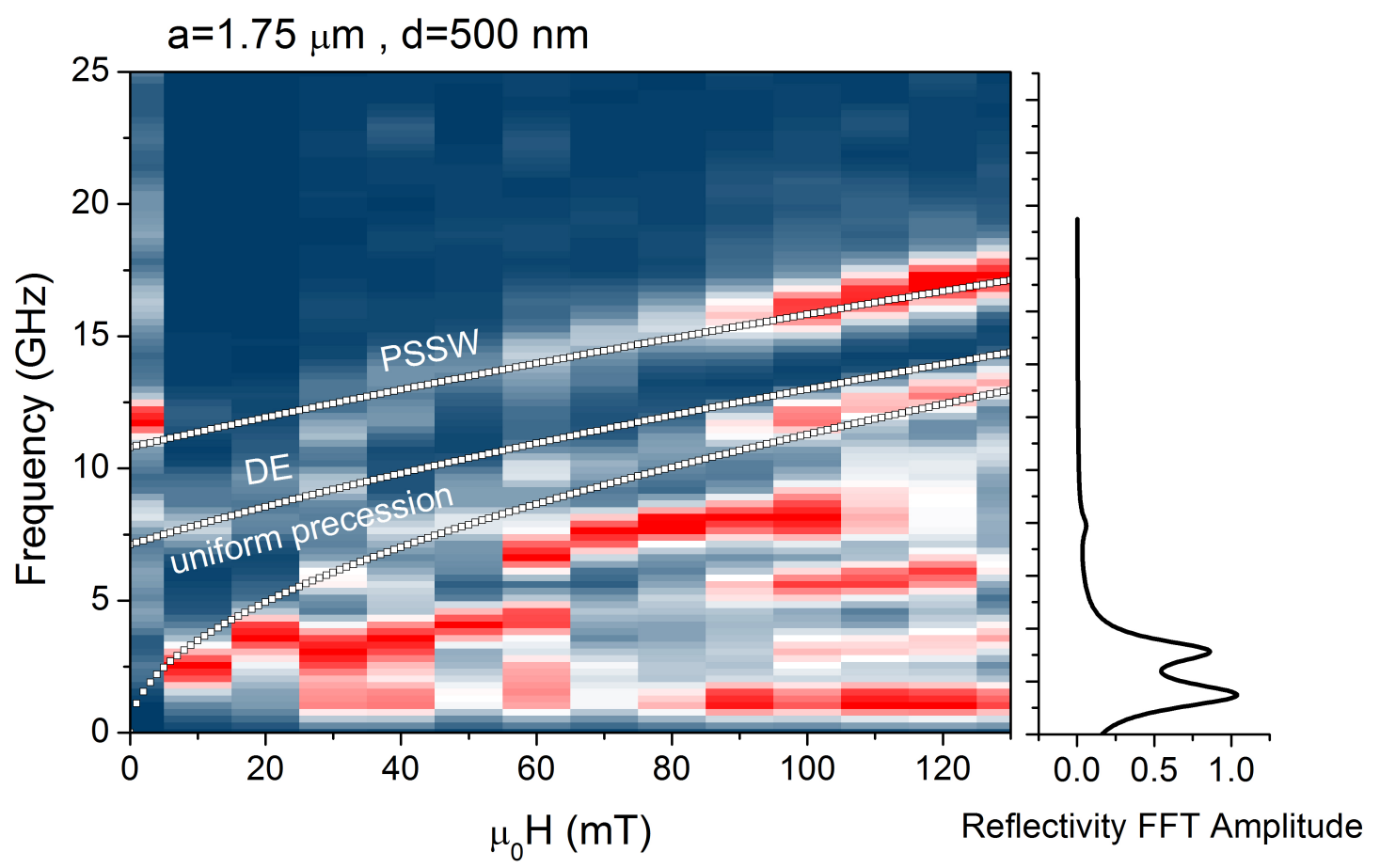

Figure 4.16: FFT correlated amplitude (in color code) of magnetization dynamics in $\mathrm{a}=1.75 \mu \mathrm{m}, \mathrm{d}=0.5 \mu \mathrm{m}$ magnonic crystal, with an external magnetic field applied at $45^{\circ}$ to the lattice constant. Dotted lines represent the expected frequencies of Kittel, DE and PSSW modes. Inset on the right shows the FFT amplitude of the frequencies found in reflectivity signal.

\subsubsection{Discussion}

Here the main results of this section are discussed and compared to previously reported magnonic spin wave modes in Ref. 11 in antidot lattices of similar CoFeB composition and thickness, although with a larger filling fraction (12\%) i.e., a larger antidot diameter than in our magnonic crystals. The characteristic width and thickness of spin-wave channels, as well as their aspect ratios are summarized in the table 4.1 .

One of the most relevant differences with Ref. 11 is that, in these antidot lattices, no evidence of surface acoustic waves in the reflectivity signal was found, although a weak $0.5 \mathrm{GHz}$ oscillation is mentioned. This points to the antidot diameter, rather than the distance between antidots, as the main parameter that regulates the excitation of surface acoustic waves. This is in agreement with the surface acoustic waves generated in an array of nickel dots in Ref. 84 .

We start by comparing the magnetization dynamics on lattices magnetized along the lattice vector and at $45^{\circ}$ to it. We can note that the Kittel and PSSW 


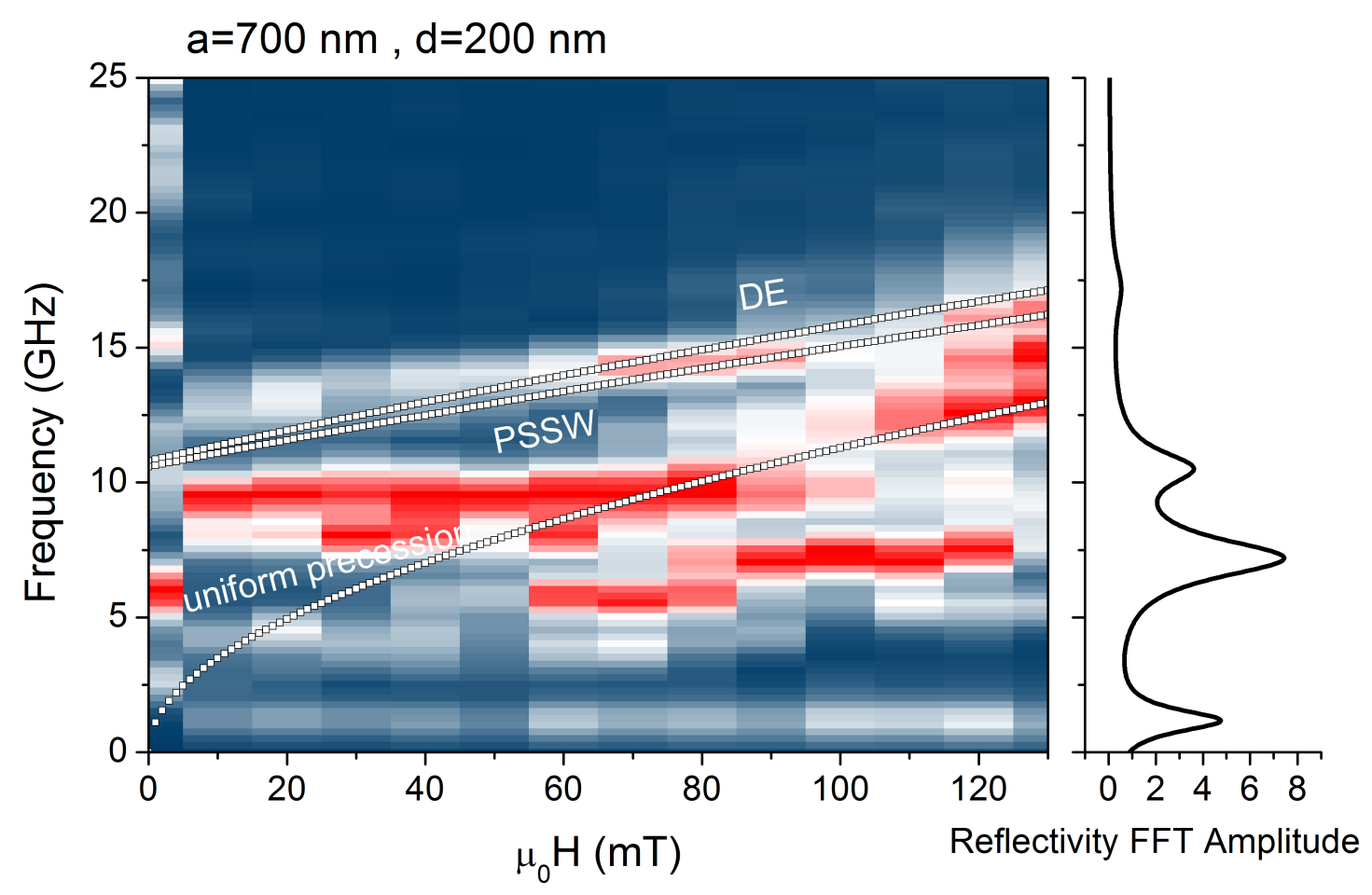

Figure 4.17: FFT correlated amplitude (in color code) of magnetization dynamics in $\mathrm{a}=700 \mathrm{~nm}, \mathrm{~d}=200 \mathrm{~nm}$ magnonic crystal, with an external magnetic field applied at $45^{\circ}$ to the lattice constant. Dotted lines represent the expected frequencies of Kittel, DE and PSSW modes. Inset on the right shows the FFT amplitude of the frequencies found in reflectivity signal.

\begin{tabular}{lccr}
\hline \hline$(\mathrm{a}, \mathrm{d})$ & $\mathrm{t}(\mathrm{nm})$ & $\mathrm{w}(\mu \mathrm{m})$ & $\frac{\mathrm{t}}{w}$ \\
\hline $3.5 \mu \mathrm{m}, 1 \mu \mathrm{m}$ & 50 & 2.5 & 0.02 \\
$3.5 \mu \mathrm{m}, 1 \mu \mathrm{m}$ & 82.5 & 2.5 & 0.033 \\
$1.75 \mu \mathrm{m}, 0.5 \mu \mathrm{m}$ & 50 & 1.25 & 0.04 \\
$700 \mathrm{~nm}, 200 \mathrm{~nm}$ & 50 & 0.5 & 0.1 \\
\hline \hline
\end{tabular}

Table 4.1: Thickness $(\mathrm{t})$, width $(\mathrm{w})$, aspect ratios $(\mathrm{t} / \mathrm{w})$ of the magnonic crystals with lattice constant $a$ and antidot diameter $d$ reported in this work

in Fig. 4.13a are present even at very low applied fields, while in $a=700 \mathrm{~nm}$ magnonic crystal the onset occurs at $\sim 60 \mathrm{mT}$. In contrast, when the field is applied at $45^{\circ}$, the Kittel and PSSW modes are detected at $\mathrm{H}>100 \mathrm{mT}$ in $a=1.75 \mu \mathrm{m}$, and not detected at all in $a=700 \mathrm{~nm}$ magnonic crystal. This points to a larger spatial inhomogeneity of the internal field when the mag- 
netic field is applied along a high-symmetry direction. This can be also seen by comparing Fig. $4.14 \mathrm{a}$ and $4.14 \mathrm{c}$. As reported in Ref. 37, antidot lattices with large aspect ratio should present localized modes which frequency lies below the Kittel mode, further decreasing with increasing aspect ratio. However, in this configuration we find no evidence of excitation of such modes even at low field and high aspect ratios, in contrast to the $45^{\circ}$ configuration.

The low-frequency modes detected in $a=1.75 \mu \mathrm{m}$ with external field at $45^{\circ}$ to the lattice vector, that show a weak dependence on external field, similarly to those reported in Ref. 11, can be attributed to modes localized in areas of inhomogeneous internal field. However, an important difference is that the detected modes show a weak dependence on the external field and are contained below the Kittel mode. They also show a step-like increase in frequency at $60 \mathrm{mT}$ and $100 \mathrm{mT}$, where they finally reach and follow the Kittel mode.

On the other hand, as the aspect ratio is increased to 0.1 in $a=700 \mathrm{~nm}$ lattice, a single mode emerges above the Kittel frequency at low fields with nearly constant frequency up to $100 \mathrm{mT}$. Moreover, its frequency stays practically constant, suggesting that it could be localized in areas of large internal field in between the neighboring antidots.

Another possible explanation of the results in this section is an interplay between the elastic and magnetization oscillation modes. The surface elastic waves would alter the effective magnetic field in the sample in a time-dependent manner, thus contributing with an additional strain-induced anisotropy field. Moreover, an elastic wave couples most efficiently to the magnetization lying at $45^{\circ} \sqrt{85}$ to its wave vector. This would be a possible explanation for such different magnetization dynamics when changing the direction of applied magnetic field.

\subsection{Heating effects on properties of magnetization oscillation}

In this section we address the generation of a spin-wave trap on a magnonic crystal, using a laser-induced temperature gradient. Most of the results reported in this section can also be found in Ref. 65.

The use of laser pulses makes unnecessary any direct contact with the sample, in contrast to, for example, a Peltier element used in Ref. 86. Pulsed laser power can be increased so that not only magnetization dynamics is excited, but also a temperature profile is created with the same pulse. This temperature profile will act as a trap for the spin-waves excited on the magnonic crystal, locally modifying their dispersion characteristics. 
In order to determine the pulse power needed to generate a significant heat gradient, the numerical simulation package COMSOL was used to calculate the temperature profile after laser excitation in a $50 \mathrm{~nm} \mathrm{CoFeB}$ thin film on a thick $\mathrm{Si}(100)$ substrate that acts as a heat sink. Energy is deposited by a $50 \mathrm{fs}$ laser pulse with a Gaussian profile in the sample plane and a $60 \mu \mathrm{m}$ FWHM. The energy per pulse amounts to $1.6 \mu \mathrm{J}$ with a total fluence of $13 \mathrm{~mJ} / \mathrm{cm}^{2}$. These parameters correspond to the experimental values we used. Further details on COMSOL simulations can be found in Ref. 65.

Once the electrons are thermalized after optical excitation $(\approx 100 \mathrm{fs})$, and the phonon and spin subsystems are equilibrated $(\approx 1 \mathrm{ps})$, we can consider the effective temperature of the magnetic system. As can be seen in Fig. 4.18 (right), the laser pulse produces a rapid rise in temperature in the first $100 \mathrm{ps}$, so that during this time spatial and temporal heat gradients are quite large. Soon after, thermal equilibrium is reached and temperature remains considerably above $400 \mathrm{~K}$ at the center of pump spot for more than $1 \mathrm{~ns}$. Since the sample thickness (50 nm) is much smaller than the pump spot FWHM, the temperature profile has a negligible depth variation, but it changes significantly in the sample plane, following the Gaussian shape of the pump spot (Fig. 4.18 left). Moreover, the FWHM of the Gaussian temperature profile does not change over time, i.e., no significant heat transport takes place at micrometer scale. This way, the COMSOL simulations allow us to conclude that a temperature profile can be effectively created by short laser pulses over a large (tens of micrometers) area, that persist for up to $1 \mathrm{~ns}$, comparable with spin-wave lifetimes.
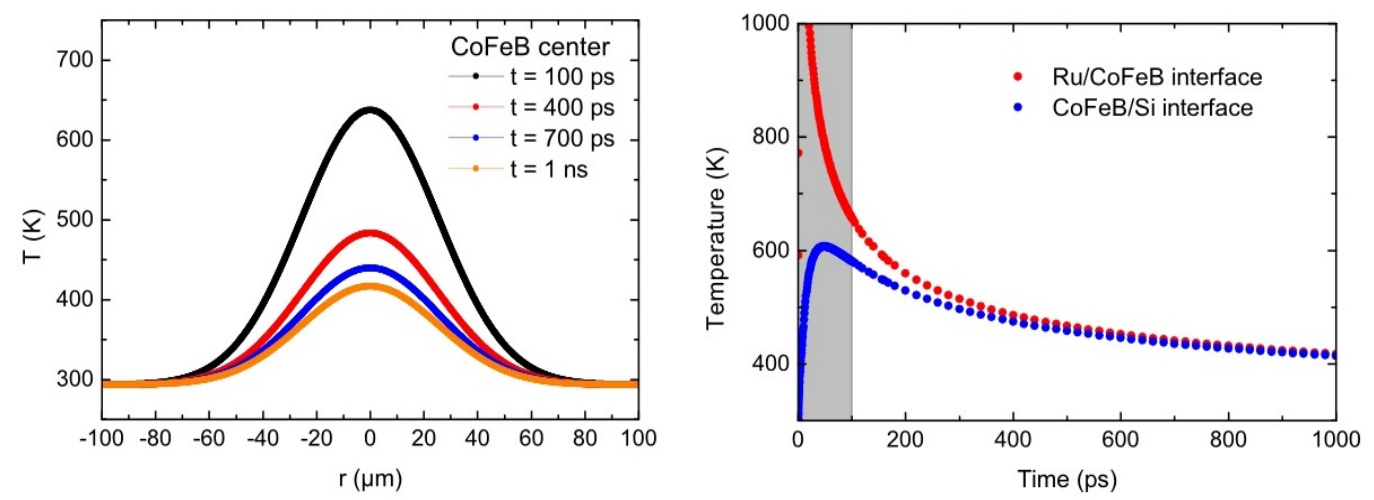

Figure 4.18: Simulated temperature distribution (left) on the sample plane at different times in the film midsection and (right) time evolution of the temperature at the top $(\mathrm{Ru} / \mathrm{CoFeB})$ and bottom $(\mathrm{CoFeB} / \mathrm{Si})$ interface. Gray-shadowed area indicates the first 100 ps, when the heat gradient is large.

According to the COMSOL simulations annealing temperatures are reached $\left(400^{\circ} \mathrm{C}\right)$, at which $\mathrm{CoFeB}$ might crystallize and change its structural and mag- 
netic properties during the experiment 87 . However, considering the low repetition rate of the laser $(250 \mathrm{kHz})$, there are at least $4 \mu \mathrm{s}$ between the ultrafast laser pulses, much longer than the time the sample remains heated (in the order of tens of nanoseconds). This way, the sample is cooled down between pulses and remains at relatively low temperatures most of the time. Moreover, any structural changes due to reordering or crystallization should be evident in the hysteresis curves and coercive field values. However, as shown in Fig. 3.3b. the hysteresis measured before and after the experiment show no significant changes. This allows us to conclude that high temperatures can be reached by ultrafast laser heating, albeit for a very short time, so that no annealing effects can take place.

Spatially-resolved measurement of magnetization dynamics were performed along the laser excitation spot in a $50-\mathrm{nm}$ CoFeB thin film, at $130 \mathrm{mT}$ external magnetic field applied at $20^{\circ}$ to the film plane. The pump and probe FWHM amount to $93 \mu \mathrm{m}$ and $24 \mu \mathrm{m}$. Moreover, two relative scanning directions are considered: parallel and perpendicular to the in-plane projection of the applied field.

Fourier-transformed magnetization dynamics data is presented in Fig 4.19 (left). As can be observed, two modes are identified as Kittel and PSSW modes, whose frequency increases towards the edges of the excitation spot. The Kittel mode is shifted by $\sim 0.6 \mathrm{GHz}(4.5 \%)$ with respect to the frequency on the spot edge, while PSSW mode has a larger, $1.1 \mathrm{GHz}(5.6 \%)$ frequency shift.

The Kittel mode frequency follows the Gaussian profile in both parallel and perpendicular (to the applied field) scanning directions, which FWHM is comparable to that of the excitation spot and the minimum frequency corresponds to the center with maximum pump intensity (temperature). Slight deviation in the FWHM values might be due to an ellipticity of the pump spot.

From Eq. 2.8 we know that Kittel mode frequency depends on the local saturation magnetization, which in turn, is temperature dependent according to the Curie-Weiss law. The variation of mode frequency $\Delta \omega$ with respect to small changes in saturation magnetization $\Delta \mu_{0} M$ can be calculated by deriving Eq. 2.11 with respect to $\mu_{0} M$ :

$$
\begin{aligned}
& \frac{\Delta \omega_{\text {PSSW }}}{\omega_{\text {PSSW }}}=\frac{\Delta \mu_{0} M}{2 \mu_{0} M}\left[\frac{\omega_{M}}{\omega_{H}+\omega_{A}+\omega_{M}}-\omega_{A}\left(\frac{1}{\omega_{H}+\omega_{A}}+\frac{1}{\omega_{H}+\omega_{A}+\omega_{M}}\right)\right] \\
& \frac{\Delta \omega_{\text {Kittel }}}{\omega_{\text {Kittel }}}=\frac{\Delta \mu_{0} M}{2 \mu_{0} M} \frac{\omega_{M}}{\omega_{M}+\omega_{H}}
\end{aligned}
$$

We can note that the expression for the variation of Kittel mode frequency (Eq. 4.6) is recovered by setting $n=0, \omega_{A}=0$ in the expression for the PSSW 

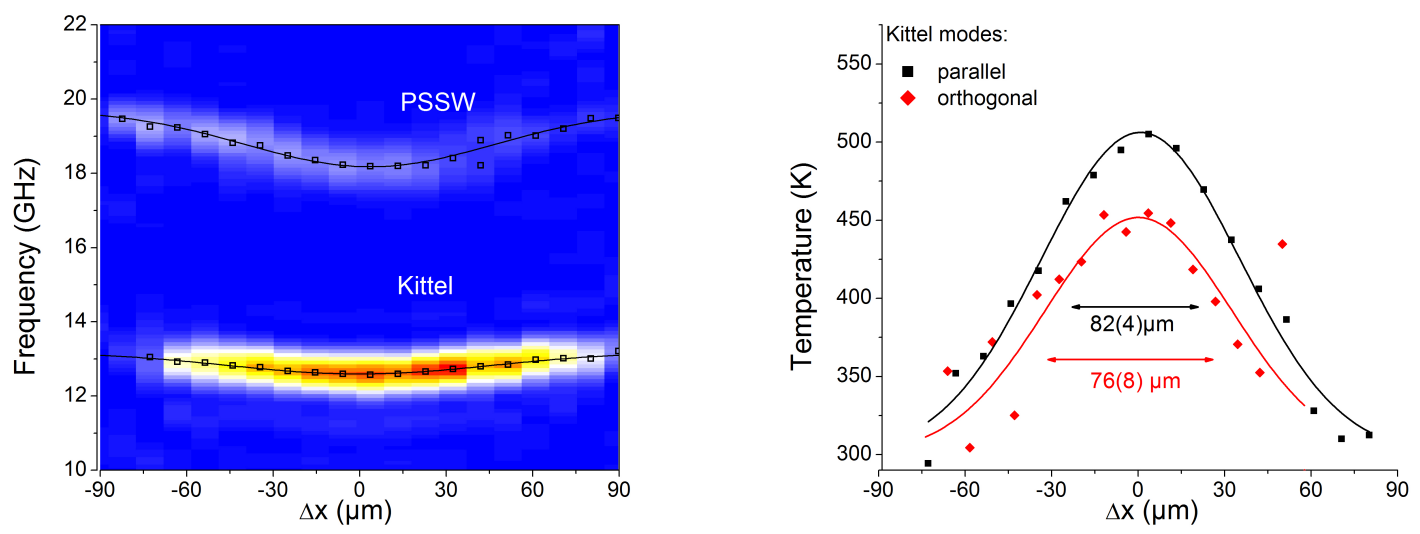

Figure 4.19: Magnetization dynamics on a continuous film across the pump spot (left). Open squares represent fitted peak frequencies. The temperature of the spin system is derived (right). Diamonds correspond to the probe scanning along the pump spot in a direction orthogonal to the applied field, squares depict a parallel displacement. Solid lines are Gaussian fits to the data. Curves are offset so that the frequency dip of the Kittel mode is centered at $\Delta x=0$.

frequency (Eq. 4.5). In general, $\Delta \omega_{\mathrm{PSSW}}$ should be smaller than the corresponding $\Delta \omega_{\text {Kittel }}$ due to a negative contribution term proportional to $\omega_{A}$ i.e., the exchange field frequency proportional to exchange constant A. However, the experimental data shows that the PSSW frequency shift is larger than the one expected due to variations in $M_{s}$ only, as result of changing the temperature. This suggests a more complicated temperature dependence of the PSSW mode, that includes a temperature dependence of the exchange constant A.

Using VSM measurements reported in Fig. 3.3a, local temperature is calculated (as shown in Fig. 4.19 right) taking into account the Kittel frequency shift data. As we can see, the calculated temperature profile agrees reasonably well with the COMSOL simulation (Fig. 4.18), yielding a similar FWHM.

On a magnonic crystal, as was shown in previous sections, an additional DE mode is expected in the magnetization dynamics spectra. In contrast to PSSW and Kittel modes that have no in-plane wave vector component, DE wave vector lies either perpendicular or at $45^{\circ}$ to the magnetization and its magnitude is inversely proportional to the lattice constant of magnonic crystal $k=\pi / a$. The most significant density of states is expected for DE mode close to Brillouin zone boundary, where the bands are flattening ${ }^{1130}$ leading to a decreased DE group velocity. However, the excitation mechanism is broadband (as discussed in section 2.3.2), and within a $0.5 \mathrm{GHz}$ bandwidth propagation lengths more than $1.3 \mu \mathrm{m}$ are expected in the $1 \mathrm{~ns}$ observation time. This way, by comparing 
the FWHM of the localized Kittel and extended DE modes amplitudes along the excitation spot, surface propagation characteristics can be determined.
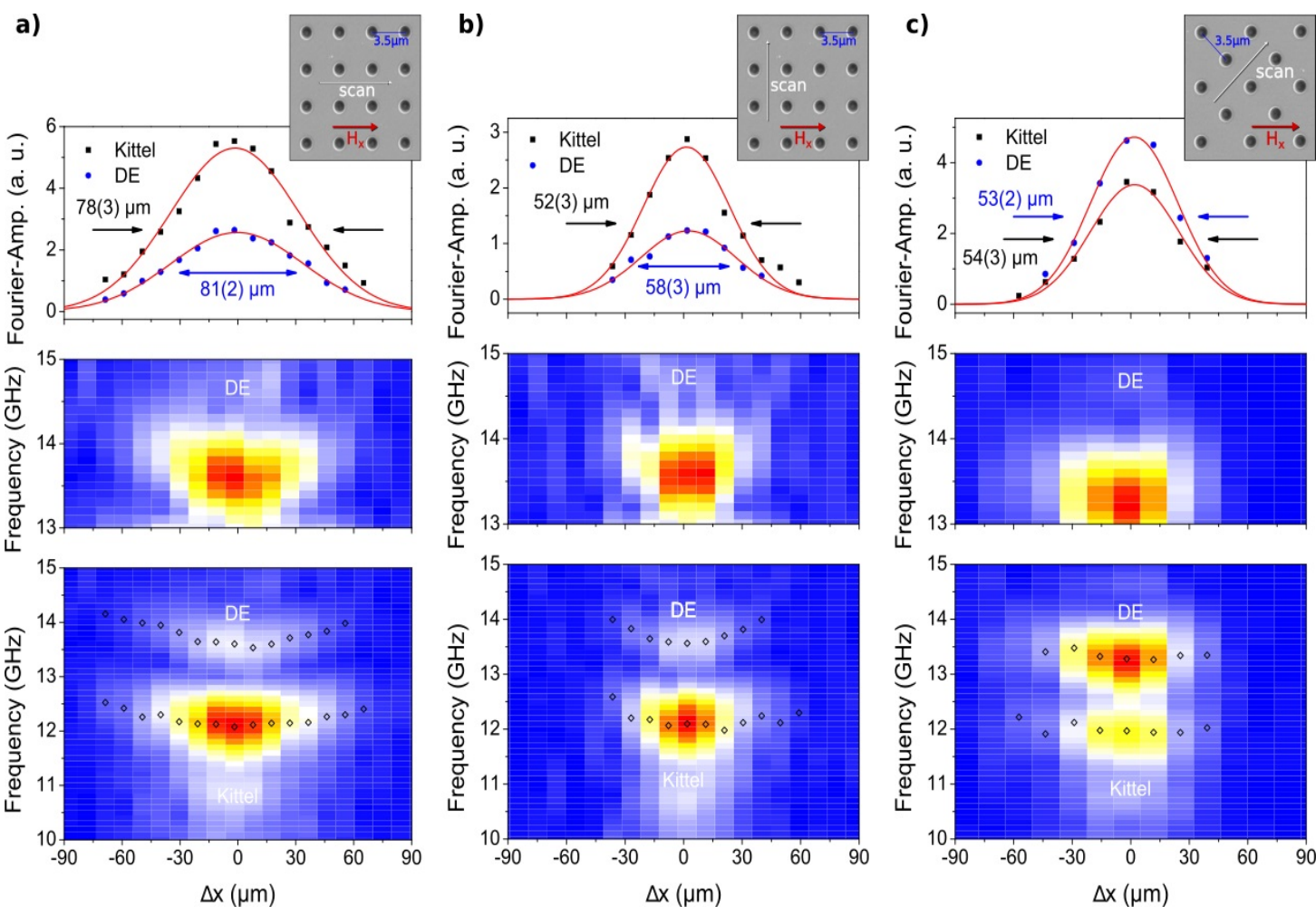

Figure 4.20: Magnetization dynamics on a magnonic crystal. Bottom: Fourierspectrum in analogy to Fig. 4.19 (top) with the additional magnonic DamonEshbach (DE) mode. Above, the DE-mode is shown with an adapted color code for better visibility. The Fourier amplitude for fundamental Kittel mode (squares) and dipolar DE mode (circles) is plotted along the excitation spot. Top: Scanning direction relative to the applied field and to the magnonic crystal.

Magnetization dynamics measurements on the $(a=3.5 \mu \mathrm{m}, \mathrm{d}=1 \mu \mathrm{m})$ magnonic crystal saturated by an $130 \mathrm{mT}$ external magnetic field tilted $20^{\circ}$ with respect to the surface are shown in Fig. 4.20. Due to a rich magnetization dynamics in this structure, different scanning directions can be chosen: a) parallel, b) orthogonal and c) at $45^{\circ}$ to the applied field, as shown in the upper panel.

The additional magnonic DE-mode is visible (bottom and middle panel in Fig. 4.20) along the pump profile. Analogously to Kittel and PSSW modes, the DE mode amplitude shows a Gaussian spatial dependence. The position of minimal frequency (i.e., temperature) corresponds to the middle point of the excitation spot. The frequency shift with respect to the outer edge of the excitation spot amounts to $0.5 \mathrm{GHz}$, similarly to the Kittel mode on continuous film. 
The Fourier amplitude of DE and Kittel modes is plotted in the top panel for comparison. As we can see, the fitted FWHM of DE amounts to $50-80 \mu \mathrm{m}$ for different scanning directions. The DE mode propagation would be evident as an asymmetric broadening with respect to the Kittel mode for scanning direction parallel to the expected DE wave vectors, i.e., perpendicular to the applied field (b) and at $45^{\circ}$ to the applied field (c). However, as we can see, in all three scanning directions DE-amplitude is contained within the Kittel mode amplitude, i.e., no significant broadening of DE-amplitude Gaussian compared to the Kittel mode is detected. This allows us to conclude that no propagation of DE mode can be detected within the experimental resolution. As the saturation magnetization in the pump spot increases towards the outer edge due to the increased temperature in the center, any spin wave traveling away from the excitation spot would have to propagate towards an increasing effective field. This means that the spin-wave frequency needs to be up-converted, in order to adapt to the local spin wave frequency, i.e., the spin waves would be continuously reflected back at the boundary with cooler region.

Moreover, optical spin-wave excitation is highly non-equilibrium ${ }^{163] 64}$, so that the resulting spin wave density is above the ballistic limit. This leads to high spin wave scattering probability and consequently, a reduced mean free path. 


\section{Chapter 5}

\section{Elastic dynamics in multilayer structures}

In this section we discuss elastic dynamics of multilayer systems, resolved by femtosecond pump-probe reflectivity measurements. We begin with a brief introduction of elastic dynamics in different multilayer systems with varying acoustic mismatch, as reported mainly in Ref. 80. Then, we focus on W/PC double-layers, with varying layer thickness and number of layers, that allow to control the excited elastic mode frequency. At last, the elastic dynamics in $\mathrm{CoFeB} / \mathrm{MgO}$ and $\mathrm{W} / \mathrm{PC}$ multilayers in two different pumping configurations are investigated: exciting elastic dynamics from the substrate (transmission geometry) and from the top layer (reflection geometry). Moreover, the role played by periodicity of the multilayer structures is investigated by comparing the results on periodic and aperiodic multilayers.

The reflectivity dynamics measurements reported in this chapter were performed by myself, with help from Henning Ulrichs. Finite-difference in time domain calculations of strain and reflectivity dynamics were developed by Henning Ulrichs, Dennis Meyer and Markus Müller.

\subsection{Introduction: the role of acoustic mismatch}

First of all, we illustrate the potential of the experimental setup for reflectivity dynamics in the following example measurement. Fig. 5.1 shows the reflectivity dynamics measured on a high-quality $31-\mathrm{nm} \mathrm{Bi}_{2} \mathrm{~T} e_{3}$ film, which structure properties have been described in section 3.3.3. The signal is dominated by a fast oscillation in the first 7 ps after the excitation and much slower dynamics at later times. The high-frequency, $1.84-\mathrm{THz}$, phonon mode can be traced back to a 540-fs dynamics of atoms moving in the most prominent optical $A_{1 \mathrm{~g}}$ 
mode 88899 . On the other hand, the slower mode matches a longitudinal phonon frequency, propagating through the film (with sound velocity $v_{l} \approx 4000 \mathrm{~m} / \mathrm{s}$ ). This way, we find a strong evidence that the experimental setup can simultaneously resolve phonons in $\mathrm{THz}$ frequency range, as well as slow elastic dynamics in $\mathrm{GHz}$ regime. Moreover, even the raw signal is very clear and can be interpreted without any further processing.

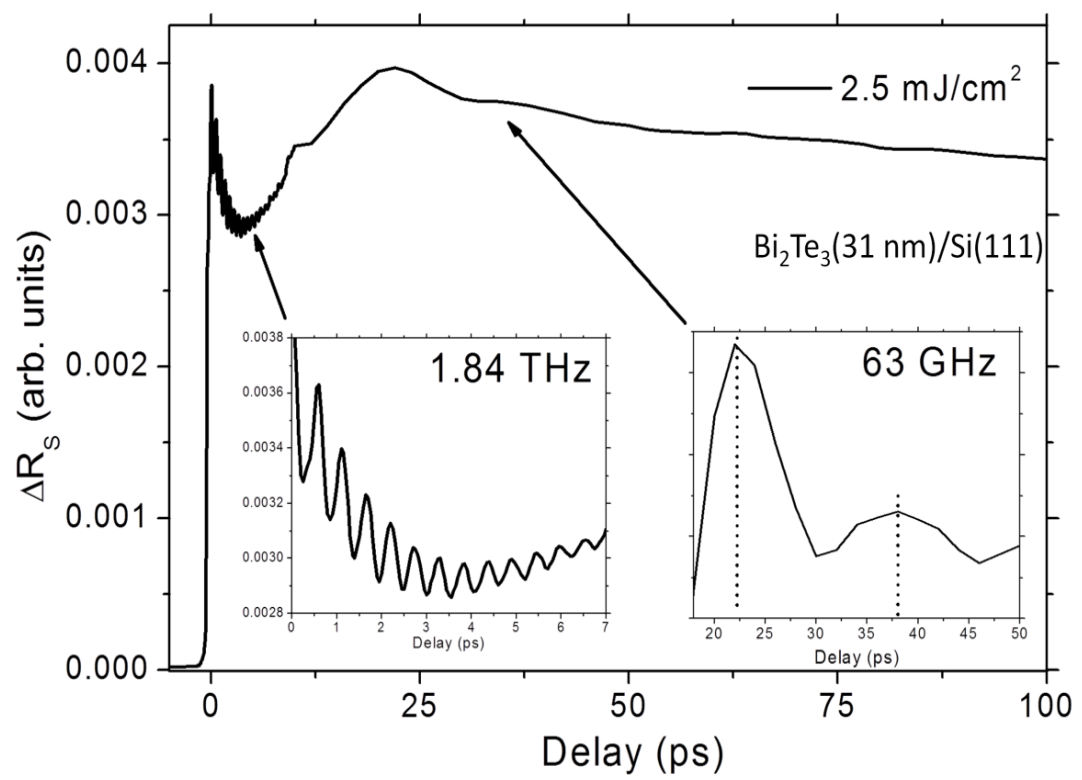

Figure 5.1: Reflectivity dynamics in a thermoelectric material, $31-\mathrm{nm} \mathrm{Bi} \mathrm{Te}_{3}$ film at $2.5 \mathrm{~mJ} / \mathrm{cm}^{2}$ laser fluence. The insets show two oscillation modes: a fast $1.84 \mathrm{THz}$ mode and a slower $63 \mathrm{GHz}$ mode ${ }^{90}$.

However, as we will see further through this chapter, the reflectivity signal from multilayer structures is much harder to interpret due to different, and often complex, layer contributions to the observed signal dynamics. Depending on the optical penetration depth in the material, typically only the top $\sim 20 \mathrm{~nm}$ in metals are sensed by the reflected probe beam. This characteristic length could span, in some cases, only the surface of bulk materials or the whole stack structure in a nano-scale multilayer. Thus, the support from numerical simulations of elastic dynamics is always helpful to interpret the experimental results.

In the rest of this section the first results of reflectivity dynamics on different materials are briefly addressed, and the role of acoustic mismatch between the adjoining soft/hard material layers is illustrated. As discussed in section 2.2 and shown in Fig 2.4, the reflection coefficient of an elastic wave, incident on a slab of soft material embedded between two hard layers, is close to 1 for practically all wave vectors, i.e., the soft layer serves as an acoustical isolator. This 
means that the band structure of such multilayer system will have large band gaps, forbidding the propagation of elastic waves, as shown in Fig 2.5.

On the other hand, structures with matching acoustic impedances (such as $\mathrm{CoFeB} / \mathrm{MgO}$ multilayers) will allow for modification of elastic wave dispersion characteristics (Fig. 2.6) with very few losses from back-reflections (Fig. 2.4).

Thus, there is a need for a collection of materials with a wide range of acoustic impedance values, that could be grown with sharp interfaces as discussed in section 3.3.2. In the following table we present the acoustic impedance of the relevant, investigated materials as calculated using Eq. 2.20:

\begin{tabular}{lcccccccr} 
Material & air & PC & glass & $\mathrm{Si}$ & $\mathrm{MgO}$ & $\mathrm{CoFeB}$ & $\mathrm{Cu}$ & $\mathrm{W}$ \\
\hline $\mathrm{Z}(\mathrm{mPa} * \mathrm{~s} / \mathrm{m})$ & 0.44 & 3 & 13 & 20 & 35 & 39 & 42 & 101
\end{tabular}

As we can see, a broad range of acoustic impedances are available, allowing to produce multilayers with high acoustic mismatch at the interfaces, such as $\mathrm{W} / \mathrm{PC}, \mathrm{Cu} / \mathrm{PC}$ or $\mathrm{W} / \mathrm{Si}$. For comparison, also metal/oxide multilayers with a low acoustic mismatch, namely $\mathrm{CoFeb} / \mathrm{MgO}$, were produced. Different material combinations can provide not only the control over the acoustic mismatch, but ultimately, manipulate the energy relaxation channels. For example, in metals the energy is first deposited into the electronic bath, however in electric isolators such as $\mathrm{MgO}, \mathrm{Si}$, and $\mathrm{PC}$ only phonons are available for energy relaxation.

Reflectivity dynamics in a $\mathrm{Cu} / \mathrm{PC}$ double layer were measured as shown in Fig. 5.2, where a strong $f_{0}=38 \mathrm{GHz}$ oscillation response can be clearly seen. To understand whether this oscillation corresponds to a localized or extended mode, a FTDT simulation was performed, applying a sinusoidal stress wave with $f_{0}$ frequency on the surface and calculating the amplitude of system response as function of depth $z$. This is shown in Fig. 5.2 (c), where the excited mode can be associated to a standing wave localized in the thick PC layer. In the same way, an aperiodic $\mathrm{FePtAgCu} / \mathrm{NiTa}$ sample, used in magnetic recording, was investigated, obtaining similar results albeit showing a higher frequency mode due to the reduced total thickness of the sample.

The influence of acoustic mismatch was clarified by reflectivity dynamics shown in Fig. 5.3. As we can see, in the $\mathrm{CoFeB} / \mathrm{MgO}$ multilayer an elastic wave is excited through the stack, due to the similar acoustic impedance of each layer, with a frequency that lies below the band gap. Moreover, the periodicity of the elastic response is given by the periodicity of the multilayer stacking and layer thicknesses. This way, it is reasonable to argue that higher oscillation frequencies can be reached by decreasing the individual layer thickness. More importantly, as we can see in the lower panel of Fig. 5.3. only the very first layer of $\mathrm{W} / \mathrm{Si}$ multilayer oscillates when a strain is applied with the most prominent frequency obtained in the Fourier spectrum of measured reflectivity dynamics. The mode is strongly damped into the depth of the film: i.e., the simulations 
(a)

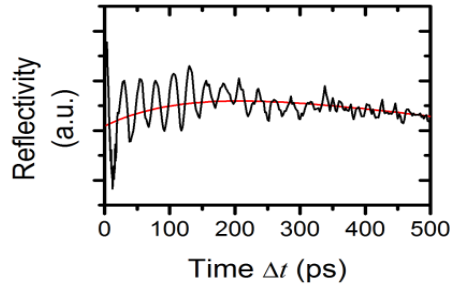

(d)

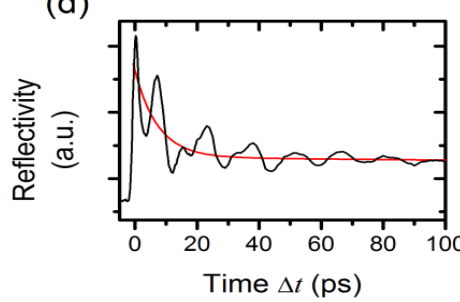

(b)

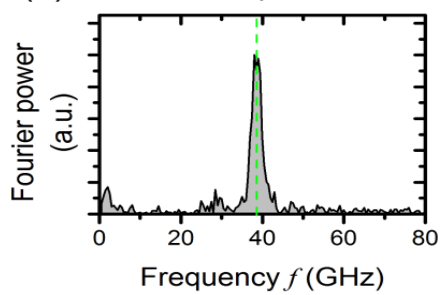

(e)

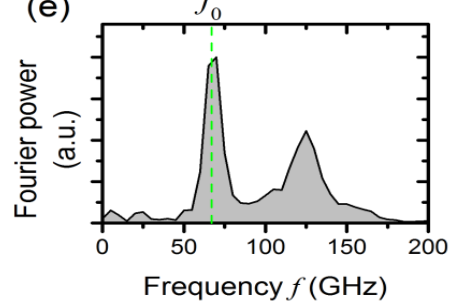

(c)

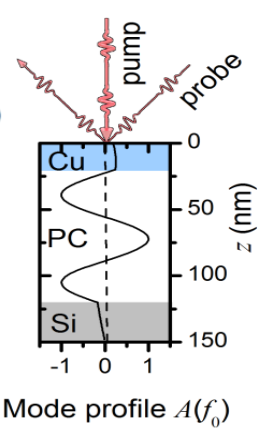

(f)

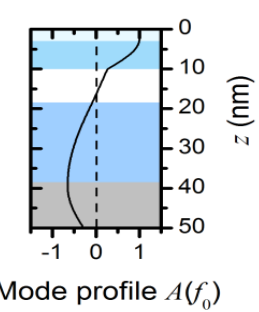

Figure 5.2: a) Time-resolved reflectivity dynamics on $20 \mathrm{~nm} \mathrm{Cu} / 100 \mathrm{~nm} \mathrm{PC}$ double layer. The decaying background of incoherent heating has been removed. (b) Fourier power spectrum of the reflectivity oscillations with a localized standing phonon mode at $f_{0}=38 \mathrm{GHz}$ in the $100 \mathrm{~nm}$ PC layer. (c) Sketch of the pump-probe reflectivity experiment on the $\mathrm{Cu} / \mathrm{PC}$ bilayer on a Si substrate together with the mode profile determined by a FDTD simulation. (d-f) Same graphs for the HAMR FePtAgCu/NiTa sample. $\stackrel{80}{[}$

suggest that the high acoustic mismatch prevents the elastic mode from propagating further down into the sample by continuously reflecting the incoming stress pulse at each interface.

After this brief introduction, in the rest of this work mainly W/PC structures are discussed, since their acoustic mismatch is even higher than in W/Si layers. Furthermore, there is a need to experimentally distinguish whether an elastic mode is a collective oscillation of the whole stack or a damped oscillation near the surface. This cannot be deduced from the Fourier spectrum of reflectivity signal alone, since only the surface is probed, exactly on the same spot where the elastic dynamics is excited. From the experimental point of view, this information can be retrieved by working in different excitation and probing geometries: probing the dynamics on the front (free) surface of the sample while exciting the elastic dynamics from the opposite (substrate) side. Once the possibility of exciting high-frequency elastic modes has been established above, band gap tuning and the role of aperiodicity of the multilayer stack in the blocking of the elastic mode can be addressed. 

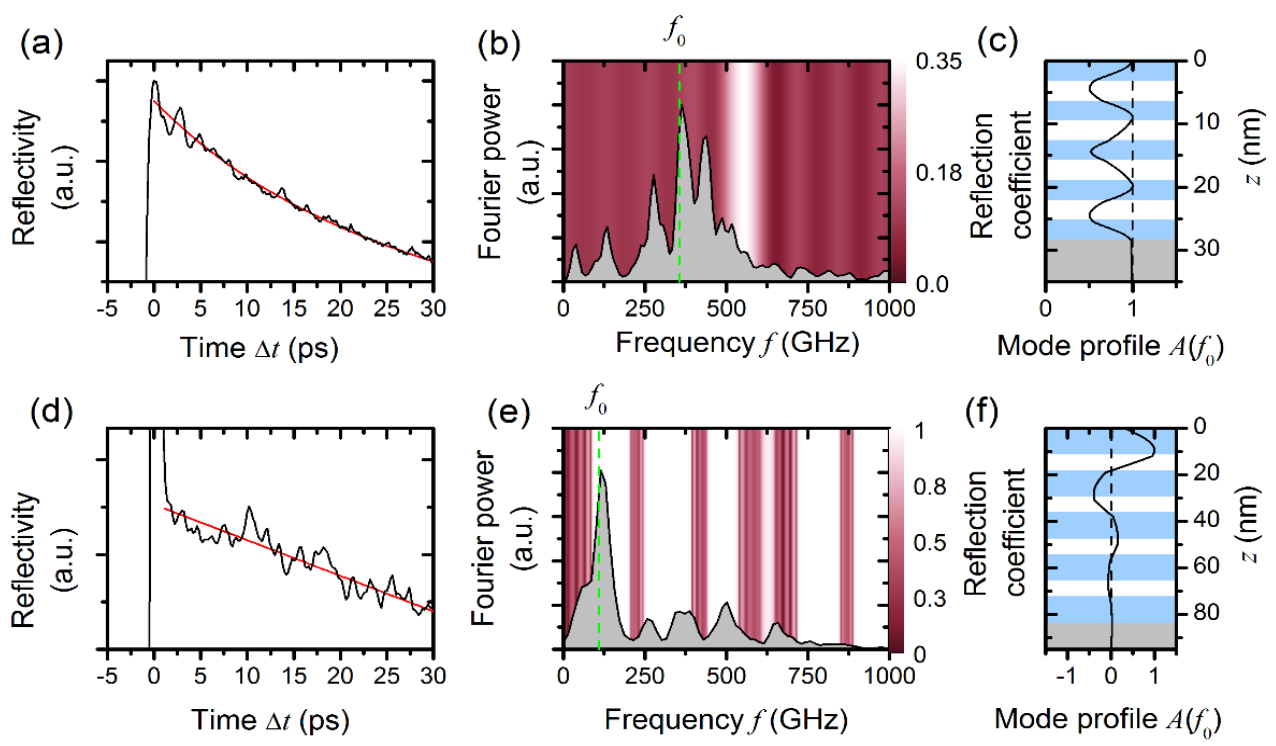

Figure 5.3: Time-resolved reflectivity measurement on (a) a $3.15 \mathrm{~nm} \mathrm{CoFeB/}$ $3.15 \mathrm{~nm} \mathrm{MgO}$ multilayer sample. The incoherent background (red) was removed and (b) Fourier spectrum calculated. (c) FDTD calculation of the inplane mode profile showing a localized standing wave within the layer structure. (d) Reflectivity dynamics of a $12 \mathrm{~nm} \mathrm{W/} 7 \mathrm{~nm}$ Si multilayer sample with (e) corresponding Fourier power (f) a mode profile of sagittal wave with a damped oscillation inside the multilayer due to the high acoustic mismatch. $\frac{80}{}$

\section{$5.2 \quad$ W/PC multilayer structures}

In this section we present a systematic study of elastic dynamics in tungsten (W) and polycarbonate (PC) multilayer structures. First, single tungsten layers are addressed. Later on, elastic dynamics on double layer of W/PC of different thicknesses are analyzed. This gives a general overview of excitation mechanism and signal interpretation in these structures. Moreover, some material properties can be deduced, which will be helpful for the interpretation of more complicated structures and in numerical simulations discussed later on. In all experiments of this section, the dynamics is excited and probed on the front (free) surface of the film.

\subsubsection{Single $W$ layers: general considerations}

In Fig. 5.4a the reflectivity dynamics obtained on a single $200 \mathrm{~nm}$ tungsten film on a thick Si substrate is shown. At the arrival of pump pulse (around $0 \mathrm{ps}$ ) 


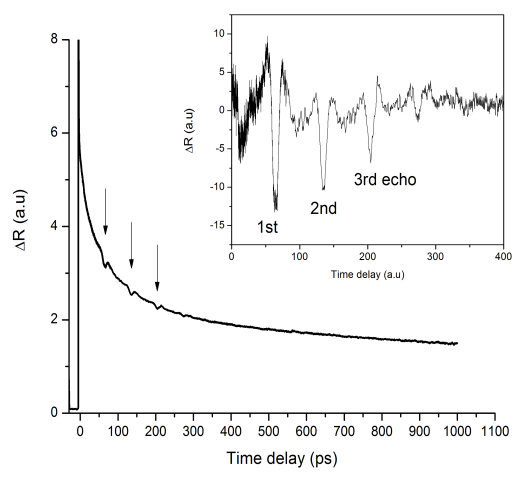

(a)

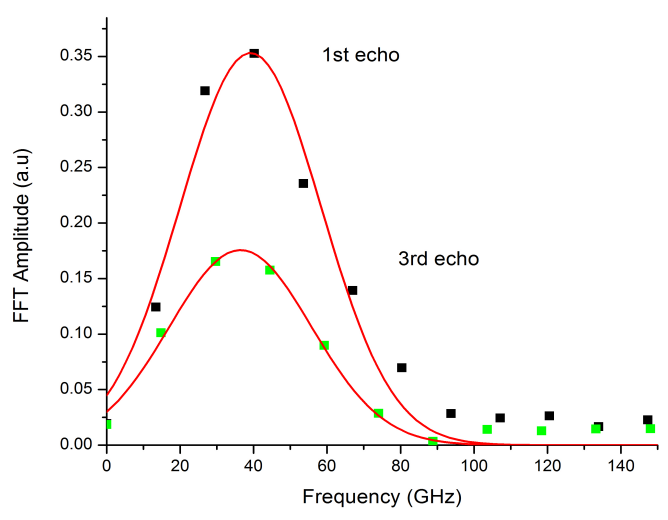

(b)

Figure 5.4: a) Reflectivity dynamics on a 200-nm tungsten layer on Si substrate where excited acoustic echoes can be observed (indicated by arrows). The inset shows the echoes after incoherent background subtraction. b) FFT amplitude of reflectivity dynamics signal in a 100 ps time window around 1st (black squares) and 3rd (green squares) echoes. A bandwidth of $\approx 39 \mathrm{GHz}$ can be estimated from the fitted Gaussian curves (red line). The reflection coefficient can be estimated as the amplitude ratio of two successive echoes to $r_{W / S i} \approx 0.7$

we can see a fast change in reflectivity, caused by a change in density of states due to photoexcited electrons that modify the material optical constants. After a fast electron thermalization $(\approx 100 \mathrm{fs})$ on the surface, the energy is transferred to the phonons reservoir $(1-2 \mathrm{ps})$, launching phonon modes and, on a longer time scale, acoustic modes ( $>100 \mathrm{ps}$ ) at a constant temperature. Heat diffusion takes place on a longer timescale ( $\gg 1 \mathrm{~ns})$. This is shown as an incoherent background decay towards the initial (non-perturbed) state and an additional pulse-like change in reflectivity signal (see inset). These are the strain pulses, launched by the fast thermal expansion of the material, traveling perpendicularly to the sample plane and back after the reflection on Si substrate.

Total deposited energy per unit volume $W$ as function of the distance to film surface $z$ can be calculated following the equation 2.26. Moreover, dividing by the specific heat per unit volume $\mathrm{C}=2.58 \mathrm{~J} / \mathrm{cm}^{3} \mathrm{~K}$ (for tungsten) we obtain the temperature profile induced by laser heating,

$$
\Delta \mathrm{T}(z)=\mathrm{W}(z) / \mathrm{C}
$$

that allows us to estimate a maximum change in temperature at the film surface $\mathrm{T} \approx 800 \mathrm{~K}$. The temperature profile in the sample follows the exponential profile of optical penetration in the opaque material. The absorbed energy initiates a thermal expansion in the film and, consequently, produces a strain 
pulse. Its initial value on the film surface can be calculated using bulk modulus $\mathrm{B}=310 \mathrm{GPa}$, and linear expansion coefficient $\beta=4.5 \mu \mathrm{m} / \mathrm{m} * \mathrm{~K}$ (for tungsten) as $\sigma_{\text {thermal }}=-3 \mathrm{~B} \beta \Delta \mathrm{T}(z) \approx 3.4 \mathrm{GPa}$.

The strain pulse travels perpendicular to the surface plane of the sample and, after bouncing back on the substrate, it is detected as a change in reflected intensity (see inset in Fig. 5.4a). This way, the time between consecutive strain pulses can be estimated $\approx 72 \mathrm{ps}$ and the traveled distance $\approx 400 \mathrm{~nm}$, so that the sound velocity in tungsten film is calculated $\nu_{W, 200 \mathrm{~nm}} \approx 5520 \mathrm{~m} / \mathrm{s}$, which is slightly above the longitudinal sound velocity in bulk $v=5280 \mathrm{~m} / \mathrm{s}$.

Optical penetration depth in tungsten film defines the bandwidth of the excited strain pulse. We can estimate the spectral content of a strain pulse following Eq. 2.30, where the excitation bandwidth is given by $v / \zeta \approx 39 \mathrm{GHz}$. On the other hand, the spectral content of the echoes can be estimated also from the experimental data as the width of Fourier transform of each echo. As shown in Fig. $5.4 \mathrm{~b}$ the width of the Gaussian fitted to Fourier transform amplitude of echoes amounts to $\approx 39 \mathrm{GHz}$, in perfect agreement to the expected values. The refection coefficient can be estimated from the experimental data as the ratio between the amplitudes of two consecutive echoes. From the data shown in Fig. 5.4b we can calculate $r_{W / S i} \approx 0.69$. We can also estimate the expected reflection coefficient for $\mathrm{W} / \mathrm{Si}$ interface following Eq. $2.19 \mathrm{r}_{\mathrm{W} / \mathrm{Si}} \approx 0.67$, in good agreement with experimentally determined value.

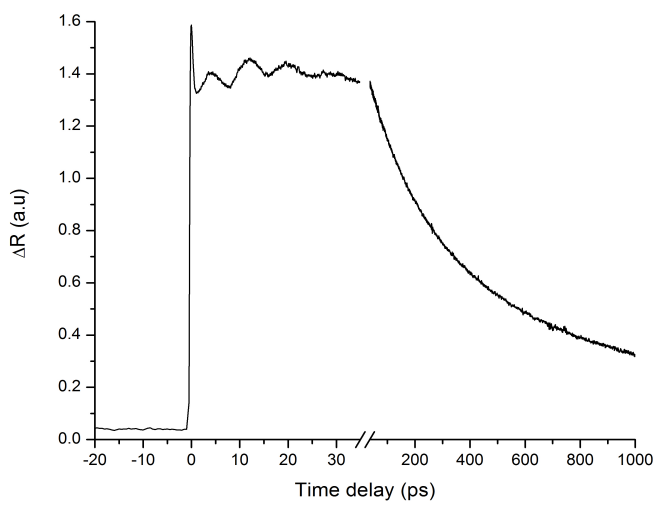

(a)

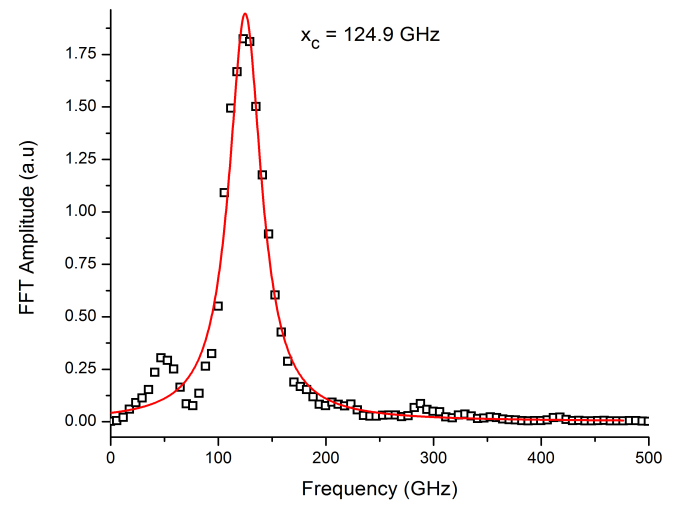

(b)

Figure 5.5: a) Time-resolved reflectivity dynamics on 21.6-nm tungsten layer. Fast oscillations are seen in the first $35 \mathrm{ps}$. b) FFT transform of the oscillations shows a peak at $x_{c}=124.9 \mathrm{GHz}$ central frequency. Red line is a Lorentzian fit to the data.

When the film thickness becomes comparable to the optical penetration depth in tungsten, the whole film is probed (and excited) almost homogeneously. The 
resulting reflectivity dynamics signal carries not only the information about the strain dynamics on the surface, but the integration over the whole film volume. As a result, rather an oscillation instead of strain pulse is observed (see Fig. 5.5a). Interestingly, these oscillations vanish after 4 periods (reflections on the substrate), analogously to the detected 4 signal pulses in the much thicker (200 nm) sample. This suggests that the main losses in signal amplitude come from strain pulse being partially transmitted into the substrate. Thus, a substrate with lower acoustic impedance, such as glass, would be better suited for the experiments and was implemented in later samples.

It's also interesting to consider the Fourier-transformed reflectivity dynamics signal in this sample. Fig. 5.5b shows the FFT amplitude of the first 30 ps of the reflectivity signal on $21.6 \mathrm{~nm} \mathrm{~W}$ layer. A strong frequency peak is observed at $124.9 \mathrm{GHz}$, that would yield a sound velocity $v_{W, 21.6 \mathrm{~nm}}=5395 \mathrm{~m} / \mathrm{s}$, in good agreement (2\% deviation) to the sound velocity calculated for $200 \mathrm{~nm}$ - thick sample. Small differences might be expected due to deviations of the thickness from nominal values. In the calculation above a wavelength corresponding to twice the sample thickness was supposed, since $Z_{W} / Z_{S i}>1$ and $Z_{W} / Z_{\text {air }}>1$ even orders of half-wavelength should be expected, as in case of a displacement profile in a open-ends pipe geometry.

\subsubsection{W/PC double layers}

In this section, double layers of tungsten deposited on top of polycarbonate films on silicon substrate are considered. In contrast to tungsten, PC is a disordered polymer, almost transparent $(\mathrm{R}=0.1)$ to infrared light. Moreover, as previously noted, this is a soft material, so that the sound velocity in PC amounts to $v_{P C}=2300 \mathrm{~m} / \mathrm{s}$ and its acoustic impedance is very low ${ }^{91}$. Several $\mathrm{W} / \mathrm{PC}$ double layers were fabricated: tungsten thickness was systematically increased until reaching $100 \mathrm{~nm}$, while the PC layer thickness was maintained relatively constant, at $\approx 150 \mathrm{~nm}$. This will allow us to establish the effect of the soft PC layer inserted between the hard W and Si layers, by comparing the result to the previous section.

As we can see in Fig. 5.6, these samples have a rich elastic dynamics. In the $5.6 \mathrm{~nm} \mathrm{~W} / 164 \mathrm{~nm}$ PC double layer, a long timescale, low-frequency oscillation is observed. This frequency must belong to the elastic dynamics in the PC layer: as shown in the previous section, oscillations in $\mathrm{W}$ are expected to have much higher frequencies. However, since PC is practically transparent to infrared light, the dynamics must be launched by the thermal expansion of thin W layer, so that it acts as a transducer. In effect, when we consider that the excited frequency amounts to $13.8 \mathrm{GHz}$ (as shown in the upper panel of Fig. 


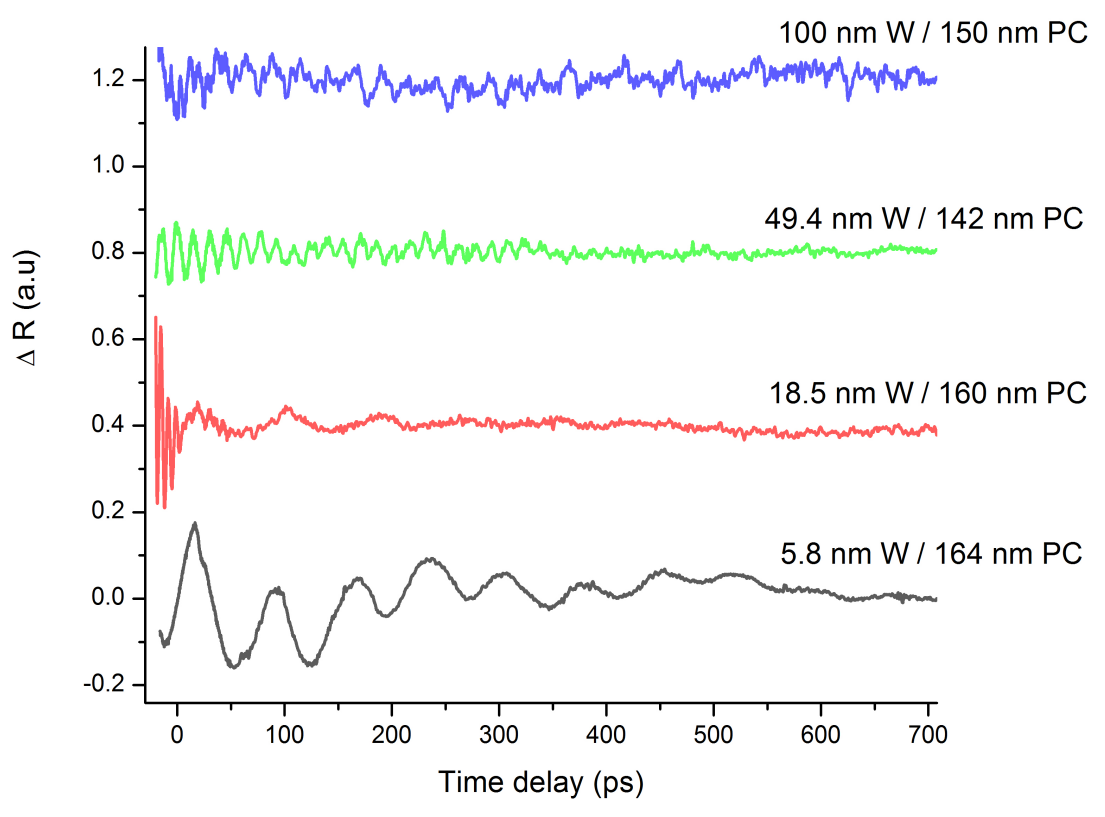

Figure 5.6: Time-resolved reflectivity measurement in W/PC double layers on Si substrate. Incoherent background has been subtracted. Each dataset is offset by 0.4 a.u. The legends indicate the respective tungsten $(\mathrm{W})$ and polycarbonate (PC) thicknesses.

5.7), and the PC layer thickness is $164 \mathrm{~nm}$, we can calculate a sound velocity $v_{\mathrm{PC}, \text { calc }}=2263 \mathrm{~m} / \mathrm{s}$, slightly below the literature value for PC.

As $\mathrm{W}$ film thickness increases, we can observe fast elastic dynamics in the first $50 \mathrm{ps}$ (see for example red curve in Fig. 5.6) and a trace of long timescale oscillation later on, that are suppressed with increasing W thickness. Moreover, as can be seen in the FFT of the reflectivity signal (shown in Fig. 5.7) the peak at low frequencies $\leqslant 30 \mathrm{GHz}$ (in upper panels) decreases its amplitude with increasing $\mathrm{W}$ thickness (lower panels). This hints that the elastic dynamics in the PC layer is not effectively probed, when tungsten thickness becomes comparable to the optical penetration depth. On the other hand, the high-frequency peak can be associated to the elastic dynamics in the tungsten layer, since its frequency shifts inversely proportional to the layer thickness.

This behavior can be understood in terms of resonant frequencies of the soft PC layer, excited by the tungsten transducer and the coupling between then. The PC layer is sandwiched between the hard Si and W layers, so that the displacement on the $\mathrm{W} / \mathrm{PC}$ and $\mathrm{PC} / \mathrm{Si}$ interfaces must be zero. The strain then must be maximum at the interface, in analogy to a both-ends closed cavity. 

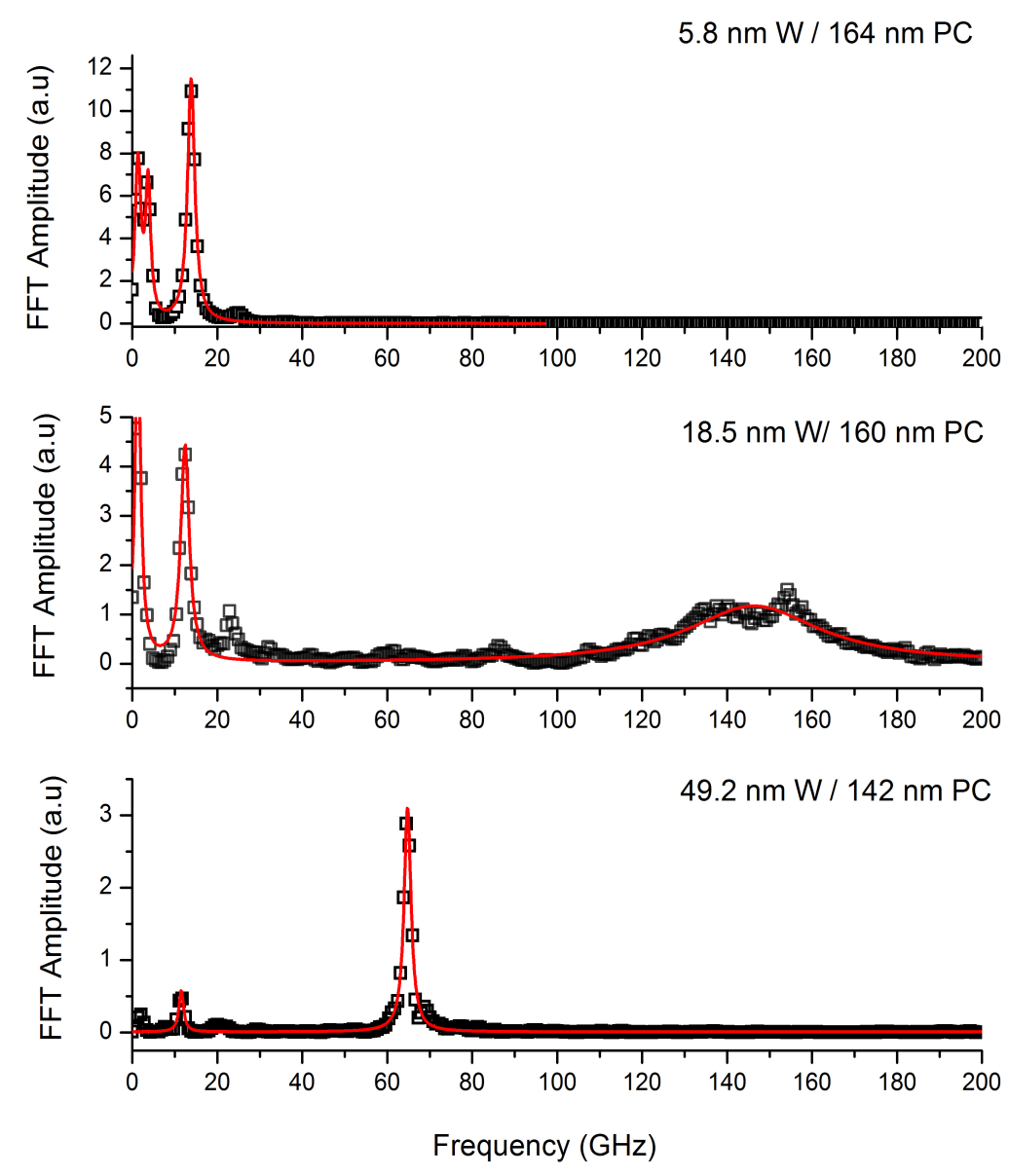

Figure 5.7: FFT amplitude of correlated reflectivity dynamics on W/PC double layers (shown in Fig. 5.6). Red line is a Lorentzian fit to the data. Legends above each graph indicate the respective thickness of tungsten $(\mathrm{W})$ and polycarbonate (PC) layers.

Then, the natural frequencies of this cavity are given by:

$$
f_{n}=n \frac{v}{2 d}
$$

where $n=1,2,3 . ., v$ is the sound velocity and $d$ is the film thickness. If we suppose that the PC thickness is kept constant at $150 \mathrm{~nm}$, we can calculate the separation between the natural modes: $\Delta(\mathrm{f})=9.9 \mathrm{GHz}$. This value will slightly differ for each sample, since the PC thickness is not exactly $150 \mathrm{~nm}$ in all of them. However, as we can see in Fig. $5.8(\mathrm{a}-\mathrm{c})$ this is indeed the case, PC layer presents a comb of strain frequencies. 


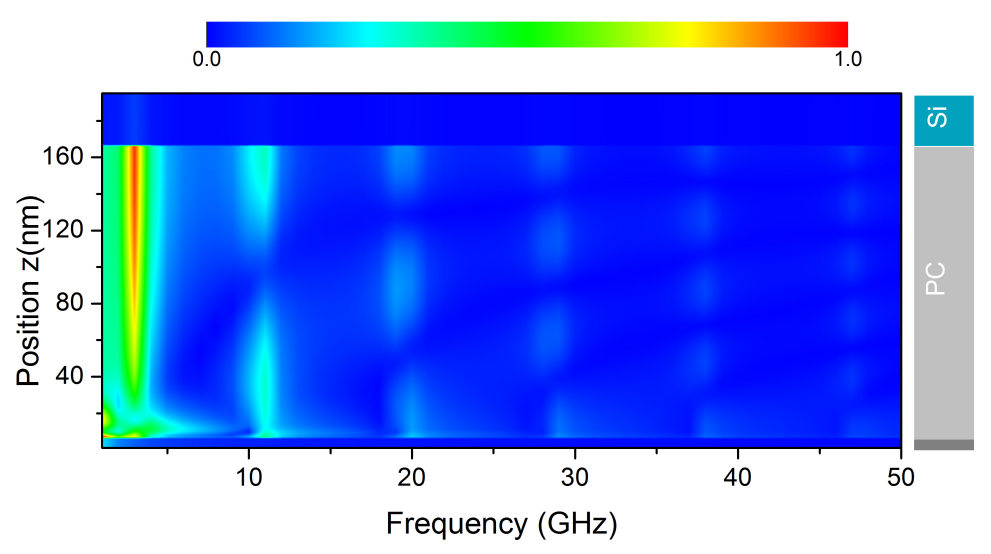

(a) $6 \mathrm{~nm} W / 169 \mathrm{~nm}$ PC

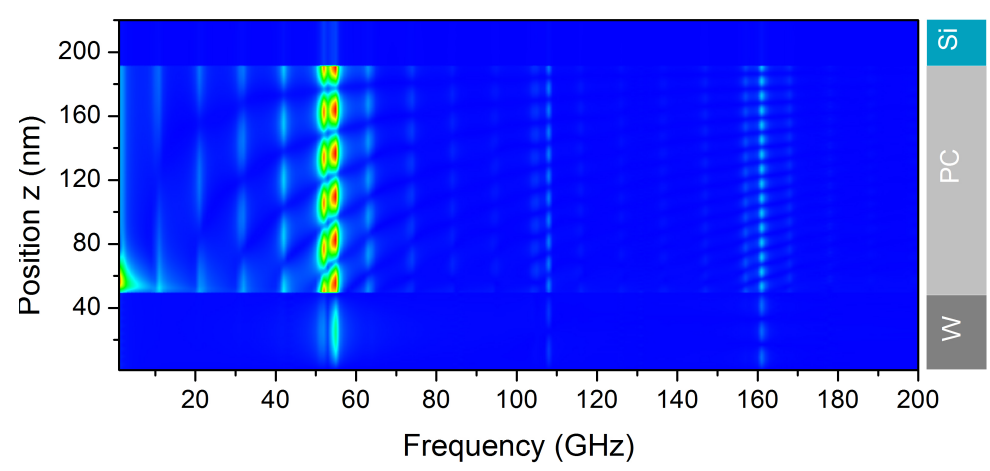

(b) $49 \mathrm{~nm} \mathrm{W/142} \mathrm{nm} \mathrm{PC}$

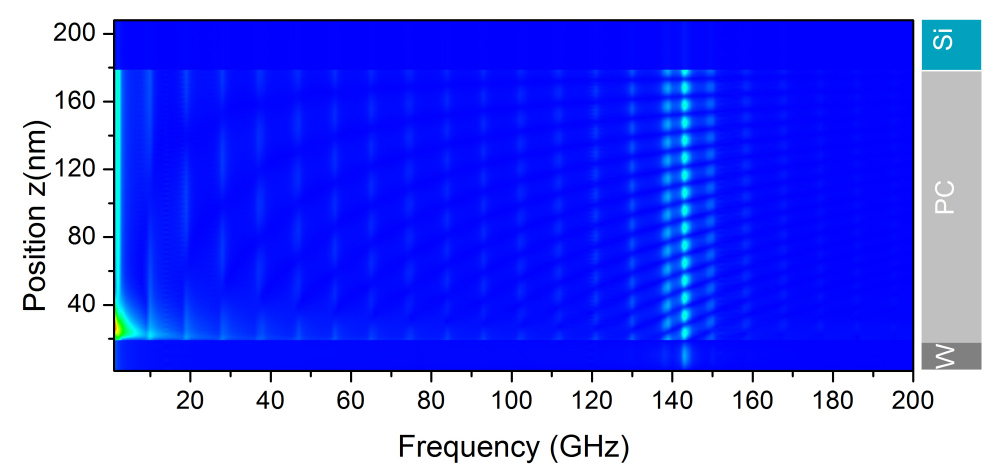

(c) $18.5 \mathrm{~nm} \mathrm{~W} / 160 \mathrm{~nm}$ PC

Figure 5.8: FFT amplitude of simulated elastic strain (in color code and normalized) in W/PC double layers on Si substrate as function of depth z. Drawings on the right indicate the multilayer structure. 
The function of the W-layer is to select resonantly excited frequencies in the soft and transparent PC. The central frequency of excitation is defined by the W thickness and, in contrast to PC layer, we can think of it as a open-end cavity, because the hard $\mathrm{W}$ layer can almost freely displace into the soft air and PC. This way, the boundary conditions at the interface is maximum displacement and minimum pressure, accommodating multiples of a half-wavelength between the interfaces. As we can see in Fig. $5.8(b, c)$ this is indeed the case for the $\mathrm{W}$-layer with the maximum strain at around $142 \mathrm{GHz}$ (b) and $55 \mathrm{GHz}$ (c). On the other hand, the excitation bandwidth is given by the spectral width of the strain pulse in the $\mathrm{W}$-layer, which is $\approx 39 \mathrm{GHz}$, as we have previously seen in Fig. 5.4b. Since the excitation bandwidth is greater than the natural frequency separation between the modes in PC layer, an effective coupling into this layer is expected at frequencies $\leqslant 39 \mathrm{GHz}$. Indeed, this is seen in experimental data Fig. 5.7, where the lower PC-cavity modes are excited.

When the W-layer is thinner than the optical penetration length, as for example, in the sample with a $5-\mathrm{nm} \mathrm{W}$ layer on top, the excitation bandwidth will be given not by the the spectral content of the strain pulse, but rather by W-layer thickness and the strain profile therein, yielding a broader bandwidth. This will be relevant for the structures with thin W-layers, where each one would act as a broadband source of elastic excitations.

\subsubsection{W/PC multilayers}

In this section we address the elastic dynamics in W/PC multilayer structures. One of the most interesting aspects is whether a collective oscillation mode of the whole stack can be excited, instead of the individual modes in each layer. Namely, three periodic W/PC multilayer samples with varying layer thickness and stack repetition have been considered. The total multilayer thickness was maintained approximately constant, $72-76 \mathrm{~nm}$. This means thicker $\mathrm{W}$ layers are associated with fewer stack repetitions.

As shown in Fig. 5.9 all multilayers present a fast oscillation dynamics in the first 30 ps after the excitation, and a slower dynamics at later times, similar to $18.5 \mathrm{~nm} \mathrm{W/} 100 \mathrm{~nm}$ PC double layer presented in the previous section. Moreover, as shown in Fig. 5.10. FFT spectra of elastic dynamics in the multilayer samples do not show any obvious dependence on the layer thickness neither in low- (right) or high-frequency (left) regimes. Oscillation frequencies of the single PC layer with the same thickness as in the multilayers lie above 50 $\mathrm{GHz}$, and single W-layer oscillations do not match the observed high frequencies. This way, no single layer can be associated with the oscillation frequencies in these multilayers. 


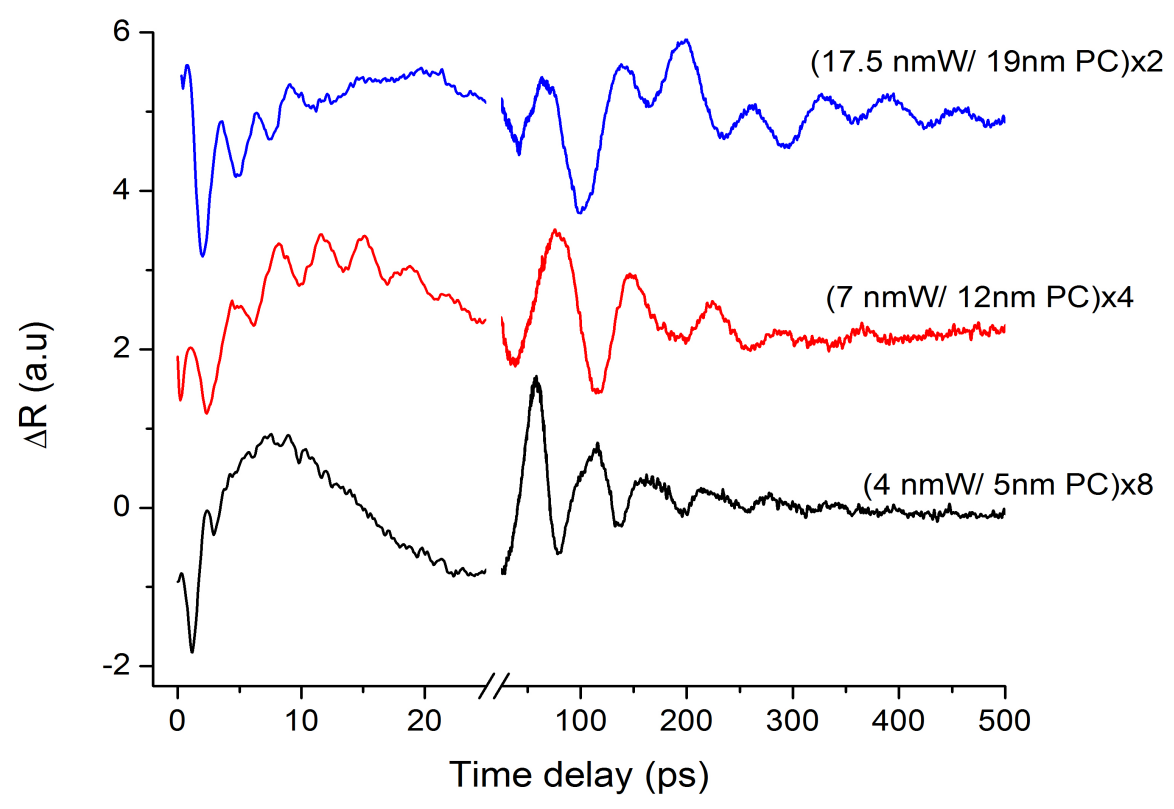

Figure 5.9: Reflectivity dynamics on W/PC multilayer structures. Incoherent background has been subtracted. A 2 a.u offset is inserted between the datasets for better visibility. Legends indicate the thickness of tungsten $(\mathrm{W})$ and polycarbonate (PC) layers and number of stack repetitions. Fast reflectivity dynamics are observed in the first $\approx 30 \mathrm{ps}$ and slower oscillations during the rest of the signal.

As we can see in Fig. 5.10b and 5.10a, there is very little change in the detected frequencies, even though the PC and W-layer thicknesses were drastically decreased. This suggests that W and PC layers are not acoustically isolated anymore, but exhibit a rather collective behavior. Taking into account the calculated dispersion relations for infinite multilayer system shown in Fig. 2.5, we can see that the low-frequency modes belong to the first acoustic band below $50 \mathrm{GHz}$. Due to the finite number of stacks in the multilayer, only some bulk modes are allowed, whose frequencies are given by Eq. 2.25 and indicated by red dots in Fig. 5.10b. Thus, there is a qualitative agreement with the expected frequencies. Moreover, the high-frequency modes would lie in the band gap, suggesting that they belong to highly damped mode localized near the surface. This also explains the short lifetime of this mode, below 30 ps. 


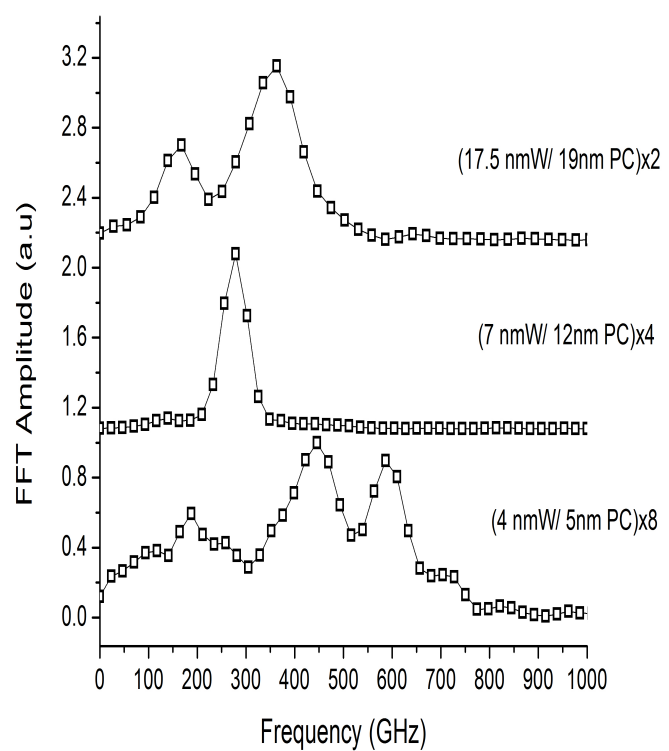

(a)

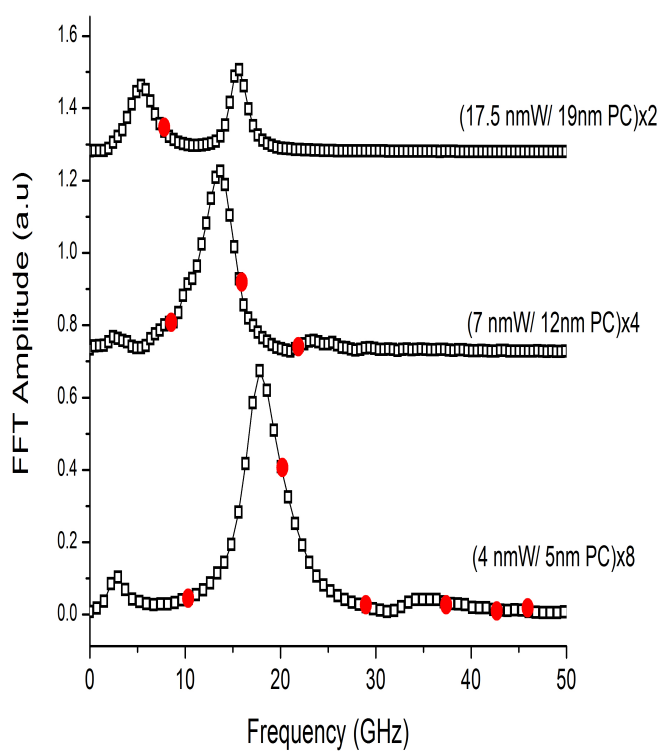

(b)

Figure 5.10: Correlated FFT amplitude (a) in the first 30 ps dynamics and (b) the rest of the elastic dynamics in multilayer W/PC structures shown in Fig. 5.9. Red dots indicate the frequencies of the bulk mode, calculated using the dispersion relation shown in Fig. 2.5 and Eq. 2.25. Legends indicate tungsten (W) and polycarbonate (PC) thicknesses and the number of stack repetitions. Data is offset for better visibility.

\subsection{Elastic dynamics in periodic and aperiodic struc- tures with different pumping orientations}

In this section we introduce the elastic dynamics of periodic and aperiodic structures in two different excitation geometries. The most straightforward way of exciting and detecting reflectivity dynamics in our setup is setting the pump and probe beams on the same side of the sample, i.e. the reflection geometry. However, in order to address the blocking of elastic waves through the multilayer stack, the transmission geometry also needs to be considered, i.e., exciting elastic dynamics on the opposite (substrate) side of the multilayer structure.

When the multilayer structure is periodic, same elastic modes should be expected in the sample, regardless of the excitation geometry. As shown in Fig. 5.12a, this is indeed the case: both excitation geometries show the same two frequency peaks, although with different relative amplitudes. In particular, it is noteworthy that the frequency peak at $\sim 0.55 \mathrm{THz}$ was detected, the highest oscillation frequency in the multilayers investigated so far, with a large ampli- 
tude.

Comparing with the calculated dispersion relation of a CoFeb/MgO multilayer shown in Fig. 2.6 (black line) we can see that this elastic mode corresponds to a frequency excited at the band gap in Brillouin zone edge, at $k D=0$. The other frequency mode, at $\sim 100 \mathrm{GHz}$, corresponds roughly to the middle of Brillouin zone. Again, as in the case of W/PC multilayers discussed in the previous section, these are a high-frequency surface mode with an exponentially decaying amplitude profile and a low-frequency bulk mode.

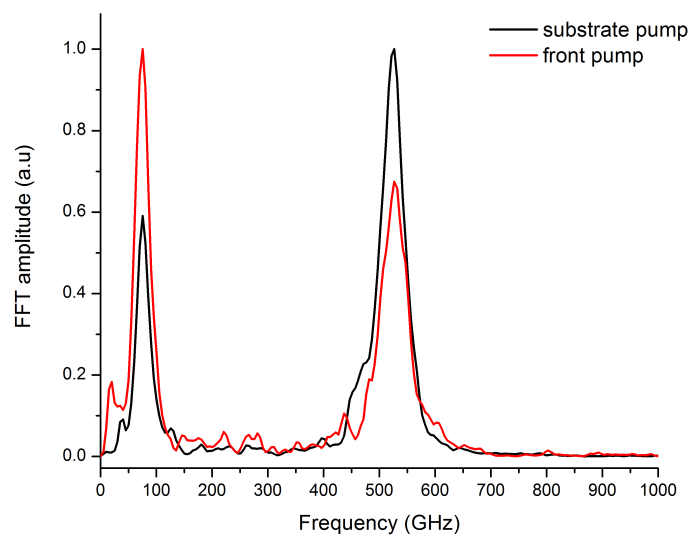

(a)

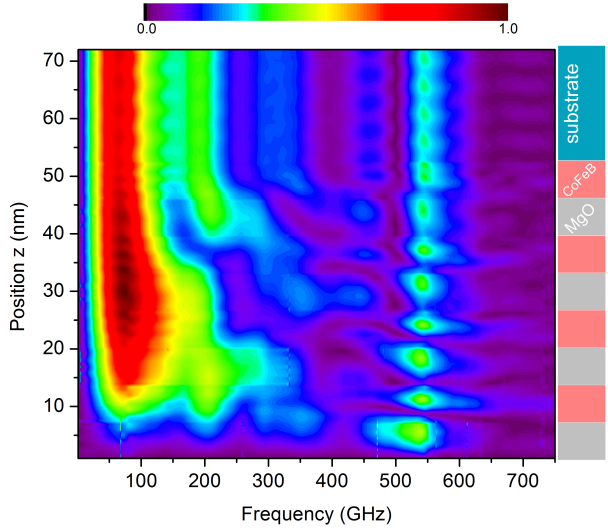

(b)

Figure 5.11: a) Normalized FFT amplitude of correlated reflectivity dynamics on $\mathrm{CoFeB} / \mathrm{MgO}$ multilayer with periodic stacking after incoherent-background subtraction. b) FFT amplitude of simulated elastic stress as function of depth for front pump orientation. The periodic multilayer consists of a $(6.5 / \mathrm{nm} / \mathrm{CoFeB} / 6.5 / \mathrm{nm} / \mathrm{MgO}) \times 4$ stack on $\mathrm{MgO}$ substrate. Two excitation geometries are considered: from the substrate (black) and front side (red), while always probing the front side.

As shown in the elastic dynamics simulations (Fig. 5.11b), indeed two such modes are present. The high-frequency mode has a maximum amplitude localized in each $\mathrm{CoFeB}$ layer, because the $\mathrm{MgO}$ layers do not absorb the pump pulse. This way, the optical absorption favors the excitation of elastic waves with frequencies in the second band gap. The amplitude of the surface mode is highly reduced compared to the bulk mode. Moreover, it decays with increasing depth into the sample, as expected, although a complete extinguishing of the elastic mode is not achieved due to the finite number of stack repetitions, i.e., the wavelength is comparable to the total multilayer thickness.

Additionally, an aperiodic $\mathrm{CoFeB} / \mathrm{MgO}$ sample with the same total thickness $(\approx 52 \mathrm{~nm})$ as the periodic one was fabricated. In this case, there is no 
proportionality factor between the successive layers of $\mathrm{CoFeB}$ (or $\mathrm{MgO}$ ) layers, but prime-number thicknesses are used (3,5,7,11 nm). As shown in Fig. 5.12b. high-frequency peak is suppressed for both excitation schemes (back and front pumping), in the sense that it has a much lower amplitude than the bulk-like mode. This suppression seems more efficient when pumping thin layers first (front scheme). This result indicates that aperiodic multilayer structure hinders the excitation of the high-frequency elastic modes. Moreover, at low frequencies the dominating elastic mode is the same as for the periodic structure, in agreement to its bulk-like behavior.

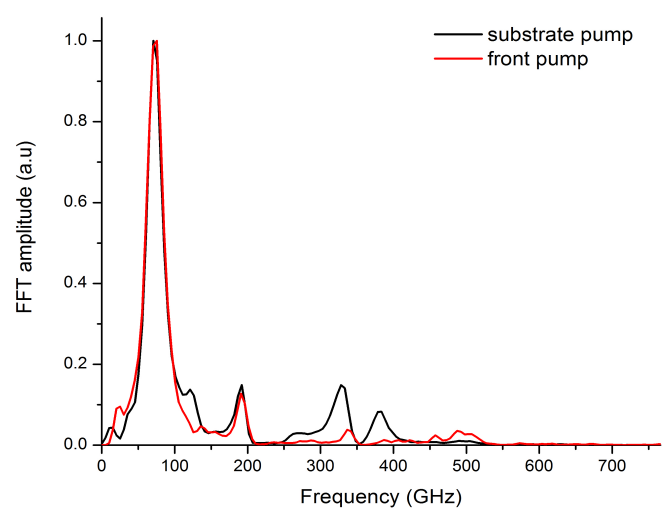

(a)

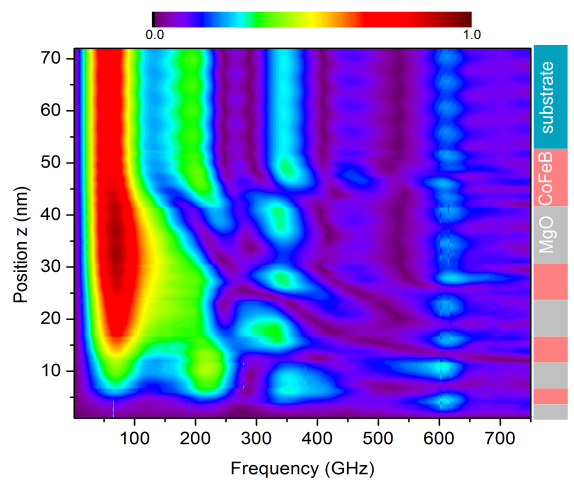

(b)

Figure 5.12: a) Normalized FFT amplitude of correlated reflectivity dynamics on the aperiodic $\mathrm{CoFeB} / \mathrm{MgO}$ multilayer after subtracting the incoherent background. b) FFT amplitude of simulated elastic stress as function of depth for front pump orientation. The aperiodic sample is namely $(x \mathrm{~nm} \mathrm{CoFeB} / x \mathrm{~nm} \mathrm{MgO}$ ) with $x \in(3,5,7,11 \mathrm{~nm}$ ) multilayer stack on $\mathrm{MgO}$ substrate. Two excitation geometries are considered: from the substrate (black) and front side (red), while always probing the front side.

In order to further develop these ideas, a non-commensurate $\mathrm{W} / \mathrm{PC}$ multilayer was fabricated. In contrast to the previous sample, where PC layer thickness is the same as the adjacent $W$ layer, each layer in the sample has different thickness. Two such samples with inverse stacking order were fabricated. In both cases, a 9.6-nm W layer was added at the bottom (substrate side), that acts as a transducer.

As shown in Fig. 5.13a, different modes are detected on the front side of the sample depending on the orientation of the pump. Moreover, these modes are complementary: when pumping from the substrate, a large amplitude mode at $\approx 14 \mathrm{GHz}$ is detected together with two side frequencies and when pumping from the front this mode is suppressed, while the two side modes grow in am- 
plitude. Analogously to the W/PC periodic multilayers, none of the detected frequencies can be explained as an oscillation of a single layer. For example, the thickest layer of PC is $21.8 \mathrm{~nm}$, that would yield a first mode frequency of $\approx 68 \mathrm{GH} z$ and the $\mathrm{W}$ layers yield even higher frequencies due to a higher sound velocity in them.

On the other hand, in the reversed stack geometry shown in Fig. 5.13b, a high-frequency mode is observed for front pumping at $\sim 135 \mathrm{GHz}$. This frequency corresponds to the first-order resonant mode of a 20.8-nm W-layer, the first one in the stack. In contrast, this mode is not excited when pumping the layer from the substrate side, which means that the excitation has been acoustically decoupled by the thin layers from the rest of the sample.

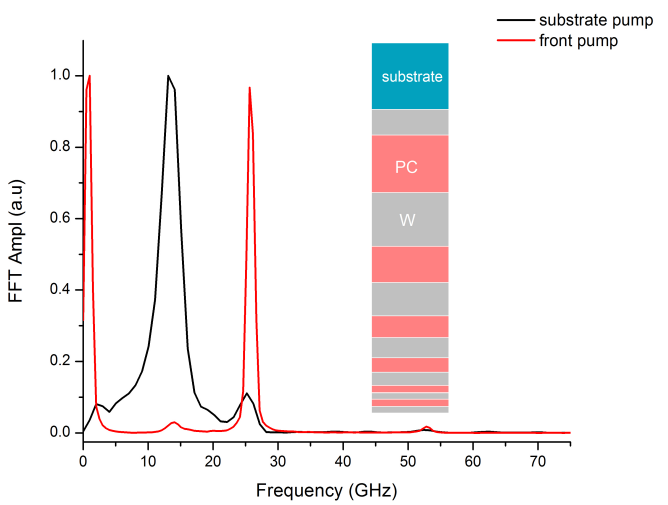

(a)

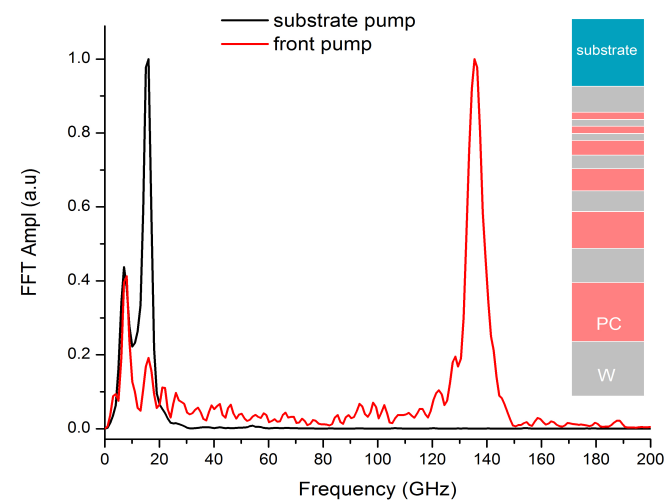

(b)

Figure 5.13: Normalized FFT amplitude of correlated reflectivity dynamics signal after incoherent background subtraction on a W/PC multilayer for front and substrate pumping geometries. The data shown in a) corresponds to the sample: $2.5 \mathrm{~nm} \mathrm{W/} 2.7 \mathrm{nmPC} / 2.5 \mathrm{~nm} \mathrm{~W} / 2.7 \mathrm{~nm} \mathrm{PC/} 5.0 \mathrm{~nm} \mathrm{~W} / 5.5 \mathrm{~nm} \mathrm{PC/}$ $7.6 \mathrm{~nm} \mathrm{W/} 8.2 \mathrm{~nm} \mathrm{PC/} 12.6 \mathrm{~nm} \mathrm{W/} 13.7 \mathrm{~nm} \mathrm{PC/} 20.2 \mathrm{~nm} \mathrm{W/} 21.8 \mathrm{~nm} \mathrm{PC/9.6}$ $\mathrm{nm} \mathrm{W}$ on glass substrate. The data shown in b)corresponds to the sample with a reversed stacking as shown in the schematics.

\subsection{Discussion}

In this chapter some of the most salient features of the elastic dynamics in different structures have been presented. Optically generated strain pulses can excite broadband, rich dynamics in multilayer systems. Additionally, optical detection allows to characterize the elastic response and quantify important material parameters, such as sound velocity even in transparent films, interface reflec- 
tion coefficient and excitation bandwidth. These parameters can be used in numerical simulations, and as an additional evidence of film high quality.

In periodic multilayer systems bulk modes in the acoustic band and highfrequency surface modes in the band gap have been detected in a reasonable agreement with theory. The wavelength of the surface mode is determined by the multilayer periodicity, so that in a system with only few stack repetitions the blocking of this mode cannot be efficiently achieved. However, when the multilayer periodicity is broken by varying the layer thickness in an aperiodic manner, a more efficient amplitude suppression of the high-frequency mode is observed, in the same total film thickness.

In acoustically matched system, the bulk mode persists even in the aperiodic multilayer. On the other hand in systems with high acoustic mismatch also the excitation of low-frequency bulk-like modes is hindered. 


\section{Chapter 6}

\section{Conclusion and outlook}

In this work we have investigated magnetization and elastic dynamics in nanopatterned thin films and multilayers.

Magnetization dynamics on antidot lattices shows an emerging complex behavior when increasing the aspect ratio between the film thickness and distance between antidots. In particular, spin-waves with a frequency below, as well as above the uniform precession mode are detected with a very weak dependence on external magnetic field. The bulk spin-wave modes such as uniform precession and perpendicular standing spin wave mode emerge at increasingly higher magnetic fields. This points to the development of large areas with inhomogeneous internal field, where high-frequency localized modes can be optically excited.

In magnonic crystals with a low aspect ratio, additional features were determined such as an increase in spin-wave damping and a lower uniform precession frequency due to the reduced internal magnetic field, compared to a non-patterned film.

Furthermore, we exploited the advantages of the all-optical experimental setup, namely by performing spatially-resolved measurements and making use of a high-intensity beam that allows to create a high-temperature spatial profile. In these experiments, we determined that the temperature profile locally quenches the saturation magnetization, which leads to a spin-wave frequency shift along the pump-beam profile. Any spin wave traveling away from the excitation spot would propagate towards an increasing effective field. This means that the spin wave frequency needs to be up-converted, in order to adapt to the local spin-wave frequency, i.e. the spin waves would be continuously reflected back at the boundary with cooler region. This further hinders spin-wave propagation in a magnonic crystal.

In addition to a rich magnetization dynamics, in antidot lattices with large aspect ratio we detect elastic dynamics with characteristic frequencies in giga- 
hertz range, that depend on intrinsic antidot lattice parameters such as the distance between antidots or their size. This highlights the favorable feature of our experimental setup that allows the recording of both elastic and magnetization dynamics in the same measurement. Moreover, photoexcited elastic modes in gigahertz regime might greatly influence magnetization dynamics characteristics through the magneto-elastic coupling, and must be taken into account when dealing with magnonic crystals. In this context, we could take further advantage of distinctive characteristics of the experimental setup in order to gain a better understanding of magnetization dynamics. For example, spatiallyresolved elastic dynamics measurements could clarify the spatial propagation characteristics of the excited acoustic waves in $\mathrm{GHz}$ regime. On the other hand, the magneto-optical response of a material depends on its particular band structure, so that the Kerr rotation and ellipticity might have a different linear response to changes in lattice parameters due to strain ${ }^{92}$. Thus, when dealing with strain-induced magnetization dynamics it might be more convenient to measure ellipticity.

Elastic dynamics in multilayers indicate that both bulk (in the acoustic band) and surface modes (in the band gap) can be excited. By breaking the periodicity in the acoustically matched $\mathrm{CoFeB} / \mathrm{MgO}$ multilayer system, the amplitude of the high-frequency surface mode can be efficiently reduced. In addition, in the acoustically mismatched W/PC multilayers the excitation of some bulk-mode frequencies is hindered.

Furthermore, nano-scale $\mathrm{CoFeB} / \mathrm{MgO}$ multilayers can be thought of as a magnonic superlattices ${ }^{93}$, i.e., a one-dimensional magnonic crystal. As we have seen, the excited surface-mode frequency can be tailored by tuning the band gap frequency, that depends on lattice periodicity. Thus, such a multilayer could be used as a model system to study the interplay between elastic modes and magnetization oscillations. 


\section{Bibliography}

[1] B. Lenk, H. Ulrichs, F. Garbs, and M. Münzenberg, Physics Reports 507, 107 (2011).

[2] V. V. Kruglyak, S. O. Demokritov, and D. Grundler, Journal of Physics D: Applied Physics 43, 264001 (2010).

[3] A. A. Serga, A. V. Chumak, and B. Hillebrands, Journal of Physics D: Applied Physics 43, 264002 (2010).

[4] R. L. Stamps, S. Breitkreutz, J. Akerman, A. V. Chumak, Y. Otani, G. E. W. Bauer, J.-U. Thiele, M. Bowen, S. A. Majetich, M. Kläui, et al., Journal of Physics D: Applied Physics 47, 333001 (2014).

[5] M. Maldovan, Nature 503, 209 (2013).

[6] R. M. Costescu, D. G. Cahill, F. H. Fabreguette, Z. A. Sechrist, and S. M. George, Science (New York, N.Y.) 303, 989 (2004).

[7] C. Chiritescu, D. G. Cahill, N. Nguyen, D. Johnson, A. Bodapati, P. Keblinski, and P. Zschack, Science (New York, N.Y.) 315, 351 (2007).

[8] P. Norris, N. Q. Le, and C. H. Baker, Journal of Heat Transfer 135, 061604 (2013).

[9] D. Nardi, M. Travagliati, M. E. Siemens, Q. Li, M. M. Murnane, H. C. Kapteyn, G. Ferrini, F. Parmigiani, and F. Banfi, Nano letters 11, 4126 (2011).

[10] T. Gorishnyy, C. K. Ullal, M. Maldovan, G. Fytas, and E. L. Thomas, Phys. Rev. Lett. 94, 115501 (2005).

[11] H. Ulrichs, B. Lenk, and M. Münzenberg, Applied Physics Letters 97, 092506 (2010). 
[12] M. Vogel, A. V. Chumak, E. H. Waller, T. Langner, V. I. Vasyuchka, B. Hillebrands, and G. von Freymann, Nature physics letters 11, 487 (2015).

[13] A. G. Gurevich and G. A. Melkov, Magnetization Oscillations and Waves (CRC Press, Boca Raton, 1996).

[14] J. Stöhr and H. C. Siegmann, Magnetism: From fundamentals to nanoscale dynamics, vol. 152 (Springer, Berlin, 2006).

[15] M. G. Cottam, Linear and nonlinear spin waves in magnetic films and superlattices (World Scientific, Singapore and River Edge, NJ, 1994).

[16] D. D. Stancil, Theory of Magnetostatic Waves (Springer New York, New York, NY, 1993).

[17] W. Heisenberg, Zeitschrift für Physik 69, 287 (1931).

[18] T. Arai, Phys. Rev. 126, 471 (1962).

[19] B. D. Cullity and C. D. Graham, Introduction to magnetic materials (IEEE/Wiley, Hoboken, N.J., 2009), 2nd ed.

[20] G. V. Swamy, R. K. Rakshit, R. P. Pant, and G. A. Basheed, Journal of Applied Physics 117, 17A312 (2015).

[21] A. T. Hindmarch, A. W. Rushforth, R. P. Campion, C. H. Marrows, and B. L. Gallagher, Phys. Rev. B 83, 212404 (2011).

[22] M. G. Cottam and D. J. Lockwood, Light scattering in magnetic solids (Wiley, New York and Chichester, 1986).

[23] L. Landau and L. Evgeni, Phys. Zeitsch. der Sow. 8, 153169 (1935).

[24] T. Gilbert, Physical Review 100, 1235 (1955).

[25] T. Holstein and H. Primakoff, Phys. Rev. 58, 1098 (1940).

[26] C. Kittel, Phys. Rev. 73, 155 (1948).

[27] R. Damon and J. Eshbach, J.Phys. Chem. Solids 19, 308 (1961).

[28] M. Hurben and C. Patton, Journal of Magnetism and Magnetic Materials 139, 263 (1995).

[29] M. Krawczyk and D. Grundler, Journal of physics: Condensed matter 26, $123202(2014)$ 
[30] B. Lenk, N. Abeling, J. Panke, and M. Münzenberg, Journal of Applied Physics 112, 083921 (2012).

[31] R. Mandal, P. Laha, K. Das, S. Saha, S. Barman, A. K. Raychaudhuri, and A. Barman, Applied Physics Letters 103, 262410 (2013).

[32] S. O. Demokritov and A. N. Slavin, Magnonics: From fundamentals to applications, vol. 125 of Topics in applied physics (Springer, Berlin and New York, 2013).

[33] S. Neusser, B. Botters, M. Becherer, D. Schmitt-Landsiedel, and D. Grundler, Applied Physics Letters 93, 122501 (2008).

[34] M. Kostylev, G. Gubbiotti, G. Carlotti, G. Socino, S. Tacchi, C. Wang, N. Singh, A. O. Adeyeye, and R. L. Stamps, Journal of Applied Physics 103, 07C507 (2008).

[35] S. Neusser, G. Duerr, S. Tacchi, M. Madami, M. L. Sokolovskyy, G. Gubbioti, M. Krawczyk, and D. Grundler, Phys. Rev. B 84, 094454 (2011).

[36] K. Y. Guslienko, S. O. Demokritov, B. Hillebrands, and A. N. Slavin, Phys. Rev. B 66, 132402 (2002).

[37] S. Tacchi, P. Gruszecki, M. Madami, G. Carlotti, J. W. Kłos, M. Krawczyk, A. Adeyeye, and G. Gubbiotti, Scientific reports 5, 10367 (2015).

[38] H. Puszkarski, M. Krawczyk, and H. Diep, Surface Science 602, 2197 (2008).

[39] M. Bailleul, D. Olligs, C. Fermon, and S. O. Demokritov, Europhysics Letters 56, 741 (2001).

[40] M. Bailleul, R. Höllinger, and C. Fermon, Phys. Rev. B 73, 104424 (2006).

[41] D. Xing, H. Yang, and Y. Cao, Journal of Magnetism and Magnetic Materials 377, 286 (2015).

[42] J. W. Kłos, D. Kumar, M. Krawczyk, and A. Barman, Scientific reports 3, 2444 (2013).

[43] J. W. Kos, M. Krawczyk, and M. Sokolovskyy, Journal of Applied Physics 109, 07D311 (2011).

[44] G. Chen, Nanoscale energy transport and conversion: A parallel treatment of electrons, molecules, phonons, and photons, MIT-Pappalardo series in mechanical engineering (Oxford University Press, New York, 2005). 
[45] L. Landau and E. Lifshitz, Course of Theoretical Physics: Volume 7, Theory of Elasticity (Pergamon Press, 1959).

[46] L. M. Brekhovskikh, Waves in Layered Media (Elsevier Science, Burlington, 1960).

[47] P. A. Deymier, Acoustic metamaterials and phononic crystals, vol. 173 of Springer series in solid-state sciences (Springer, Berlin and New York, 2013).

[48] A. M. Kosevich, The crystal lattice: Phonons, solitons, dislocations, superlattices (Wiley-VCH, Berlin and New York, 2005), 2nd ed.

[49] S. Rytov, Soviet Phys. Acoustics 2, 67 (1956).

[50] E. Beaurepaire, J.-C. Merle, A. Daunois, and J.-Y. Bigot, Phys. Rev. Lett. 76, 4250 (1996).

[51] C. Thomsen, H. T. Grahn, H. J. Maris, and J. Tauc, Phys. Rev. B 34, 4129 (1986).

[52] W. S. Capinski, H. J. Maris, T. Ruf, M. Cardona, K. Ploog, and D. S. Katzer, Phys. Rev. B 59, 8105 (1999).

[53] O. Matsuda, M. C. Larciprete, R. L. Voti, and O. B. Wright, Ultrasonics 56, 3 (2015).

[54] O. B. Wright, Journal of Applied Physics 71, 1617 (1992).

[55] O. B. Wright and T. Hyoguchi, Optics Letters 16, 1529 (1991).

[56] E. Elboudouti, Surface Science Reports 64, 471 (2009).

[57] G. A. Antonelli, H. J. Maris, S. G. Malhotra, and J. M. E. Harper, Journal of Applied Physics 91, 3261 (2002).

[58] C. Thomsen, H. T. Grahn, H. J. Maris, and J. Tauc, Le Journal de Physique Colloques 46, C10 (1985).

[59] O. Matsuda and O. B. Wright, J. Opt. Soc. Am. B 19, 3028 (2002).

[60] H.-N. Lin, R. J. Stoner, H. J. Maris, and J. Tauc, Journal of Applied Physics 69, 3816 (1991).

[61] M. van Kampen, C. Jozsa, J. T. Kohlhepp, P. LeClair, L. Lagae, W. J. M. de Jonge, and B. Koopmans, Phys. Rev. Lett. 88, 227201 (2002). 
[62] M. Vomir, L. H. F. Andrade, L. Guidoni, E. Beaurepaire, and J.-Y. Bigot, Physical review letters 94, 237601 (2005).

[63] M. Djordjevic and M. Münzenberg, Phys. Rev. B 75, 012404 (2007).

[64] B. Lenk, G. Eilers, J. Hamrle, and M. Münzenberg, Phys. Rev. B 82, 134443 (2010).

[65] F. Busse, M. Mansurova, B. Lenk, M. von der Ehe, and M. Münzenberg, Scientific Reports 5, 12824 (2015).

[66] M. Born and E. Wolf, Principles of Optics (Cambridge University Press, 1999), 7th ed.

[67] J. Kerr, Philosophical Magazine Series 5 3, 321 (1877).

[68] R. P. Hunt, Journal of Applied Physics 38, 1652 (1967).

[69] Z. J. Yang and M. R. Scheinfein, Journal of Applied Physics 74, 6810 (1993).

[70] A. Zvezdin and V. Kotov, Modern Magnetooptics and Magnetooptical Materials: Studies in Condensed Matter (Taylor, 1997).

[71] C. You and S. Shin, Applied Physics Letters 69, 1315 (1996).

[72] X. Liang, X. Xu, R. Zheng, Z. A. Lum, and J. Qiu, Appl. Opt. 54, 1557 (2015).

[73] B. Koopmans, M. van Kampen, J. T. Kohlhepp, and W. J. M. de Jonge, Phys. Rev. Lett. 85, 844 (2000).

[74] G. M. G. J. Crangle, Proceedings of the Royal Society of London. Series A, Mathematical and Physical Sciences 321, 477 (1971).

[75] P. Hansen, P. Rschmann, and W. Tolksdorf, Journal of Applied Physics 45, 2728 (1974).

[76] V. Zbarsky, Ph.D. thesis, Georg-August-Universitat (2015).

[77] B. Lenk, Ph.D. thesis, Georg-August-Universitat, Goettingen (2013).

[78] F. Company, xT Nova NanoLab User's Manual (2007).

[79] H.-U. Krebs and O. Bremert, Applied Physics Letters 62, 2341 (1993).

[80] F. Döring, C. Eberl, S. Schlenkrich, F. Schlenkrich, S. Hoffmann, T. Liese, H. Krebs, S. Pisana, T. Santos, H. Schuhmann, et al., Applied Physics A 119, 11 (2015). 
[81] J. Röder, T. Liese, and H. Krebs, Journal of Applied Physics 107, 103515 (2010).

[82] A. Vansteenkiste, J. Leliaert, M. Dvornik, M. Helsen, F. Garcia-Sanchez, and B. Van Waeyenberge, AIP Advances 4, 107133 (2014).

[83] M. Djordjevic, Ph.D. thesis, Georg-August-Universitat (2010).

[84] G. M. Müller, G. Eilers, Z. Wang, M. Scherff, R. Ji, K. Nielsch, C. A. Ross, and M. Münzenberg, New Journal of Physics 10, 123004 (2008).

[85] A. Kamra, H. Keshtgar, P. Yan, and G. E. W. Bauer, Physical Review B 91 (2015).

[86] O. Kolokoltsev, N. Qureshi, E. Mejia-Uriarte, and C. L. Ordoñez Romero, Journal of Applied Physics 112, 013902 (2012).

[87] G. V. Swamy, H. Pandey, A. K. Srivastava, M. K. Dalai, K. K. Maurya, Rashmi, and R. K. Rakshit, AIP Advances 3, 072129 (2013).

[88] K. Norimatsu, J. Hu, A. Goto, K. Igarashi, T. Sasagawa, and K. G. Nakamura, Solid State Communications 157, 58 (2013).

[89] B.-T. Wang and P. Zhang, Applied Physics Letters 100, 082109 (2012).

[90] F. Boschini, M. Mansurova, G. Mussler, J. Kampmeier, D. Grützmacher, L. Braun, F. Katmis, J. S. Moodera, C. Dallera, E. Carpene, et al., Scientific Reports 5, 15304 (2015).

[91] A. Selfridge, IEEE Transactions on Sonics and Ultrasonics 32, 381 (1985).

[92] J.-W. Kim, M. Vomir, and J.-Y. Bigot, Physical review letters 109, 166601 (2012).

[93] S. A. Nikitov, P. Tailhades, and C. S. Tsai, Journal of Magnetism and Magnetic Materials 236, 320 (2001). 


\section{Curriculum Vitae}

\section{Personal Data}

Name Maria Mansurova

Date of birth 10.07.1986

Place of birth Moscow, Russia

Nationality Mexican

Address Weender Landstraße 54, 37075, Göttingen

\section{Education}

10.2001-05.2004 American School of Puebla, Puebla, (Mexico)

08.2004-06.2009 Bachelor of Physics, Faculty of Sciences, National Autonomous University of México, Distrito Federal (México)

08.2009 - 10.2011 Master of Sciences (Physics), Faculty of Sciences, National Autonomous University of México, Distrito Federal (México)

06.2012 - PhD student in the group of Prof. Dr. Markus Münzenberg, I.Physikalisches Institut, Georg-August-Universität, Göttingen

Göttingen, 01.2016 